North Dakota Meteorological Conditions Resulting in Top 25 Concentrations 
This supplemental document presents meteorological conditions resulting in peak hourly $\mathrm{SO}_{2}$ observed and predicted concentration at the five North Dakota monitors (shown in the figure below).

- Table 1-5 show the meteorological conditions for the North Dakota peak observed concentrations using beta $u^{*}$ AERMET.

- Table 6-10 show the meteorological conditions for the North Dakota peak predicted concentrations using default AERMET and default AERMOD (Test Case 1).

- Table $11-15$ show the meteorological conditions for the North Dakota peak predicted concentrations using beta $u^{*}$ AERMET and default AERMOD (Test Case 2).

- Table 16-20 show the meteorological conditions for the North Dakota peak predicted concentrations with beta $u^{*}$ AERMET and beta AERMOD with sigma-v=0.5 m/s options. (Test Case 4)

- Table 21 - 25 show the meteorological conditions for the North Dakota peak predicted concentrations with beta $u^{*}$ AERMET and With SHARP (Test Case 5).

In the tables below, the respective columns indicate ranked concentrations, year-month-day-hour, friction velocity $\left(\mathrm{u}_{*}\right.$, $\mathrm{m} / \mathrm{s})$, convective velocity scale $\left(\mathrm{w}_{*}, \mathrm{~m} / \mathrm{s}\right)$, vertical potential temperature gradient at the top of the mixing lid (available if a convective, daytime hour - VPTG in deg $\mathrm{C} / \mathrm{m}$ ), convective mixing height (Zic in $\mathrm{m}$ ), mechanical mixing height (Zim in $\mathrm{m}$ ), Monin-Obukhov length ( $\mathrm{L}$, in $\mathrm{m}$ ), surface roughness length ( $\mathrm{zO}$, in $\mathrm{m}$ ), 10-m wind speed (WSPD, in $\mathrm{m} / \mathrm{s}), 10-\mathrm{m}$ wind direction (WDIR, in deg), and the surface air temperature in deg $\mathrm{K}$.

Figure 1 Map of North Dakota Model Evaluation Layout

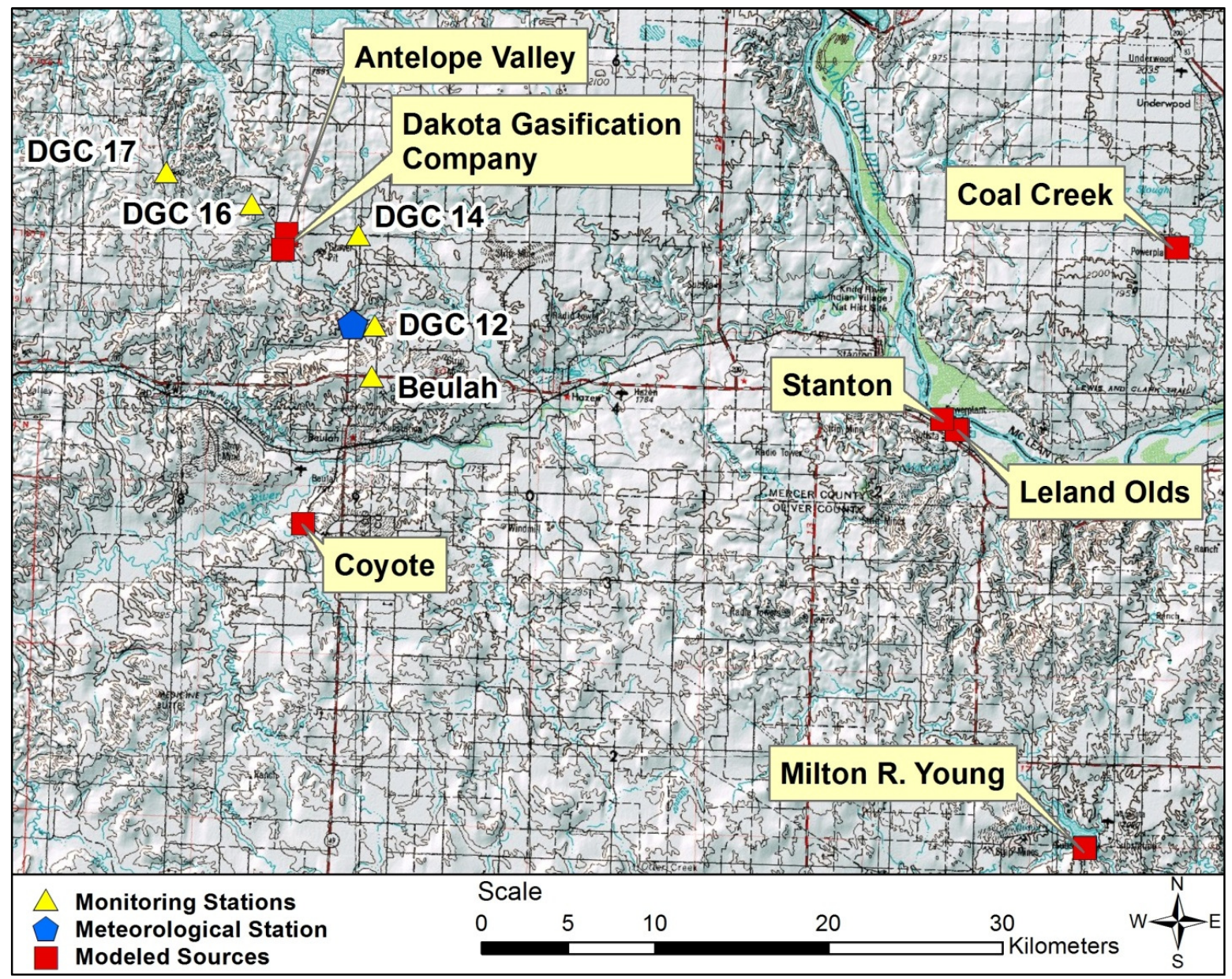


Table 1 Meteorological Conditions for Top 25 Observed Concentrations at North Dakota DGC \#12 Monitor Using Beta $u^{*}$ AERMET

\begin{tabular}{|c|c|c|c|c|c|c|c|c|c|c|c|c|}
\hline \multicolumn{2}{|c|}{ Rank Conc. } & YYMMDDHH & $\mathrm{u}^{*}$ & $\mathbf{w}^{*}$ & VPTG & $Z_{i c}$ & $z_{\text {im }}$ & $\mathbf{L}$ & $z_{0}$ & WSPD & WDIR & temp \\
\hline 1 & 432.28 & 10030222 & 0.048 & -9 & -9 & -999 & 25 & 9.2 & 0.007 & 0.89 & 144.2 & 265.6 \\
\hline 2 & 199.11 & 10120624 & 0.095 & -9 & -9 & -999 & 70 & 12.1 & 0.013 & 1.76 & 286 & 256.9 \\
\hline 3 & 157.19 & 7031617 & 0.222 & 0.38 & 0.016 & 359 & 252 & -179.2 & 0.007 & 3.93 & 100.6 & 274.6 \\
\hline 4 & 154.57 & 7092709 & 0.671 & 0.601 & 0.005 & 186 & 1318 & -649.8 & 0.105 & 7.55 & 316.5 & 285.5 \\
\hline 5 & 149.33 & 10042110 & 0.276 & 0.822 & 0.008 & 216 & 349 & -20.6 & 0.047 & 3.17 & 102.5 & 287.5 \\
\hline 6 & 136.23 & 9091612 & 0.255 & 1.424 & 0.005 & 565 & 309 & -8.1 & 0.105 & 2.15 & 246.7 & 301.5 \\
\hline 7 & 120.51 & 10030604 & 0.104 & -9 & -9 & -999 & 81 & 19.1 & 0.007 & 2.06 & 2 & 269.7 \\
\hline 8 & 117.89 & 9090212 & 0.305 & 1.57 & 0.005 & 674 & 404 & -12.3 & 0.105 & 2.73 & 91.4 & 297.5 \\
\hline 9 & 110.03 & 8110213 & 0.378 & 0.635 & 0.005 & 128 & 557 & -67.3 & 0.014 & 5.85 & 95.2 & 289.5 \\
\hline 10 & 110.03 & 9082410 & 0.723 & 0.932 & 0.008 & 291 & 1472 & -340.1 & 0.105 & 8.05 & 311.5 & 293.7 \\
\hline 11 & 104.79 & 8070408 & 0.518 & 0.703 & 0.005 & 180 & 893 & -179.7 & 0.101 & 5.72 & 188.9 & 296.4 \\
\hline 12 & 104.79 & 10122421 & 0.133 & -9 & -9 & -999 & 117 & 26.5 & 0.007 & 2.59 & 157.2 & 263.9 \\
\hline 13 & 99.55 & 9080911 & 0.393 & 2.009 & 0.007 & 1060 & 591 & -19.8 & 0.105 & 3.71 & 12.6 & 292.4 \\
\hline 14 & 99.55 & 10081809 & 0.419 & 0.834 & 0.012 & 247 & 651 & -78.5 & 0.105 & 4.42 & 75.3 & 290 \\
\hline 15 & 99.55 & 10120807 & 0.06 & -9 & -9 & -999 & 35 & 10.4 & 0.007 & 0.85 & 140.2 & 258.4 \\
\hline 16 & 96.93 & 7071712 & 0.446 & 1.318 & 0.015 & 559 & 714 & -54 & 0.105 & 4.6 & 97.2 & 299.7 \\
\hline 17 & 96.93 & 7083010 & 0.403 & 0.997 & 0.005 & 294 & 614 & -48.5 & 0.101 & 4.16 & 199.9 & 298.9 \\
\hline 18 & 91.70 & 7081416 & 0.536 & 1.541 & 0.005 & 818 & 943 & -85.8 & 0.105 & 5.68 & 98.6 & 292.8 \\
\hline 19 & 91.70 & 7091910 & 0.564 & 0.441 & 0.01 & 126 & 1016 & -662.3 & 0.105 & 6.35 & 105.4 & 288 \\
\hline 20 & 91.70 & 8031910 & 0.19 & 0.05 & 0.012 & 9 & 202 & -1291.3 & 0.007 & 3.44 & 93 & 276 \\
\hline 21 & 86.46 & 7061209 & 0.322 & 1.209 & 0.014 & 641 & 470 & -30.3 & 0.105 & 3.17 & 121.3 & 296.7 \\
\hline 22 & 86.46 & 10100511 & 0.369 & 1.283 & 0.02 & 432 & 538 & -25.7 & 0.105 & 3.58 & 326 & 294.5 \\
\hline 23 & 83.84 & 9091012 & 0.333 & 1.402 & 0.005 & 510 & 461 & -17.1 & 0.101 & 3.13 & 206.6 & 302.4 \\
\hline 24 & 81.22 & 9111711 & 0.318 & 0.641 & 0.013 & 147 & 430 & -44.8 & 0.014 & 4.83 & 203.7 & 284.9 \\
\hline 25 & 81.22 & 10030522 & 0.155 & -9 & -9 & -999 & 146 & 36.7 & 0.007 & 2.99 & 97.4 & 269.8 \\
\hline
\end{tabular}

Table 2 Meteorological Conditions for Top 25 Observed Concentrations at North Dakota DGC \#14 Monitor Using Beta $u^{\star}$ AERMET

\begin{tabular}{|c|c|c|c|c|c|c|c|c|c|c|c|c|}
\hline \multicolumn{2}{|c|}{ Rank Conc. } & \multirow{2}{*}{\begin{tabular}{|r|} 
YYMMDDHH \\
10120701 \\
\end{tabular}} & \multirow{2}{*}{$\begin{array}{c}\mathrm{u}^{*} \\
0.092 \\
\end{array}$} & \multirow{2}{*}{$\begin{array}{c}\mathbf{w}^{*} \\
-9 \\
\end{array}$} & \multirow{2}{*}{$\begin{array}{c}\text { VPTG } \\
-9 \\
\end{array}$} & \multirow{2}{*}{$\begin{array}{l}Z_{\text {ic }} \\
-999 \\
\end{array}$} & \multirow{2}{*}{$\begin{array}{l}z_{\text {im }} \\
67 \\
\end{array}$} & \multirow{2}{*}{$\frac{\mathbf{L}}{11.9}$} & \multirow{2}{*}{$\begin{array}{c}\mathrm{z}_{0} \\
0.011 \\
\end{array}$} & \multirow{2}{*}{$\begin{array}{c}\text { WSPD } \\
1.76 \\
\end{array}$} & \multirow{2}{*}{$\begin{array}{l}\text { WDIR } \\
316 \\
\end{array}$} & \multirow{2}{*}{$\begin{array}{l}\text { temp } \\
256.8 \\
\end{array}$} \\
\hline 1 & 259.37 & & & & & & & & & & & \\
\hline 2 & 196.49 & 10020113 & 0.075 & 0.109 & 0.021 & 115 & 49 & -93 & 0.007 & 1.3 & 177.1 & 258.4 \\
\hline 3 & 183.39 & 10030223 & 0.043 & -9 & -9 & -999 & 21 & 9.8 & 0.007 & 0.67 & 148.9 & 265.5 \\
\hline 4 & 170.29 & 10033114 & 0.341 & 0.808 & 0.005 & 322 & 479 & -60.7 & 0.007 & 5.85 & 273 & 286.5 \\
\hline 5 & 162.43 & 7121109 & 0.322 & -9 & -9 & -999 & 440 & 157.1 & 0.007 & 6.03 & 294.8 & 267.8 \\
\hline 6 & 141.47 & 10030603 & 0.092 & -9 & -9 & -999 & 67 & 16.6 & 0.007 & 1.83 & 2.4 & 269.6 \\
\hline 7 & 120.51 & 7031618 & 0.193 & -9 & -9 & -999 & 203 & 80.5 & 0.007 & 3.66 & 114.1 & 274.1 \\
\hline 8 & 115.27 & 7091512 & 0.212 & 1.266 & 0.009 & 401 & 235 & -4.7 & 0.105 & 1.65 & 312.5 & 293.6 \\
\hline 9 & 112.65 & 10042109 & 0.259 & 0.627 & 0.008 & 149 & 317 & -26.4 & 0.047 & 3.04 & 88.8 & 286 \\
\hline 10 & 104.79 & 10030523 & 0.124 & -9 & -9 & -999 & 104 & 23.3 & 0.007 & 2.41 & 73 & 269.7 \\
\hline 11 & 99.55 & 10053109 & 0.358 & 0.836 & 0.005 & 242 & 515 & -47.8 & 0.047 & 4.38 & 260.4 & 290.4 \\
\hline 12 & 94.31 & 7042409 & 0.179 & 0.433 & 0.006 & 91 & 183 & -16.3 & 0.042 & 2.06 & 219 & 287.9 \\
\hline 13 & 91.70 & 10100610 & 0.364 & 1.011 & 0.019 & 332 & 527 & -38.7 & 0.105 & 3.66 & 253.7 & 287.8 \\
\hline 14 & 89.08 & 7081418 & 0.443 & 1.256 & 0.005 & 951 & 712 & -104 & 0.105 & 4.74 & 86.2 & 291.3 \\
\hline 15 & 89.08 & 9062908 & 0.615 & 0.58 & 0.008 & 145 & 1156 & -434 & 0.105 & 6.88 & 89.4 & 291.9 \\
\hline 16 & 86.46 & 10080909 & 0.398 & 0.961 & 0.005 & 314 & 602 & -55.5 & 0.105 & 4.11 & 248.4 & 299 \\
\hline 17 & 86.46 & 10082011 & 0.588 & -9 & -9 & -999 & 1081 & -106.3 & 0.105 & 6.3 & 295.1 & 303.8 \\
\hline 18 & 83.84 & 7102714 & 0.207 & 1.162 & 0.005 & 552 & 227 & -7.8 & 0.105 & 1.74 & 235.3 & 278.1 \\
\hline 19 & 83.84 & 10120807 & 0.06 & -9 & -9 & -999 & 35 & 10.4 & 0.007 & 0.85 & 140.2 & 258.4 \\
\hline 20 & 81.22 & 7042809 & 0.555 & 0.662 & 0.005 & 153 & 992 & -226.1 & 0.047 & 7.24 & 256.3 & 293.8 \\
\hline 21 & 81.22 & 9111513 & 0.214 & 0.984 & 0.008 & 308 & 239 & -7.9 & 0.014 & 2.86 & 243.7 & 279.4 \\
\hline 22 & 78.60 & 10122421 & 0.133 & -9 & -9 & -999 & 117 & 26.5 & 0.007 & 2.59 & 157.2 & 263.9 \\
\hline 23 & 75.98 & 7021112 & 0.207 & 0.36 & 0.007 & 116 & 226 & -54.8 & 0.007 & 3.53 & 86.3 & 263.9 \\
\hline 24 & 75.98 & 7051310 & 0.331 & 0.853 & 0.009 & 255 & 457 & -37.2 & 0.047 & 3.98 & 62.1 & 296 \\
\hline 25 & 75.98 & 8110214 & 0.349 & 0.653 & 0.005 & 150 & 495 & -57.1 & 0.014 & 5.36 & 95.7 & 289.9 \\
\hline
\end{tabular}


Table 3 Meteorological Conditions for Top 25 Observed Concentrations at North Dakota DGC \#16 Monitor Using Beta $u^{\star}$ AERMET

\begin{tabular}{|c|c|c|c|c|c|c|c|c|c|c|c|c|}
\hline \multicolumn{2}{|c|}{ Rank Conc. } & \multirow{2}{*}{\begin{tabular}{|r} 
YYMMDDHH \\
10030223
\end{tabular}} & \multirow{2}{*}{$\frac{u^{*}}{0.043}$} & \multirow{2}{*}{$\begin{array}{c}\mathbf{w}^{*} \\
-9 \\
\end{array}$} & \multirow{2}{*}{\begin{tabular}{|l} 
VPTG \\
-9 \\
\end{tabular}} & \multirow{2}{*}{$\begin{array}{c}Z_{\text {ic }} \\
-999\end{array}$} & \multirow{2}{*}{$\frac{z_{\text {im }}}{21}$} & \multirow{2}{*}{$\frac{\mathbf{L}}{9.8}$} & \multirow{2}{*}{$\begin{array}{c}z_{0} \\
0.007\end{array}$} & \multirow{2}{*}{$\begin{array}{c}\text { WSPD } \\
0.67 \\
\end{array}$} & \multirow{2}{*}{$\frac{\text { WDIR }}{148.9}$} & \multirow{2}{*}{$\frac{\text { temp }}{265.5}$} \\
\hline 1 & 374.64 & & & & & & & & & & & \\
\hline 2 & 151.95 & 9061417 & 0.381 & 1.523 & 0.008 & 2467 & 575 & -96.8 & 0.105 & 4.07 & 130 & 295.6 \\
\hline 3 & 125.75 & 7031618 & 0.193 & -9 & -9 & -999 & 203 & 80.5 & 0.007 & 3.66 & 114.1 & 274.1 \\
\hline 4 & 123.13 & 10120808 & 0.065 & -9 & -9 & -999 & 39 & 9.3 & 0.007 & 1.12 & 157.5 & 258.3 \\
\hline 5 & 115.27 & 10030523 & 0.124 & -9 & -9 & -999 & 104 & 23.3 & 0.007 & 2.41 & 73 & 269.7 \\
\hline 6 & 115.27 & 10110713 & 0.098 & 0.818 & 0.019 & 205 & 74 & -1 & 0.014 & 0.98 & 240.8 & 289.6 \\
\hline 7 & 107.41 & 8020316 & 0.177 & -9 & -9 & -999 & 179 & 96.3 & 0.007 & 3.35 & 86.6 & 264.4 \\
\hline 8 & 104.79 & 10030605 & 0.136 & -9 & -9 & -999 & 120 & 28.3 & 0.007 & 2.64 & 5.5 & 269.6 \\
\hline 9 & 104.79 & 10051510 & 0.33 & 0.927 & 0.005 & 257 & 454 & -28.9 & 0.047 & 3.89 & 149.8 & 294.1 \\
\hline 10 & 99.55 & 10020914 & 0.32 & 0.49 & 0.01 & 216 & 434 & -150.6 & 0.01 & 5.36 & 146 & 255.4 \\
\hline 11 & 89.08 & 7120507 & 0.133 & -9 & -9 & -999 & 117 & 25.6 & 0.007 & 2.59 & 99.9 & 255.5 \\
\hline 12 & 86.46 & 7081418 & 0.443 & 1.256 & 0.005 & 951 & 712 & -104 & 0.105 & 4.74 & 86.2 & 291.3 \\
\hline 13 & 86.46 & 10081112 & 0.319 & 1.753 & 0.005 & 955 & 433 & -14.4 & 0.105 & 2.91 & 109 & 305 \\
\hline 14 & 81.22 & 10122204 & 0.209 & -9 & -9 & -999 & 230 & 31.9 & 0.01 & 3.86 & 112 & 257 \\
\hline 15 & 78.60 & 7072102 & 0.867 & -9 & -9 & -999 & 1937 & 834.2 & 0.105 & 9.88 & 148 & 293.4 \\
\hline 16 & 78.60 & 8082111 & 0.407 & 1.436 & 0.013 & 626 & 623 & -35.6 & 0.105 & 4.07 & 105 & 302.8 \\
\hline 17 & 78.60 & 8110213 & 0.378 & 0.635 & 0.005 & 128 & 557 & -67.3 & 0.014 & 5.85 & 95.2 & 289.5 \\
\hline 18 & 78.60 & 9062908 & 0.615 & 0.58 & 0.008 & 145 & 1156 & -434 & 0.105 & 6.88 & 89.4 & 291.9 \\
\hline 19 & 78.60 & 10081212 & 0.283 & 1.561 & 0.005 & 682 & 361 & -10.1 & 0.105 & 2.46 & 49.7 & 301.2 \\
\hline 20 & 75.98 & 7021113 & 0.24 & 0.45 & 0.006 & 161 & 282 & -60.8 & 0.007 & 4.11 & 66.4 & 263.9 \\
\hline 21 & 75.98 & 7032004 & 0.242 & -9 & -9 & -999 & 287 & 44.9 & 0.007 & 4.65 & 130.2 & 270.5 \\
\hline 22 & 75.98 & 7042917 & 0.257 & 1.354 & 0.005 & 1047 & 313 & -17.9 & 0.047 & 2.91 & 102.8 & 293.9 \\
\hline 23 & 75.98 & 7050221 & -9 & -9 & -9 & -999 & -999 & -99999 & 0.047 & 11.58 & 147.2 & 289.1 \\
\hline 24 & 75.98 & 8071010 & 0.517 & 1.215 & 0.005 & 397 & 896 & -76.6 & 0.105 & 5.45 & 127.1 & 301.3 \\
\hline 25 & 75.98 & 10030301 & 0.043 & -9 & -9 & -999 & 21 & 9.8 & 0.007 & 0.67 & 178 & 265.4 \\
\hline
\end{tabular}

Table 4 Meteorological Conditions for Top 25 Observed Concentrations at North Dakota DGC \#17 Monitor Using Beta $\mathbf{u}^{\star}$ AERMET

\begin{tabular}{|c|c|c|c|c|c|c|c|c|c|c|c|c|}
\hline \multicolumn{2}{|c|}{ Rank Conc. } & \multirow{2}{*}{\begin{tabular}{|r} 
YYMMDDHH \\
10030223 \\
\end{tabular}} & \multirow{2}{*}{$\begin{array}{c}\mathrm{u}^{*} \\
0.043 \\
\end{array}$} & \multirow{2}{*}{$\begin{array}{l}\mathbf{w}^{*} \\
-9 \\
\end{array}$} & \multirow{2}{*}{$\begin{array}{c}\text { VPTG } \\
-9 \\
\end{array}$} & \multirow{2}{*}{$\frac{Z_{\text {ic }}}{-999}$} & \multirow{2}{*}{$\frac{z_{\text {im }}}{21}$} & \multirow{2}{*}{$\frac{\mathbf{L}}{9.8}$} & \multirow{2}{*}{$\begin{array}{c}\mathrm{z}_{0} \\
0.007 \\
\end{array}$} & \multirow{2}{*}{$\begin{array}{c}\text { WSPD } \\
0.67 \\
\end{array}$} & \multirow{2}{*}{$\begin{array}{l}\text { WDIR } \\
148.9 \\
\end{array}$} & \multirow{2}{*}{$\frac{\text { temp }}{265.5}$} \\
\hline 1 & 306.52 & & & & & & & & & & & \\
\hline 2 & 154.57 & \begin{tabular}{|l|}
10072109 \\
\end{tabular} & 0.374 & 0.89 & 0.005 & 234 & 549 & -43.4 & 0.105 & 3.8 & 99.5 & 294.1 \\
\hline 3 & 115.27 & 7120508 & 0.211 & -9 & -9 & -999 & 232 & 64.3 & 0.007 & 4.02 & 104.7 & 255.8 \\
\hline 4 & 112.65 & 8071009 & 0.565 & 0.914 & 0.005 & 233 & 1020 & -137 & 0.105 & 6.12 & 120.6 & 299.1 \\
\hline 5 & 110.03 & 7031618 & 0.193 & -9 & -9 & -999 & 203 & 80.5 & 0.007 & 3.66 & 114.1 & 274.1 \\
\hline 6 & 104.79 & 7090410 & -9 & -9 & -9 & -999 & -999 & -99999 & 0.105 & 7.11 & 105.1 & 299.8 \\
\hline 7 & 104.79 & 8110213 & 0.378 & 0.635 & 0.005 & 128 & 557 & -67.3 & 0.014 & 5.85 & 95.2 & 289.5 \\
\hline 8 & 104.79 & 10030524 & 0.077 & -9 & -9 & -999 & 52 & 13.8 & 0.007 & 1.56 & 96.1 & 269.4 \\
\hline 9 & 99.55 & 7021113 & 0.24 & 0.45 & 0.006 & 161 & 282 & -60.8 & 0.007 & 4.11 & 66.4 & 263.9 \\
\hline 10 & 96.93 & 10030601 & 0.06 & -9 & -9 & -999 & 35 & 10.6 & 0.007 & 1.21 & 224.2 & 269.2 \\
\hline 11 & 94.31 & 7081408 & 0.32 & 0.641 & 0.016 & 226 & 434 & -70 & 0.105 & 3.35 & 104.5 & 290.6 \\
\hline 12 & 89.08 & 10061409 & 0.277 & 0.887 & 0.005 & 209 & 349 & -15.8 & 0.105 & 2.55 & 117.3 & 291.9 \\
\hline 13 & 86.46 & 10120808 & 0.065 & -9 & -9 & -999 & 39 & 9.3 & 0.007 & 1.12 & 157.5 & 258.3 \\
\hline 14 & 83.84 & 8020316 & 0.177 & -9 & -9 & -999 & 179 & 96.3 & 0.007 & 3.35 & 86.6 & 264.4 \\
\hline 15 & 81.22 & 7061101 & 0.081 & -9 & -9 & -999 & 56 & 14.1 & 0.105 & 0.94 & 345 & 290.3 \\
\hline 16 & 81.22 & 7061210 & 0.235 & 1.078 & 0.014 & 687 & 278 & -17.7 & 0.105 & 2.19 & 105.4 & 296.8 \\
\hline 17 & 81.22 & 7081810 & 0.4 & 0.606 & 0.013 & 258 & 615 & -185.5 & 0.105 & 4.38 & 97.4 & 290 \\
\hline 18 & 81.22 & 9062508 & 0.315 & 0.519 & 0.009 & 100 & 425 & -56.2 & 0.105 & 3.26 & 126.3 & 295.8 \\
\hline 19 & 78.60 & 7051203 & 0.153 & -9 & -9 & -999 & 143 & 23 & 0.047 & 2.23 & 93.4 & 278.7 \\
\hline 20 & 78.60 & 8052712 & 0.327 & 1.582 & 0.008 & 929 & 449 & -20.5 & 0.047 & 3.75 & 96.1 & 283.7 \\
\hline 21 & 78.60 & 8071503 & 0.155 & -9 & -9 & -999 & 147 & 25.4 & 0.105 & 1.92 & 258.8 & 287.5 \\
\hline 22 & 78.60 & 9071109 & 0.378 & 1.065 & 0.012 & 388 & 558 & -43.4 & 0.105 & 3.84 & 120.1 & 290 \\
\hline 23 & 78.60 & 10122204 & 0.209 & -9 & -9 & -999 & 230 & 31.9 & 0.01 & 3.86 & 112 & 257 \\
\hline 24 & 75.98 & 9081010 & 0.259 & 1.571 & 0.005 & 645 & 316 & -7.2 & 0.105 & 2.15 & 252.1 & 296.5 \\
\hline 25 & 75.98 & 7043002 & 0.259 & -9 & -9 & -999 & 329 & 61.9 & 0.047 & 3.66 & 59.3 & 284.4 \\
\hline
\end{tabular}


Table 5 Meteorological Conditions for Top 25 Observed Concentrations at North Dakota Beulah Monitor Using Beta $u^{*}$ AERMET

\begin{tabular}{|c|c|c|c|c|c|c|c|c|c|c|c|c|}
\hline \multicolumn{2}{|c|}{ Rank Conc. } & \multirow{2}{*}{\begin{tabular}{|r|} 
YYMMDDHH \\
10030222 \\
\end{tabular}} & \multirow{2}{*}{$\frac{u^{*}}{0.048}$} & \multirow{2}{*}{$\begin{array}{c}\mathbf{w}^{*} \\
-9 \\
\end{array}$} & \multirow{2}{*}{$\begin{array}{c}\text { VPTG } \\
-9 \\
\end{array}$} & \multirow{2}{*}{$\begin{array}{c}Z_{\text {ic }} \\
-999\end{array}$} & \multirow{2}{*}{$\frac{z_{\text {im }}}{25}$} & \multirow{2}{*}{$\frac{\mathbf{L}}{9.2}$} & \multirow{2}{*}{$\frac{\mathbf{z}_{0}}{0.007}$} & \multirow{2}{*}{$\frac{\text { WSPD }}{0.89}$} & \multirow{2}{*}{$\frac{\text { WDIR }}{144.2}$} & \multirow{2}{*}{$\begin{array}{l}\text { temp } \\
265.6 \\
\end{array}$} \\
\hline 1 & 429.66 & & & & & & & & & & & \\
\hline 2 & 172.91 & 8052708 & 0.323 & 0.586 & 0.005 & 153 & 442 & -64.4 & 0.047 & 4.02 & 88.8 & 279.8 \\
\hline 3 & 146.71 & 10042110 & 0.276 & 0.822 & 0.008 & 216 & 349 & -20.6 & 0.047 & 3.17 & 102.5 & 287.5 \\
\hline 4 & 141.47 & 7071710 & 0.504 & 1.085 & 0.006 & 427 & 859 & -107.3 & 0.105 & 5.41 & 93.5 & 297.3 \\
\hline 5 & 138.85 & 10100512 & 0.395 & 1.418 & 0.016 & 484 & 596 & -26.1 & 0.105 & 3.84 & 319.3 & 295.9 \\
\hline 6 & 136.23 & 10033014 & 0.343 & 0.65 & 0.005 & 177 & 482 & -65.2 & 0.007 & 5.9 & 340.3 & 289.9 \\
\hline 7 & 136.23 & 10072214 & 0.378 & 1.424 & 0.009 & 949 & 557 & -44.2 & 0.105 & 3.84 & 341.3 & 295.8 \\
\hline 8 & 133.61 & 7031616 & 0.278 & 0.661 & 0.016 & 356 & 351 & -66.2 & 0.007 & 4.78 & 98.6 & 275.6 \\
\hline 9 & 117.89 & 10042009 & 0.439 & 0.561 & 0.005 & 108 & 698 & -129.4 & 0.047 & 5.63 & 96.7 & 289.5 \\
\hline 10 & 115.27 & 10120808 & 0.065 & -9 & -9 & -999 & 39 & 9.3 & 0.007 & 1.12 & 157.5 & 258.3 \\
\hline 11 & 110.03 & 7042709 & 0.378 & 0.749 & 0.007 & 231 & 562 & -74.7 & 0.047 & 4.74 & 321.9 & 285.9 \\
\hline 12 & 107.41 & 9082311 & 0.808 & 1.411 & 0.005 & 389 & 1738 & -182.7 & 0.105 & 8.85 & 100.5 & 301.2 \\
\hline 13 & 104.79 & 7083009 & 0.439 & 0.731 & 0.005 & 190 & 699 & -102.6 & 0.101 & 4.74 & 204.3 & 296.3 \\
\hline 14 & 99.55 & 9090212 & 0.305 & 1.57 & 0.005 & 674 & 404 & -12.3 & 0.105 & 2.73 & 91.4 & 297.5 \\
\hline 15 & 99.55 & 10030605 & 0.136 & -9 & -9 & -999 & 120 & 28.3 & 0.007 & 2.64 & 5.5 & 269.6 \\
\hline 16 & 91.70 & 7101311 & 0.333 & 0.36 & 0.01 & 72 & 469 & -143.3 & 0.105 & 3.62 & 89.6 & 283.8 \\
\hline 17 & 91.70 & 8080510 & 0.377 & 1.044 & 0.005 & 290 & 554 & -33.9 & 0.105 & 3.75 & 338.6 & 296.6 \\
\hline 18 & 83.84 & 8073009 & 0.427 & 0.792 & 0.005 & 170 & 670 & -66.8 & 0.105 & 4.47 & 105 & 299.6 \\
\hline 19 & 83.84 & 10122422 & 0.116 & -9 & -9 & -999 & 95 & 21.2 & 0.007 & 2.28 & 165.6 & 263.9 \\
\hline 20 & 81.22 & 8020319 & 0.177 & -9 & -9 & -999 & 179 & 47.1 & 0.007 & 3.4 & 63.2 & 264.8 \\
\hline 21 & 81.22 & 10081809 & 0.419 & 0.834 & 0.012 & 247 & 651 & -78.5 & 0.105 & 4.42 & 75.3 & 290 \\
\hline 22 & 78.60 & 9062609 & 0.358 & 0.861 & 0.008 & 262 & 515 & -47.1 & 0.105 & 3.66 & 53.9 & 297.6 \\
\hline 23 & 78.60 & 10120701 & 0.092 & -9 & -9 & -999 & 67 & 11.9 & 0.011 & 1.76 & 316 & 256.8 \\
\hline 24 & 73.36 & 7090409 & -9 & -9 & -9 & -999 & -999 & -99999 & 0.105 & 6.75 & 100.9 & 296.6 \\
\hline 25 & 73.36 & 7092212 & 0.412 & 1.236 & 0.007 & 385 & 635 & -35.6 & 0.101 & 4.16 & 184 & 300.5 \\
\hline
\end{tabular}

Table 6 Meteorological Conditions for Top 25 Modeled Concentrations at North Dakota DGC \#12 Monitor Using Default AERMET and Default AERMOD

\begin{tabular}{|c|c|c|c|c|c|c|c|c|c|c|c|c|}
\hline Rank & Conc. & YYMMDDHH & $u^{*}$ & $\mathbf{w}^{*}$ & VPTG & $Z_{i c}$ & $z_{i m}$ & $\mathbf{L}$ & $z_{0}$ & WSPD & WDIR & temp \\
\hline 1 & 248.99 & 7021312 & 0.05 & 0.218 & 0.016 & 262 & 27 & -8.1 & 0.007 & 0.76 & 103 & 251.5 \\
\hline 2 & 218.99 & 10020916 & 0.107 & 0.267 & 0.012 & 223 & 84 & -36.2 & 0.007 & 1.79 & 100.5 & 255.4 \\
\hline 3 & 161.75 & 10022714 & 0.174 & 0.335 & 0.027 & 307 & 174 & -107.1 & 0.007 & 3.04 & 100.9 & 263.1 \\
\hline 4 & 160.06 & 8070608 & 0.239 & 0.608 & 0.005 & 115 & 281 & -17.5 & 0.105 & 2.23 & 310.5 & 299.3 \\
\hline 5 & 146.17 & 9022211 & 0.17 & 0.207 & 0.017 & 30 & 168 & -41.4 & 0.007 & 2.86 & 99.6 & 259.3 \\
\hline 6 & 143.69 & 10110616 & 0.07 & 0.608 & 0.016 & 273 & 45 & -1.1 & 0.014 & 0.72 & 318.6 & 290.4 \\
\hline 7 & 127.11 & 8020314 & 0.173 & 0.494 & 0.024 & 277 & 172 & -29.6 & 0.007 & 2.86 & 97.1 & 265 \\
\hline 8 & 111.85 & 8122514 & 0.21 & 0.076 & 0.013 & 17 & 232 & -874.4 & 0.007 & 3.8 & 100 & 260.1 \\
\hline 9 & 111.81 & 7031617 & 0.222 & 0.38 & 0.016 & 359 & 252 & -179.2 & 0.007 & 3.93 & 100.6 & 274.6 \\
\hline 10 & 111.77 & 7081308 & 0.228 & 0.576 & 0.021 & 177 & 262 & -27.6 & 0.105 & 2.23 & 104.4 & 293.8 \\
\hline 11 & 106.16 & 8022212 & 0.204 & 0.36 & 0.018 & 76 & 221 & -34.2 & 0.007 & 3.4 & 96.7 & 272.5 \\
\hline 12 & 104.83 & 9021613 & 0.148 & 0.252 & 0.03 & 226 & 136 & -114.6 & 0.007 & 2.59 & 96.3 & 262.8 \\
\hline 13 & 104.59 & 8032014 & 0.25 & 0.429 & 0.013 & 345 & 300 & -171.1 & 0.007 & 4.42 & 100.3 & 273 \\
\hline 14 & 102.69 & 8081508 & 0.177 & 0.471 & 0.011 & 107 & 179 & -14.1 & 0.105 & 1.61 & 97.9 & 293.5 \\
\hline 15 & 100.21 & 7052018 & 0.289 & 0.491 & 0.026 & 382 & 372 & -194.4 & 0.047 & 3.75 & 100.6 & 287.8 \\
\hline 16 & 98.00 & 10110716 & 0.06 & 0.588 & 0.013 & 270 & 35 & -1 & 0.014 & 0.58 & 330 & 287.4 \\
\hline 17 & 97.84 & 8020214 & 0.158 & 0.102 & 0.026 & 146 & 151 & -1372 & 0.007 & 2.86 & 99.5 & 266 \\
\hline 18 & 96.44 & 9020413 & 0.143 & 0.426 & 0.031 & 173 & 131 & -16.3 & 0.007 & 2.28 & 195.8 & 271.7 \\
\hline 19 & 95.46 & 9112915 & 0.095 & 0.334 & 0.007 & 252 & 91 & -14.5 & 0.014 & 1.34 & 320 & 275.1 \\
\hline 20 & 95.28 & 9090208 & 0.23 & 0.553 & 0.013 & 301 & 264 & -54.1 & 0.105 & 2.37 & 102.8 & 292.6 \\
\hline 21 & 91.52 & 8121713 & 0.186 & 0.11 & 0.015 & 18 & 193 & -216.8 & 0.007 & 3.31 & 98.5 & 254.6 \\
\hline 22 & 90.67 & 9022414 & 0.204 & 0.588 & 0.035 & 248 & 222 & -25.8 & 0.007 & 3.35 & 200.4 & 267.2 \\
\hline 23 & 89.02 & 8030915 & 0.128 & 0.709 & 0.005 & 387 & 111 & -5.8 & 0.007 & 1.88 & 317.3 & 276.8 \\
\hline 24 & 88.05 & 10042110 & 0.276 & 0.822 & 0.008 & 216 & 349 & -20.6 & 0.047 & 3.17 & 102.5 & 287.5 \\
\hline 25 & 87.44 & 8051809 & 0.262 & 0.841 & 0.012 & 257 & 322 & -19.5 & 0.047 & 2.99 & 97 & 292.2 \\
\hline
\end{tabular}


Table 7 Meteorological Conditions for Top 25 Modeled Concentrations at North Dakota DGC \#14 Monitor Using Default AERMET and Default AERMOD

\begin{tabular}{|c|c|c|c|c|c|c|c|c|c|c|c|c|}
\hline Rank & Conc. & YYMMDDHH & $u^{*}$ & $\mathbf{w}^{*}$ & VPTG & $Z_{i c}$ & $z_{i m}$ & $\mathbf{L}$ & $z_{0}$ & WSPD & WDIR & temp \\
\hline 1 & 237.77 & 10030515 & 0.104 & 0.33 & 0.038 & 322 & 80 & -25 & 0.007 & 1.7 & 109.5 & 270.6 \\
\hline 2 & 228.04 & 7030611 & 0.075 & 0.525 & 0.047 & 267 & 50 & -2 & 0.007 & 0.98 & 262.2 & 272.1 \\
\hline 3 & 198.22 & 10032717 & 0.188 & 0.527 & 0.009 & 348 & 199 & -39.8 & 0.007 & 3.17 & 262.9 & 274.1 \\
\hline 4 & 190.92 & 8071609 & 0.246 & 0.894 & 0.012 & 226 & 293 & -11.8 & 0.105 & 2.19 & 257.5 & 298.6 \\
\hline 5 & 172.02 & 7021312 & 0.05 & 0.218 & 0.016 & 262 & 27 & -8.1 & 0.007 & 0.76 & 103 & 251.5 \\
\hline 6 & 165.60 & 10031316 & 0.16 & 0.206 & 0.018 & 117 & 154 & -139.2 & 0.007 & 2.82 & 108.8 & 274.3 \\
\hline 7 & 161.29 & 9020812 & 0.19 & 0.234 & 0.027 & 36 & 198 & -48.3 & 0.007 & 3.22 & 107.9 & 271.6 \\
\hline 8 & 131.95 & 10040317 & 0.434 & 1.382 & 0.005 & 1486 & 686 & -115 & 0.047 & 5.54 & 258 & 282.1 \\
\hline 9 & 117.93 & 7081308 & 0.228 & 0.576 & 0.021 & 177 & 262 & -27.6 & 0.105 & 2.23 & 104.4 & 293.8 \\
\hline 10 & 117.14 & 9022212 & 0.196 & 0.338 & 0.018 & 64 & 209 & -31.3 & 0.007 & 3.26 & 109.2 & 260.5 \\
\hline 11 & 114.84 & 8112514 & 0.197 & 0.634 & 0.025 & 226 & 215 & -17 & 0.014 & 2.82 & 263.2 & 279.3 \\
\hline 12 & 108.52 & 8020313 & 0.171 & 0.467 & 0.024 & 235 & 169 & -28.7 & 0.007 & 2.82 & 104.2 & 264.9 \\
\hline 13 & 107.80 & 8031111 & 0.174 & 0.411 & 0.009 & 83 & 175 & -15.7 & 0.007 & 2.77 & 110.7 & 283.2 \\
\hline 14 & 105.56 & 10042209 & 0.229 & 0.561 & 0.011 & 103 & 263 & -17.6 & 0.047 & 2.59 & 105.8 & 288.8 \\
\hline 15 & 102.71 & 8071408 & 0.249 & 0.734 & 0.006 & 216 & 299 & -21.2 & 0.105 & 2.37 & 267.5 & 298.9 \\
\hline 16 & 100.30 & 7052811 & 0.202 & 0.767 & 0.013 & 301 & 218 & -13.8 & 0.047 & 2.23 & 265.1 & 288.7 \\
\hline 17 & 97.07 & 9020313 & 0.161 & 0.135 & 0.02 & 186 & 158 & -797.8 & 0.007 & 2.91 & 191.5 & 262.3 \\
\hline 18 & 97.06 & 8022316 & 0.22 & 0.342 & 0.009 & 118 & 247 & -78 & 0.007 & 3.8 & 106.8 & 276.9 \\
\hline 19 & 96.89 & 7121713 & 0.162 & 0.109 & 0.012 & 19 & 156 & -156.4 & 0.007 & 2.86 & 104.4 & 274.2 \\
\hline 20 & 94.80 & 7060909 & 0.288 & 1.235 & 0.006 & 356 & 374 & -11.3 & 0.105 & 2.55 & 267.6 & 294.9 \\
\hline 21 & 91.53 & 7091510 & 0.236 & 0.934 & 0.015 & 259 & 275 & -10.4 & 0.105 & 2.06 & 261.8 & 288.9 \\
\hline 22 & 90.58 & 8032015 & 0.249 & 0.402 & 0.013 & 349 & 298 & -207.2 & 0.007 & 4.42 & 109.4 & 273.4 \\
\hline 23 & 87.31 & 9031615 & 0.138 & 0.6 & 0.019 & 184 & 123 & -5.6 & 0.007 & 2.01 & 111.3 & 279.6 \\
\hline 24 & 86.83 & 10033115 & 0.302 & 0.614 & 0.005 & 331 & 399 & -98.5 & 0.007 & 5.27 & 260.4 & 286.6 \\
\hline 25 & 85.23 & 10111214 & 0.213 & 0.899 & 0.006 & 343 & 237 & -11.5 & 0.014 & 2.95 & 272.2 & 281.3 \\
\hline
\end{tabular}

Table 8 Meteorological Conditions for Top 25 Modeled Concentrations at North Dakota DGC \#16 Monitor Using Default AERMET and Default AERMOD

\begin{tabular}{|c|c|c|c|c|c|c|c|c|c|c|c|c|}
\hline Rank & Conc. & YYMMDDHH & $\mathrm{u}^{*}$ & $\mathbf{w}^{*}$ & VPTG & $Z_{i c}$ & $z_{i m}$ & $\mathbf{L}$ & $z_{0}$ & WSPD & WDIR & temp \\
\hline 1 & 201.22 & 10030515 & 0.104 & 0.33 & 0.038 & 322 & 80 & -25 & 0.007 & 1.7 & 109.5 & 270.6 \\
\hline 2 & 191.31 & 10040315 & 0.46 & 1.264 & 0.005 & 1312 & 750 & -158.2 & 0.047 & 5.94 & 150.5 & 282.5 \\
\hline 3 & 145.15 & 7021312 & 0.05 & 0.218 & 0.016 & 262 & 27 & -8.1 & 0.007 & 0.76 & 103 & 251.5 \\
\hline 4 & 144.07 & 7081609 & 0.154 & 0.831 & 0.009 & 239 & 146 & -3.8 & 0.105 & 1.16 & 139.9 & 292.8 \\
\hline 5 & 139.96 & 9020812 & 0.19 & 0.234 & 0.027 & 36 & 198 & -48.3 & 0.007 & 3.22 & 107.9 & 271.6 \\
\hline 6 & 138.90 & 10031316 & 0.16 & 0.206 & 0.018 & 117 & 154 & -139.2 & 0.007 & 2.82 & 108.8 & 274.3 \\
\hline 7 & 133.92 & 7061608 & 0.144 & 0.964 & 0.009 & 269 & 131 & -2.2 & 0.105 & 0.98 & 133 & 290.4 \\
\hline 8 & 128.46 & 10072516 & 0.509 & 2.095 & 0.01 & 1813 & 877 & -65 & 0.105 & 5.32 & 150.4 & 303.2 \\
\hline 9 & 126.43 & 9061708 & 0.272 & 0.821 & 0.01 & 403 & 341 & -36.8 & 0.105 & 2.73 & 141.8 & 289.3 \\
\hline 10 & 126.36 & 8100212 & 0.239 & 1.117 & 0.007 & 327 & 280 & -8 & 0.105 & 2.01 & 127.7 & 295.1 \\
\hline 11 & 120.68 & 10021213 & 0.152 & 0.623 & 0.019 & 329 & 142 & -11.9 & 0.007 & 2.37 & 125 & 264.6 \\
\hline 12 & 114.79 & 9062018 & 0.482 & 1.666 & 0.005 & 2273 & 833 & -137.6 & 0.105 & 5.23 & 132.9 & 298.5 \\
\hline 13 & 113.20 & 9080710 & 0.139 & 0.827 & 0.009 & 380 & 125 & -4.5 & 0.105 & 1.07 & 152.1 & 292.1 \\
\hline 14 & 111.94 & 10072119 & 0.383 & 1.143 & 0.005 & 2003 & 569 & -188.5 & 0.105 & 4.2 & 148.4 & 294 \\
\hline 15 & 104.72 & 7081308 & 0.228 & 0.576 & 0.021 & 177 & 262 & -27.6 & 0.105 & 2.23 & 104.4 & 293.8 \\
\hline 16 & 102.92 & 10062808 & 0.118 & 0.928 & 0.008 & 417 & 98 & -2.2 & 0.105 & 0.8 & 147.5 & 289 \\
\hline 17 & 99.48 & 10080911 & 0.326 & 1.275 & 0.006 & 415 & 447 & -17.4 & 0.105 & 3.04 & 130.8 & 303.5 \\
\hline 18 & 98.88 & 9022212 & 0.196 & 0.338 & 0.018 & 64 & 209 & -31.3 & 0.007 & 3.26 & 109.2 & 260.5 \\
\hline 19 & 97.44 & 10103013 & 0.238 & 1.182 & 0.011 & 397 & 279 & -8.1 & 0.105 & 2.01 & 120.4 & 278.4 \\
\hline 20 & 95.82 & 8020313 & 0.171 & 0.467 & 0.024 & 235 & 169 & -28.7 & 0.007 & 2.82 & 104.2 & 264.9 \\
\hline 21 & 95.21 & 8082602 & 0.811 & -9 & -9 & -999 & 1757 & 678.4 & 0.105 & 9.39 & 150.1 & 292 \\
\hline 22 & 93.70 & 7100411 & 0.157 & 0.979 & 0.005 & 276 & 150 & -2.9 & 0.105 & 1.12 & 138.7 & 286 \\
\hline 23 & 93.39 & 8013115 & 0.36 & 0.364 & 0.02 & 211 & 519 & -513.7 & 0.007 & 6.48 & 153.7 & 272 \\
\hline 24 & 92.33 & 10042209 & 0.229 & 0.561 & 0.011 & 103 & 263 & -17.6 & 0.047 & 2.59 & 105.8 & 288.8 \\
\hline 25 & 90.09 & 9090618 & 0.521 & 1.146 & 0.008 & 1098 & 926 & -258 & 0.105 & 5.77 & 131.8 & 298.5 \\
\hline
\end{tabular}


Table 9 Meteorological Conditions for Top 25 Modeled Concentrations at North Dakota DGC \#17 Monitor Using Default AERMET and Default AERMOD

\begin{tabular}{|c|c|c|c|c|c|c|c|c|c|c|c|c|}
\hline Rank & Conc. & YYMMDDHH & $u^{*}$ & $\mathrm{w}^{*}$ & VPTG & $Z_{i c}$ & $z_{i m}$ & $\mathbf{L}$ & $z_{0}$ & WSPD & WDIR & temp \\
\hline 1 & 541.94 & 8013107 & 0.05 & -9 & -9 & -999 & 27 & 3.5 & 0.007 & 1.83 & 131.4 & 252.7 \\
\hline 2 & 294.74 & 8013022 & 0.124 & -9 & -9 & -999 & 106 & 11 & 0.007 & 3.66 & 127.6 & 252.7 \\
\hline 3 & 293.06 & 10121207 & 0.053 & -9 & -9 & -999 & 29 & 3.7 & 0.007 & 1.92 & 108.4 & 249.4 \\
\hline 4 & 288.39 & 7012501 & 0.059 & -9 & -9 & -999 & 35 & 4.3 & 0.007 & 2.15 & 108.7 & 269.3 \\
\hline 5 & 242.86 & 10110819 & 0.071 & -9 & -9 & -999 & 47 & 5.5 & 0.014 & 2.32 & 107.2 & 281.4 \\
\hline 6 & 242.40 & 10030515 & 0.104 & 0.33 & 0.038 & 322 & 80 & -25 & 0.007 & 1.7 & 109.5 & 270.6 \\
\hline 7 & 207.09 & 9062824 & 0.065 & -9 & -9 & -999 & 39 & 6.1 & 0.105 & 1.47 & 107.6 & 287.3 \\
\hline 8 & 197.90 & 8042806 & 0.065 & -9 & -9 & -999 & 40 & 5.5 & 0.047 & 1.74 & 108.3 & 271.4 \\
\hline 9 & 197.88 & 9030707 & 0.049 & -9 & -9 & -999 & 26 & 3.7 & 0.007 & 1.79 & 109.1 & 255.1 \\
\hline 10 & 192.01 & 7022405 & 0.054 & -9 & -9 & -999 & 33 & 5.6 & 0.007 & 1.97 & 107.7 & 271.5 \\
\hline 11 & 190.13 & 9110507 & 0.096 & -9 & -9 & -999 & 72 & 7.4 & 0.014 & 3.17 & 106.9 & 271 \\
\hline 12 & 185.49 & 9083005 & 0.083 & -9 & -9 & -999 & 57 & 7.7 & 0.105 & 1.88 & 109.1 & 280.9 \\
\hline 13 & 183.58 & 8020215 & 0.156 & 0.427 & 0.029 & 200 & 147 & -24.2 & 0.007 & 2.55 & 124.9 & 266.9 \\
\hline 14 & 181.25 & 9112604 & 0.037 & -9 & -9 & -999 & 17 & 2.8 & 0.014 & 1.21 & 109.1 & 265.9 \\
\hline 15 & 179.60 & 8012723 & 0.064 & -9 & -9 & -999 & 39 & 4.7 & 0.007 & 2.32 & 109.4 & 272.1 \\
\hline 16 & 178.90 & 10011719 & 0.031 & -9 & -9 & -999 & 13 & 2.2 & 0.007 & 1.12 & 109.7 & 266.8 \\
\hline 17 & 177.51 & 10121919 & 0.04 & -9 & -9 & -999 & 20 & 2.9 & 0.007 & 1.47 & 106.2 & 256.6 \\
\hline 18 & 177.45 & 10021212 & 0.107 & 0.513 & 0.019 & 232 & 85 & -5.3 & 0.007 & 1.56 & 121.1 & 263.8 \\
\hline 19 & 177.13 & 9031504 & 0.031 & -9 & -9 & -999 & 13 & 2.2 & 0.007 & 1.12 & 106.9 & 269.9 \\
\hline 20 & 173.14 & 8010907 & 0.063 & -9 & -9 & -999 & 38 & 4.5 & 0.007 & 2.28 & 109.3 & 264.3 \\
\hline 21 & 170.84 & 8122205 & 0.066 & -9 & -9 & -999 & 41 & 4.6 & 0.007 & 2.41 & 109.1 & 244.8 \\
\hline 22 & 166.71 & 8010319 & 0.061 & -9 & -9 & -999 & 51 & 4.5 & 0.007 & 2.23 & 109.3 & 269.8 \\
\hline 23 & 163.95 & 8021502 & 0.086 & -9 & -9 & -999 & 61 & 6.1 & 0.007 & 3.13 & 108 & 257.7 \\
\hline 24 & 158.68 & 9032017 & 0.136 & 0.466 & 0.023 & 307 & 120 & -19 & 0.007 & 2.19 & 113.8 & 279.8 \\
\hline 25 & 158.47 & 10102108 & 0.088 & -9 & -9 & -999 & 63 & 8.3 & 0.105 & 2.01 & 108.2 & 277.6 \\
\hline
\end{tabular}

Table 10 Meteorological Conditions for Top 25 Modeled Concentrations at North Dakota Beulah Monitor Using Default AERMET and Default AERMOD

\begin{tabular}{|c|c|c|c|c|c|c|c|c|c|c|c|c|}
\hline Rank & Conc. & YYMMDDHH & $u^{*}$ & $\mathbf{w}^{*}$ & VPTG & $Z_{i c}$ & $z_{i m}$ & $\mathbf{L}$ & $z_{0}$ & WSPD & WDIR & temp \\
\hline 1 & 189.96 & 8020312 & 0.15 & 0.359 & 0.023 & 171 & 140 & -31.5 & 0.007 & 2.5 & 95.7 & 264.7 \\
\hline 2 & 184.16 & 7120314 & 0.19 & 0.224 & 0.032 & 41 & 199 & -63.1 & 0.007 & 3.26 & 95.1 & 272.6 \\
\hline 3 & 172.95 & 9021612 & 0.153 & 0.191 & 0.03 & 225 & 143 & -287.2 & 0.007 & 2.73 & 95.5 & 261.5 \\
\hline 4 & 158.73 & 8070609 & 0.287 & 0.889 & 0.005 & 210 & 369 & -17.7 & 0.105 & 2.68 & 332.7 & 300.4 \\
\hline 5 & 148.72 & 8021114 & 0.08 & 0.27 & 0.018 & 351 & 54 & -22.7 & 0.007 & 1.3 & 330.7 & 257.8 \\
\hline 6 & 133.47 & 8022212 & 0.204 & 0.36 & 0.018 & 76 & 221 & -34.2 & 0.007 & 3.4 & 96.7 & 272.5 \\
\hline 7 & 126.90 & 10033013 & 0.304 & 0.634 & 0.005 & 162 & 402 & -44.7 & 0.007 & 5.14 & 323.2 & 289.8 \\
\hline 8 & 121.41 & 10021516 & 0.138 & 0.32 & 0.03 & 183 & 124 & -37.3 & 0.007 & 2.32 & 324.9 & 261.4 \\
\hline 9 & 115.60 & 8031910 & 0.19 & 0.05 & 0.012 & 9 & 202 & -1291.3 & 0.007 & 3.44 & 93 & 276 \\
\hline 10 & 114.08 & 10022712 & 0.193 & 0.575 & 0.026 & 227 & 203 & -21.4 & 0.007 & 3.13 & 98.6 & 262.1 \\
\hline 11 & 113.31 & 9031216 & 0.132 & 0.493 & 0.02 & 192 & 115 & -9.2 & 0.007 & 2.01 & 209 & 264 \\
\hline 12 & 112.57 & 10110716 & 0.06 & 0.588 & 0.013 & 270 & 35 & -1 & 0.014 & 0.58 & 330 & 287.4 \\
\hline 13 & 112.43 & 10042208 & 0.184 & 0.309 & 0.012 & 46 & 190 & -24.7 & 0.047 & 2.15 & 91.1 & 286.1 \\
\hline 14 & 108.50 & 8121714 & 0.16 & 0.087 & 0.016 & 21 & 153 & -328.4 & 0.007 & 2.86 & 96.7 & 255.5 \\
\hline 15 & 108.22 & 10110616 & 0.07 & 0.608 & 0.016 & 273 & 45 & -1.1 & 0.014 & 0.72 & 318.6 & 290.4 \\
\hline 16 & 105.02 & 7031615 & 0.272 & 0.72 & 0.016 & 325 & 341 & -43.9 & 0.007 & 4.6 & 94.5 & 275.8 \\
\hline 17 & 104.78 & 10032315 & 0.146 & 0.393 & 0.012 & 311 & 134 & -40.1 & 0.007 & 2.46 & 326.3 & 274.3 \\
\hline 18 & 102.15 & 7021312 & 0.05 & 0.218 & 0.016 & 262 & 27 & -8.1 & 0.007 & 0.76 & 103 & 251.5 \\
\hline 19 & 101.73 & 8022315 & 0.174 & 0.42 & 0.013 & 112 & 174 & -20.1 & 0.007 & 2.82 & 91.8 & 278.2 \\
\hline 20 & 101.08 & 9020215 & 0.083 & 0.465 & 0.016 & 247 & 58 & -3.6 & 0.007 & 1.16 & 87.2 & 252.9 \\
\hline 21 & 99.13 & 7090309 & 0.15 & 0.786 & 0.025 & 213 & 139 & -3.7 & 0.105 & 1.12 & 333.2 & 298.3 \\
\hline 22 & 99.11 & 8081508 & 0.177 & 0.471 & 0.011 & 107 & 179 & -14.1 & 0.105 & 1.61 & 97.9 & 293.5 \\
\hline 23 & 98.10 & 8031110 & 0.234 & 0.261 & 0.005 & 46 & 273 & -84.3 & 0.007 & 4.07 & 94.8 & 281 \\
\hline 24 & 96.92 & 10051110 & 0.148 & 0.661 & 0.005 & 429 & 137 & -12.1 & 0.047 & 1.61 & 92.3 & 280.6 \\
\hline 25 & 95.51 & 8020213 & 0.131 & 0.101 & 0.026 & 145 & 114 & -798 & 0.007 & 2.37 & 92.9 & 265.1 \\
\hline
\end{tabular}


Table 11 Meteorological Conditions for Top 25 Modeled Concentrations at North Dakota DGC \#12 Monitor Using Beta $u^{\star}$ AERMET and Default AERMOD

\begin{tabular}{|c|c|c|c|c|c|c|c|c|c|c|c|c|}
\hline Rank & Conc. & YYMMDDHH & $u^{*}$ & $\mathrm{w}^{*}$ & VPTG & $Z_{i c}$ & $z_{i m}$ & $\mathbf{L}$ & $z_{0}$ & WSPD & WDIR & temp \\
\hline 1 & 248.99 & 7021312 & 0.05 & 0.218 & 0.016 & 262 & 27 & -8.1 & 0.007 & 0.76 & 103 & 251.5 \\
\hline 2 & 218.99 & 10020916 & 0.107 & 0.267 & 0.012 & 223 & 84 & -36.2 & 0.007 & 1.79 & 100.5 & 255.4 \\
\hline 3 & 161.75 & 10022714 & 0.174 & 0.335 & 0.027 & 307 & 174 & -107.1 & 0.007 & 3.04 & 100.9 & 263.1 \\
\hline 4 & 160.06 & 8070608 & 0.239 & 0.608 & 0.005 & 115 & 281 & -17.5 & 0.105 & 2.23 & 310.5 & 299.3 \\
\hline 5 & 146.17 & 9022211 & 0.17 & 0.207 & 0.017 & 30 & 168 & -41.4 & 0.007 & 2.86 & 99.6 & 259.3 \\
\hline 6 & 143.69 & 10110616 & 0.07 & 0.608 & 0.016 & 273 & 45 & -1.1 & 0.014 & 0.72 & 318.6 & 290.4 \\
\hline 7 & 127.11 & 8020314 & 0.173 & 0.494 & 0.024 & 277 & 172 & -29.6 & 0.007 & 2.86 & 97.1 & 265 \\
\hline 8 & 111.85 & 8122514 & 0.21 & 0.076 & 0.013 & 17 & 232 & -874.4 & 0.007 & 3.8 & 100 & 260.1 \\
\hline 9 & 111.81 & 7031617 & 0.222 & 0.38 & 0.016 & 359 & 252 & -179.2 & 0.007 & 3.93 & 100.6 & 274.6 \\
\hline 10 & 111.77 & 7081308 & 0.228 & 0.576 & 0.021 & 177 & 262 & -27.6 & 0.105 & 2.23 & 104.4 & 293.8 \\
\hline 11 & 106.16 & 8022212 & 0.204 & 0.36 & 0.018 & 76 & 221 & -34.2 & 0.007 & 3.4 & 96.7 & 272.5 \\
\hline 12 & 104.83 & 9021613 & 0.148 & 0.252 & 0.03 & 226 & 136 & -114.6 & 0.007 & 2.59 & 96.3 & 262.8 \\
\hline 13 & 104.59 & 8032014 & 0.25 & 0.429 & 0.013 & 345 & 300 & -171.1 & 0.007 & 4.42 & 100.3 & 273 \\
\hline 14 & 102.69 & 8081508 & 0.177 & 0.471 & 0.011 & 107 & 179 & -14.1 & 0.105 & 1.61 & 97.9 & 293.5 \\
\hline 15 & 100.21 & 7052018 & 0.289 & 0.491 & 0.026 & 382 & 372 & -194.4 & 0.047 & 3.75 & 100.6 & 287.8 \\
\hline 16 & 98.00 & 10110716 & 0.06 & 0.588 & 0.013 & 270 & 35 & -1 & 0.014 & 0.58 & 330 & 287.4 \\
\hline 17 & 97.84 & 8020214 & 0.158 & 0.102 & 0.026 & 146 & 151 & -1372 & 0.007 & 2.86 & 99.5 & 266 \\
\hline 18 & 96.44 & 9020413 & 0.143 & 0.426 & 0.031 & 173 & 131 & -16.3 & 0.007 & 2.28 & 195.8 & 271.7 \\
\hline 19 & 95.46 & 9112915 & 0.095 & 0.334 & 0.007 & 252 & 91 & -14.5 & 0.014 & 1.34 & 320 & 275.1 \\
\hline 20 & 95.28 & 9090208 & 0.23 & 0.553 & 0.013 & 301 & 264 & -54.1 & 0.105 & 2.37 & 102.8 & 292.6 \\
\hline 21 & 91.52 & 8121713 & 0.186 & 0.11 & 0.015 & 18 & 193 & -216.8 & 0.007 & 3.31 & 98.5 & 254.6 \\
\hline 22 & 90.67 & 9022414 & 0.204 & 0.588 & 0.035 & 248 & 222 & -25.8 & 0.007 & 3.35 & 200.4 & 267.2 \\
\hline 23 & 89.02 & 8030915 & 0.128 & 0.709 & 0.005 & 387 & 111 & -5.8 & 0.007 & 1.88 & 317.3 & 276.8 \\
\hline 24 & 88.05 & 10042110 & 0.276 & 0.822 & 0.008 & 216 & 349 & -20.6 & 0.047 & 3.17 & 102.5 & 287.5 \\
\hline 25 & 87.44 & 8051809 & 0.262 & 0.841 & 0.012 & 257 & 322 & -19.5 & 0.047 & 2.99 & 97 & 292.2 \\
\hline
\end{tabular}

Table 12 Meteorological Conditions for Top 25 Modeled Concentrations at North Dakota DGC \#14 Monitor Using Beta $u^{*}$ AERMET and Default AERMOD

\begin{tabular}{|c|c|c|c|c|c|c|c|c|c|c|c|c|}
\hline Rank & Conc. & YYMMDDHH & $\mathrm{u}^{*}$ & $\mathrm{w}^{*}$ & VPTG & $z_{i c}$ & $z_{\text {im }}$ & $\mathbf{L}$ & $\mathrm{z}_{0}$ & WSPD & WDIR & temp \\
\hline 1 & 237.77 & 10030515 & 0.104 & 0.33 & 0.038 & 322 & 80 & -25 & 0.007 & 1.7 & 109.5 & 270.6 \\
\hline 2 & 228.04 & 7030611 & 0.075 & 0.525 & 0.047 & 267 & 50 & -2 & 0.007 & 0.98 & 262.2 & 272.1 \\
\hline 3 & 198.22 & 10032717 & 0.188 & 0.527 & 0.009 & 348 & 199 & -39.8 & 0.007 & 3.17 & 262.9 & 274.1 \\
\hline 4 & 190.92 & 8071609 & 0.246 & 0.894 & 0.012 & 226 & 293 & -11.8 & 0.105 & 2.19 & 257.5 & 298.6 \\
\hline 5 & 172.02 & 7021312 & 0.05 & 0.218 & 0.016 & 262 & 27 & -8.1 & 0.007 & 0.76 & 103 & 251.5 \\
\hline 6 & 165.60 & 10031316 & 0.16 & 0.206 & 0.018 & 117 & 154 & -139.2 & 0.007 & 2.82 & 108.8 & 274.3 \\
\hline 7 & 161.29 & 9020812 & 0.19 & 0.234 & 0.027 & 36 & 198 & -48.3 & 0.007 & 3.22 & 107.9 & 271.6 \\
\hline 8 & 131.95 & 10040317 & 0.434 & 1.382 & 0.005 & 1486 & 686 & -115 & 0.047 & 5.54 & 258 & 282.1 \\
\hline 9 & 117.93 & 7081308 & 0.228 & 0.576 & 0.021 & 177 & 262 & -27.6 & 0.105 & 2.23 & 104.4 & 293.8 \\
\hline 10 & 117.14 & 9022212 & 0.196 & 0.338 & 0.018 & 64 & 209 & -31.3 & 0.007 & 3.26 & 109.2 & 260.5 \\
\hline 11 & 114.84 & 8112514 & 0.197 & 0.634 & 0.025 & 226 & 215 & -17 & 0.014 & 2.82 & 263.2 & 279.3 \\
\hline 12 & 108.52 & 8020313 & 0.171 & 0.467 & 0.024 & 235 & 169 & -28.7 & 0.007 & 2.82 & 104.2 & 264.9 \\
\hline 13 & 107.80 & 8031111 & 0.174 & 0.411 & 0.009 & 83 & 175 & -15.7 & 0.007 & 2.77 & 110.7 & 283.2 \\
\hline 14 & 105.56 & 10042209 & 0.229 & 0.561 & 0.011 & 103 & 263 & -17.6 & 0.047 & 2.59 & 105.8 & 288.8 \\
\hline 15 & 102.71 & 8071408 & 0.249 & 0.734 & 0.006 & 216 & 299 & -21.2 & 0.105 & 2.37 & 267.5 & 298.9 \\
\hline 16 & 100.30 & 7052811 & 0.202 & 0.767 & 0.013 & 301 & 218 & -13.8 & 0.047 & 2.23 & 265.1 & 288.7 \\
\hline 17 & 97.07 & 9020313 & 0.161 & 0.135 & 0.02 & 186 & 158 & -797.8 & 0.007 & 2.91 & 191.5 & 262.3 \\
\hline 18 & 97.06 & 8022316 & 0.22 & 0.342 & 0.009 & 118 & 247 & -78 & 0.007 & 3.8 & 106.8 & 276.9 \\
\hline 19 & 96.89 & 7121713 & 0.162 & 0.109 & 0.012 & 19 & 156 & -156.4 & 0.007 & 2.86 & 104.4 & 274.2 \\
\hline 20 & 94.80 & 7060909 & 0.288 & 1.235 & 0.006 & 356 & 374 & -11.3 & 0.105 & 2.55 & 267.6 & 294.9 \\
\hline 21 & 91.53 & 7091510 & 0.236 & 0.934 & 0.015 & 259 & 275 & -10.4 & 0.105 & 2.06 & 261.8 & 288.9 \\
\hline 22 & 90.58 & 8032015 & 0.249 & 0.402 & 0.013 & 349 & 298 & -207.2 & 0.007 & 4.42 & 109.4 & 273.4 \\
\hline 23 & 87.31 & 9031615 & 0.138 & 0.6 & 0.019 & 184 & 123 & -5.6 & 0.007 & 2.01 & 111.3 & 279.6 \\
\hline 24 & 86.83 & 10033115 & 0.302 & 0.614 & 0.005 & 331 & 399 & -98.5 & 0.007 & 5.27 & 260.4 & 286.6 \\
\hline 25 & 85.23 & 10111214 & 0.213 & 0.899 & 0.006 & 343 & 237 & -11.5 & 0.014 & 2.95 & 272.2 & 281.3 \\
\hline
\end{tabular}


Table 13 Meteorological Conditions for Top 25 Modeled Concentrations at North Dakota DGC \#16 Monitor Using Beta $u^{\star}$ AERMET and Default AERMOD

\begin{tabular}{|c|c|c|c|c|c|c|c|c|c|c|c|c|}
\hline Rank & Conc. & YYMMDDHH & $u^{*}$ & $\mathbf{w}^{*}$ & VPTG & $Z_{i c}$ & $z_{\text {im }}$ & $\mathbf{L}$ & $z_{0}$ & WSPD & WDIR & temp \\
\hline 1 & 201.22 & 10030515 & 0.104 & 0.33 & 0.038 & 322 & 80 & -25 & 0.007 & 1.7 & 109.5 & 270.6 \\
\hline 2 & 191.31 & 10040315 & 0.46 & 1.264 & 0.005 & 1312 & 750 & -158.2 & 0.047 & 5.94 & 150.5 & 282.5 \\
\hline 3 & 145.15 & 7021312 & 0.05 & 0.218 & 0.016 & 262 & 27 & -8.1 & 0.007 & 0.76 & 103 & 251.5 \\
\hline 4 & 144.07 & 7081609 & 0.154 & 0.831 & 0.009 & 239 & 146 & -3.8 & 0.105 & 1.16 & 139.9 & 292.8 \\
\hline 5 & 139.96 & 9020812 & 0.19 & 0.234 & 0.027 & 36 & 198 & -48.3 & 0.007 & 3.22 & 107.9 & 271.6 \\
\hline 6 & 138.90 & 10031316 & 0.16 & 0.206 & 0.018 & 117 & 154 & -139.2 & 0.007 & 2.82 & 108.8 & 274.3 \\
\hline 7 & 133.92 & 7061608 & 0.144 & 0.964 & 0.009 & 269 & 131 & -2.2 & 0.105 & 0.98 & 133 & 290.4 \\
\hline 8 & 128.46 & 10072516 & 0.509 & 2.095 & 0.01 & 1813 & 877 & -65 & 0.105 & 5.32 & 150.4 & 303.2 \\
\hline 9 & 126.43 & 9061708 & 0.272 & 0.821 & 0.01 & 403 & 341 & -36.8 & 0.105 & 2.73 & 141.8 & 289.3 \\
\hline 10 & 126.36 & 8100212 & 0.239 & 1.117 & 0.007 & 327 & 280 & -8 & 0.105 & 2.01 & 127.7 & 295.1 \\
\hline 11 & 120.68 & 10021213 & 0.152 & 0.623 & 0.019 & 329 & 142 & -11.9 & 0.007 & 2.37 & 125 & 264.6 \\
\hline 12 & 114.79 & 9062018 & 0.482 & 1.666 & 0.005 & 2273 & 833 & -137.6 & 0.105 & 5.23 & 132.9 & 298.5 \\
\hline 13 & 113.20 & 9080710 & 0.139 & 0.827 & 0.009 & 380 & 125 & -4.5 & 0.105 & 1.07 & 152.1 & 292.1 \\
\hline 14 & 111.94 & 10072119 & 0.383 & 1.143 & 0.005 & 2003 & 569 & -188.5 & 0.105 & 4.2 & 148.4 & 294 \\
\hline 15 & 104.72 & 7081308 & 0.228 & 0.576 & 0.021 & 177 & 262 & -27.6 & 0.105 & 2.23 & 104.4 & 293.8 \\
\hline 16 & 102.92 & 10062808 & 0.118 & 0.928 & 0.008 & 417 & 98 & -2.2 & 0.105 & 0.8 & 147.5 & 289 \\
\hline 17 & 99.48 & 10080911 & 0.326 & 1.275 & 0.006 & 415 & 447 & -17.4 & 0.105 & 3.04 & 130.8 & 303.5 \\
\hline 18 & 98.88 & 9022212 & 0.196 & 0.338 & 0.018 & 64 & 209 & -31.3 & 0.007 & 3.26 & 109.2 & 260.5 \\
\hline 19 & 97.44 & 10103013 & 0.238 & 1.182 & 0.011 & 397 & 279 & -8.1 & 0.105 & 2.01 & 120.4 & 278.4 \\
\hline 20 & 95.82 & 8020313 & 0.171 & 0.467 & 0.024 & 235 & 169 & -28.7 & 0.007 & 2.82 & 104.2 & 264.9 \\
\hline 21 & 95.42 & 8082602 & 0.811 & -9 & -9 & -999 & 1757 & 678.4 & 0.105 & 9.39 & 150.1 & 292 \\
\hline 22 & 93.70 & 7100411 & 0.157 & 0.979 & 0.005 & 276 & 150 & -2.9 & 0.105 & 1.12 & 138.7 & 286 \\
\hline 23 & 93.39 & 8013115 & 0.36 & 0.364 & 0.02 & 211 & 519 & -513.7 & 0.007 & 6.48 & 153.7 & 272 \\
\hline 24 & 92.33 & 10042209 & 0.229 & 0.561 & 0.011 & 103 & 263 & -17.6 & 0.047 & 2.59 & 105.8 & 288.8 \\
\hline 25 & 90.09 & 9090618 & 0.521 & 1.146 & 0.008 & 1098 & 926 & -258 & 0.105 & 5.77 & 131.8 & 298.5 \\
\hline
\end{tabular}

Table 14 Meteorological Conditions for Top 25 Modeled Concentrations at North Dakota DGC \#17 Monitor Using Beta $u^{*}$ AERMET and Default AERMOD

\begin{tabular}{|c|c|c|c|c|c|c|c|c|c|c|c|c|}
\hline Rank & Conc. & YYMMDDHH & $\mathrm{u}^{*}$ & $\mathrm{w}^{*}$ & VPTG & $Z_{i c}$ & $z_{\text {im }}$ & $\mathbf{L}$ & $z_{0}$ & WSPD & WDIR & temp \\
\hline 1 & 242.40 & 10030515 & 0.104 & 0.33 & 0.038 & 322 & 80 & -25 & 0.007 & 1.7 & 109.5 & 270.6 \\
\hline 2 & 183.58 & 8020215 & 0.156 & 0.427 & 0.029 & 200 & 147 & -24.2 & 0.007 & 2.55 & 124.9 & 266.9 \\
\hline 3 & 177.45 & 10021212 & 0.107 & 0.513 & 0.019 & 232 & 85 & -5.3 & 0.007 & 1.56 & 121.1 & 263.8 \\
\hline 4 & 162.42 & 8013022 & 0.124 & -9 & -9 & -999 & 106 & 11 & 0.007 & 3.66 & 127.6 & 252.7 \\
\hline 5 & 158.68 & 9032017 & 0.136 & 0.466 & 0.023 & 307 & 120 & -19 & 0.007 & 2.19 & 113.8 & 279.8 \\
\hline 6 & 153.94 & 9031615 & 0.138 & 0.6 & 0.019 & 184 & 123 & -5.6 & 0.007 & 2.01 & 111.3 & 279.6 \\
\hline 7 & 149.96 & 7021312 & 0.05 & 0.218 & 0.016 & 262 & 27 & -8.1 & 0.007 & 0.76 & 103 & 251.5 \\
\hline 8 & 149.18 & 8013107 & 0.05 & -9 & -9 & -999 & 27 & 3.5 & 0.007 & 1.83 & 131.4 & 252.7 \\
\hline 9 & 134.46 & 10080810 & 0.292 & 1.03 & 0.005 & 269 & 378 & -15.3 & 0.105 & 2.68 & 125.5 & 304 \\
\hline 10 & 133.98 & 9020812 & 0.19 & 0.234 & 0.027 & 36 & 198 & -48.3 & 0.007 & 3.22 & 107.9 & 271.6 \\
\hline 11 & 131.91 & 10121207 & 0.053 & -9 & -9 & -999 & 29 & 3.7 & 0.007 & 1.92 & 108.4 & 249.4 \\
\hline 12 & 130.76 & 7031613 & 0.192 & 0.533 & 0.018 & 250 & 201 & -29.1 & 0.007 & 3.17 & 115.7 & 275.1 \\
\hline 13 & 125.96 & 10031316 & 0.16 & 0.206 & 0.018 & 117 & 154 & -139.2 & 0.007 & 2.82 & 108.8 & 274.3 \\
\hline 14 & 124.82 & 7081308 & 0.228 & 0.576 & 0.021 & 177 & 262 & -27.6 & 0.105 & 2.23 & 104.4 & 293.8 \\
\hline 15 & 121.80 & 7012501 & 0.059 & -9 & -9 & -999 & 35 & 4.3 & 0.007 & 2.15 & 108.7 & 269.3 \\
\hline 16 & 109.02 & 8022715 & 0.105 & 0.268 & 0.006 & 197 & 82 & -29.7 & 0.007 & 1.74 & 124.9 & 277.4 \\
\hline 17 & 108.99 & 9110507 & 0.096 & -9 & -9 & -999 & 72 & 7.4 & 0.014 & 3.17 & 106.9 & 271 \\
\hline 18 & 104.48 & 10103011 & 0.237 & 0.967 & 0.017 & 312 & 276 & -11.5 & 0.105 & 2.1 & 111.2 & 276.9 \\
\hline 19 & 103.92 & 9022212 & 0.196 & 0.338 & 0.018 & 64 & 209 & -31.3 & 0.007 & 3.26 & 109.2 & 260.5 \\
\hline 20 & 102.81 & 10042210 & 0.265 & 0.829 & 0.008 & 215 & 327 & -17.5 & 0.047 & 2.99 & 107.6 & 291.3 \\
\hline 21 & 100.58 & 7081410 & 0.268 & 1.184 & 0.016 & 434 & 335 & -12.6 & 0.105 & 2.41 & 114.1 & 291.8 \\
\hline 22 & 99.85 & 9091509 & 0.216 & 0.714 & 0.023 & 205 & 241 & -14.2 & 0.105 & 1.97 & 117.8 & 295.1 \\
\hline 23 & 97.80 & 9062824 & 0.065 & -9 & -9 & -999 & 39 & 6.1 & 0.105 & 1.47 & 107.6 & 287.3 \\
\hline 24 & 97.79 & 10020315 & 0.074 & 0.345 & 0.027 & 151 & 48 & -3.7 & 0.007 & 1.03 & 127.4 & 266.2 \\
\hline 25 & 97.05 & 10110819 & 0.071 & -9 & -9 & -999 & 47 & 5.5 & 0.014 & 2.32 & 107.2 & 281.4 \\
\hline
\end{tabular}


Table 15 Meteorological Conditions for Top 25 Modeled Concentrations at North Dakota Beulah Monitor Using Beta $u^{\star}$ AERMET and Default AERMOD

\begin{tabular}{|c|c|c|c|c|c|c|c|c|c|c|c|c|}
\hline Rank & Conc. & YYMMDDHH & $\mathrm{u}^{*}$ & $\mathrm{w}^{*}$ & VPTG & $Z_{i c}$ & $z_{i m}$ & $\mathbf{L}$ & $z_{0}$ & WSPD & WDIR & temp \\
\hline 1 & 189.96 & 8020312 & 0.15 & 0.359 & 0.023 & 171 & 140 & -31.5 & 0.007 & 2.5 & 95.7 & 264.7 \\
\hline 2 & 184.16 & 7120314 & 0.19 & 0.224 & 0.032 & 41 & 199 & -63.1 & 0.007 & 3.26 & 95.1 & 272.6 \\
\hline 3 & 172.95 & 9021612 & 0.153 & 0.191 & 0.03 & 225 & 143 & -287.2 & 0.007 & 2.73 & 95.5 & 261.5 \\
\hline 4 & 158.73 & 8070609 & 0.287 & 0.889 & 0.005 & 210 & 369 & -17.7 & 0.105 & 2.68 & 332.7 & 300.4 \\
\hline 5 & 148.72 & 8021114 & 0.08 & 0.27 & 0.018 & 351 & 54 & -22.7 & 0.007 & 1.3 & 330.7 & 257.8 \\
\hline 6 & 133.47 & 8022212 & 0.204 & 0.36 & 0.018 & 76 & 221 & -34.2 & 0.007 & 3.4 & 96.7 & 272.5 \\
\hline 7 & 126.90 & 10033013 & 0.304 & 0.634 & 0.005 & 162 & 402 & -44.7 & 0.007 & 5.14 & 323.2 & 289.8 \\
\hline 8 & 121.41 & 10021516 & 0.138 & 0.32 & 0.03 & 183 & 124 & -37.3 & 0.007 & 2.32 & 324.9 & 261.4 \\
\hline 9 & 115.60 & 8031910 & 0.19 & 0.05 & 0.012 & 9 & 202 & -1291.3 & 0.007 & 3.44 & 93 & 276 \\
\hline 10 & 114.08 & 10022712 & 0.193 & 0.575 & 0.026 & 227 & 203 & -21.4 & 0.007 & 3.13 & 98.6 & 262.1 \\
\hline 11 & 113.31 & 9031216 & 0.132 & 0.493 & 0.02 & 192 & 115 & -9.2 & 0.007 & 2.01 & 209 & 264 \\
\hline 12 & 112.57 & 10110716 & 0.06 & 0.588 & 0.013 & 270 & 35 & -1 & 0.014 & 0.58 & 330 & 287.4 \\
\hline 13 & 112.43 & 10042208 & 0.184 & 0.309 & 0.012 & 46 & 190 & -24.7 & 0.047 & 2.15 & 91.1 & 286.1 \\
\hline 14 & 108.50 & 8121714 & 0.16 & 0.087 & 0.016 & 21 & 153 & -328.4 & 0.007 & 2.86 & 96.7 & 255.5 \\
\hline 15 & 108.22 & 10110616 & 0.07 & 0.608 & 0.016 & 273 & 45 & -1.1 & 0.014 & 0.72 & 318.6 & 290.4 \\
\hline 16 & 105.02 & 7031615 & 0.272 & 0.72 & 0.016 & 325 & 341 & -43.9 & 0.007 & 4.6 & 94.5 & 275.8 \\
\hline 17 & 104.78 & 10032315 & 0.146 & 0.393 & 0.012 & 311 & 134 & -40.1 & 0.007 & 2.46 & 326.3 & 274.3 \\
\hline 18 & 102.15 & 7021312 & 0.05 & 0.218 & 0.016 & 262 & 27 & -8.1 & 0.007 & 0.76 & 103 & 251.5 \\
\hline 19 & 101.73 & 8022315 & 0.174 & 0.42 & 0.013 & 112 & 174 & -20.1 & 0.007 & 2.82 & 91.8 & 278.2 \\
\hline 20 & 101.08 & 9020215 & 0.083 & 0.465 & 0.016 & 247 & 58 & -3.6 & 0.007 & 1.16 & 87.2 & 252.9 \\
\hline 21 & 99.13 & 7090309 & 0.15 & 0.786 & 0.025 & 213 & 139 & -3.7 & 0.105 & 1.12 & 333.2 & 298.3 \\
\hline 22 & 99.11 & 8081508 & 0.177 & 0.471 & 0.011 & 107 & 179 & -14.1 & 0.105 & 1.61 & 97.9 & 293.5 \\
\hline 23 & 98.10 & 8031110 & 0.234 & 0.261 & 0.005 & 46 & 273 & -84.3 & 0.007 & 4.07 & 94.8 & 281 \\
\hline 24 & 96.92 & 10051110 & 0.148 & 0.661 & 0.005 & 429 & 137 & -12.1 & 0.047 & 1.61 & 92.3 & 280.6 \\
\hline 25 & 95.51 & 8020213 & 0.131 & 0.101 & 0.026 & 145 & 114 & -798 & 0.007 & 2.37 & 92.9 & 265.1 \\
\hline
\end{tabular}

Table 16 Meteorological Conditions for Top 25 Modeled Concentrations at North Dakota DGC \#12 Monitor Using Beta $u^{*}$ AERMET and Beta AERMOD with Sigma-v $=0.5 \mathrm{~m} / \mathrm{s}$

\begin{tabular}{|c|c|c|c|c|c|c|c|c|c|c|c|c|}
\hline Rank & Conc. & YYMMDDHH & $\mathrm{u}^{*}$ & $\mathbf{w}^{*}$ & VPTG & $Z_{i c}$ & $z_{\text {im }}$ & $\mathbf{L}$ & $\mathrm{z}_{0}$ & WSPD & WDIR & temp \\
\hline 1 & 156.86 & 8070608 & 0.239 & 0.608 & 0.005 & 115 & 281 & -17.5 & 0.105 & 2.23 & 310.5 & 299.3 \\
\hline 2 & 128.44 & 10022712 & 0.193 & 0.575 & 0.026 & 227 & 203 & -21.4 & 0.007 & 3.13 & 98.6 & 262.1 \\
\hline 3 & 108.47 & 10020916 & 0.107 & 0.267 & 0.012 & 223 & 84 & -36.2 & 0.007 & 1.79 & 100.5 & 255.4 \\
\hline 4 & 108.26 & 8020314 & 0.173 & 0.494 & 0.024 & 277 & 172 & -29.6 & 0.007 & 2.86 & 97.1 & 265 \\
\hline 5 & 102.91 & 10110612 & 0.242 & 0.757 & 0.015 & 170 & 286 & -13.9 & 0.014 & 3.4 & 313.9 & 294.7 \\
\hline 6 & 100.44 & 7031617 & 0.222 & 0.38 & 0.016 & 359 & 252 & -179.2 & 0.007 & 3.93 & 100.6 & 274.6 \\
\hline 7 & 99.11 & 7081308 & 0.228 & 0.576 & 0.021 & 177 & 262 & -27.6 & 0.105 & 2.23 & 104.4 & 293.8 \\
\hline 8 & 97.74 & 8032014 & 0.25 & 0.429 & 0.013 & 345 & 300 & -171.1 & 0.007 & 4.42 & 100.3 & 273 \\
\hline 9 & 96.64 & 9022211 & 0.17 & 0.207 & 0.017 & 30 & 168 & -41.4 & 0.007 & 2.86 & 99.6 & 259.3 \\
\hline 10 & 93.42 & 8022212 & 0.204 & 0.36 & 0.018 & 76 & 221 & -34.2 & 0.007 & 3.4 & 96.7 & 272.5 \\
\hline 11 & 93.03 & 7052018 & 0.289 & 0.491 & 0.026 & 382 & 372 & -194.4 & 0.047 & 3.75 & 100.6 & 287.8 \\
\hline 12 & 88.32 & 9022414 & 0.204 & 0.588 & 0.035 & 248 & 222 & -25.8 & 0.007 & 3.35 & 200.4 & 267.2 \\
\hline 13 & 87.19 & 9021613 & 0.148 & 0.252 & 0.03 & 226 & 136 & -114.6 & 0.007 & 2.59 & 96.3 & 262.8 \\
\hline 14 & 86.37 & 8122514 & 0.21 & 0.076 & 0.013 & 17 & 232 & -874.4 & 0.007 & 3.8 & 100 & 260.1 \\
\hline 15 & 85.17 & 9090208 & 0.23 & 0.553 & 0.013 & 301 & 264 & -54.1 & 0.105 & 2.37 & 102.8 & 292.6 \\
\hline 16 & 84.84 & 8030915 & 0.128 & 0.709 & 0.005 & 387 & 111 & -5.8 & 0.007 & 1.88 & 317.3 & 276.8 \\
\hline 17 & 84.63 & 7030916 & 0.226 & 0.591 & 0.008 & 274 & 259 & -38.5 & 0.007 & 3.8 & 315.3 & 280 \\
\hline 18 & 80.24 & 10042110 & 0.276 & 0.822 & 0.008 & 216 & 349 & -20.6 & 0.047 & 3.17 & 102.5 & 287.5 \\
\hline 19 & 79.36 & 8051809 & 0.262 & 0.841 & 0.012 & 257 & 322 & -19.5 & 0.047 & 2.99 & 97 & 292.2 \\
\hline 20 & 79.30 & 10072010 & 0.407 & 1.426 & 0.005 & 684 & 625 & -39.8 & 0.105 & 4.11 & 317.2 & 295.5 \\
\hline 21 & 77.99 & 9020413 & 0.143 & 0.426 & 0.031 & 173 & 131 & -16.3 & 0.007 & 2.28 & 195.8 & 271.7 \\
\hline 22 & 76.32 & 7120314 & 0.19 & 0.224 & 0.032 & 41 & 199 & -63.1 & 0.007 & 3.26 & 95.1 & 272.6 \\
\hline 23 & 76.28 & 8081508 & 0.177 & 0.471 & 0.011 & 107 & 179 & -14.1 & 0.105 & 1.61 & 97.9 & 293.5 \\
\hline 24 & 75.29 & 10052709 & 0.246 & 0.474 & 0.008 & 181 & 316 & -63.4 & 0.029 & 3.36 & 102 & 293.1 \\
\hline 25 & 75.01 & 10051210 & 0.306 & 0.564 & 0.005 & 268 & 415 & -106.7 & 0.047 & 3.89 & 99.8 & 279.3 \\
\hline
\end{tabular}


Table 17 Meteorological Conditions for Top 25 Modeled Concentrations at North Dakota DGC \#14 Monitor Using Beta $u^{*}$ AERMET and Beta AERMOD with Sigma-v $=0.5 \mathrm{~m} / \mathrm{s}$

\begin{tabular}{|c|c|c|c|c|c|c|c|c|c|c|c|c|}
\hline Rank & Conc. & YYMMDDHH & $\mathrm{u}^{*}$ & $\mathrm{w}^{*}$ & VPTG & $z_{i c}$ & $z_{\text {im }}$ & $\mathbf{L}$ & $\mathrm{z}_{0}$ & WSPD & WDIR & temp \\
\hline 1 & 191.04 & 10032717 & 0.188 & 0.527 & 0.009 & 348 & 199 & -39.8 & 0.007 & 3.17 & 262.9 & 274.1 \\
\hline 2 & 188.77 & 8071609 & 0.246 & 0.894 & 0.012 & 226 & 293 & -11.8 & 0.105 & 2.19 & 257.5 & 298.6 \\
\hline 3 & 155.86 & 7030611 & 0.075 & 0.525 & 0.047 & 267 & 50 & -2 & 0.007 & 0.98 & 262.2 & 272.1 \\
\hline 4 & 131.09 & 10040317 & 0.434 & 1.382 & 0.005 & 1486 & 686 & -115 & 0.047 & 5.54 & 258 & 282.1 \\
\hline 5 & 130.90 & 10030515 & 0.104 & 0.33 & 0.038 & 322 & 80 & -25 & 0.007 & 1.7 & 109.5 & 270.6 \\
\hline 6 & 118.51 & 9020812 & 0.19 & 0.234 & 0.027 & 36 & 198 & -48.3 & 0.007 & 3.22 & 107.9 & 271.6 \\
\hline 7 & 114.23 & 8112514 & 0.197 & 0.634 & 0.025 & 226 & 215 & -17 & 0.014 & 2.82 & 263.2 & 279.3 \\
\hline 8 & 105.34 & 10031316 & 0.16 & 0.206 & 0.018 & 117 & 154 & -139.2 & 0.007 & 2.82 & 108.8 & 274.3 \\
\hline 9 & 103.18 & 7081308 & 0.228 & 0.576 & 0.021 & 177 & 262 & -27.6 & 0.105 & 2.23 & 104.4 & 293.8 \\
\hline 10 & 102.08 & 8071408 & 0.249 & 0.734 & 0.006 & 216 & 299 & -21.2 & 0.105 & 2.37 & 267.5 & 298.9 \\
\hline 11 & 99.90 & 7052811 & 0.202 & 0.767 & 0.013 & 301 & 218 & -13.8 & 0.047 & 2.23 & 265.1 & 288.7 \\
\hline 12 & 93.92 & 7060909 & 0.288 & 1.235 & 0.006 & 356 & 374 & -11.3 & 0.105 & 2.55 & 267.6 & 294.9 \\
\hline 13 & 93.43 & 10042209 & 0.229 & 0.561 & 0.011 & 103 & 263 & -17.6 & 0.047 & 2.59 & 105.8 & 288.8 \\
\hline 14 & 93.26 & 9022212 & 0.196 & 0.338 & 0.018 & 64 & 209 & -31.3 & 0.007 & 3.26 & 109.2 & 260.5 \\
\hline 15 & 92.64 & 8020313 & 0.171 & 0.467 & 0.024 & 235 & 169 & -28.7 & 0.007 & 2.82 & 104.2 & 264.9 \\
\hline 16 & 90.38 & 7091510 & 0.236 & 0.934 & 0.015 & 259 & 275 & -10.4 & 0.105 & 2.06 & 261.8 & 288.9 \\
\hline 17 & 86.40 & 10033115 & 0.302 & 0.614 & 0.005 & 331 & 399 & -98.5 & 0.007 & 5.27 & 260.4 & 286.6 \\
\hline 18 & 84.73 & 10111214 & 0.213 & 0.899 & 0.006 & 343 & 237 & -11.5 & 0.014 & 2.95 & 272.2 & 281.3 \\
\hline 19 & 84.37 & 8022316 & 0.22 & 0.342 & 0.009 & 118 & 247 & -78 & 0.007 & 3.8 & 106.8 & 276.9 \\
\hline 20 & 84.35 & 8032015 & 0.249 & 0.402 & 0.013 & 349 & 298 & -207.2 & 0.007 & 4.42 & 109.4 & 273.4 \\
\hline 21 & 84.01 & 8031111 & 0.174 & 0.411 & 0.009 & 83 & 175 & -15.7 & 0.007 & 2.77 & 110.7 & 283.2 \\
\hline 22 & 81.84 & 10110713 & 0.098 & 0.818 & 0.019 & 205 & 74 & -1 & 0.014 & 0.98 & 240.8 & 289.6 \\
\hline 23 & 81.58 & 10080210 & 0.324 & 1.154 & 0.006 & 374 & 444 & -20.8 & 0.105 & 3.08 & 264.9 & 300.1 \\
\hline 24 & 77.19 & 7121713 & 0.162 & 0.109 & 0.012 & 19 & 156 & -156.4 & 0.007 & 2.86 & 104.4 & 274.2 \\
\hline 25 & 74.92 & 7031612 & 0.195 & 0.611 & 0.019 & 217 & 207 & -17.7 & 0.007 & 3.13 & 103.5 & 274.3 \\
\hline
\end{tabular}

Table 18 Meteorological Conditions for Top 25 Modeled Concentrations at North Dakota DGC \#16 Monitor Using Beta $u^{*}$ AERMET and Beta AERMOD with Sigma-v $=0.5 \mathrm{~m} / \mathrm{s}$

\begin{tabular}{|c|c|c|c|c|c|c|c|c|c|c|c|c|}
\hline Rank & Conc. & YYMMDDHH & $u^{*}$ & $\mathrm{w}^{*}$ & VPTG & $Z_{i c}$ & $z_{\text {im }}$ & $\mathbf{L}$ & $z_{0}$ & WSPD & WDIR & temp \\
\hline 1 & 190.51 & 10040315 & 0.46 & 1.264 & 0.005 & 1312 & 750 & -158.2 & 0.047 & 5.94 & 150.5 & 282.5 \\
\hline 2 & 140.09 & 7081609 & 0.154 & 0.831 & 0.009 & 239 & 146 & -3.8 & 0.105 & 1.16 & 139.9 & 292.8 \\
\hline 3 & 138.37 & 10030511 & 0.095 & 0.535 & 0.038 & 296 & 70 & -4.1 & 0.007 & 1.34 & 114.7 & 268.7 \\
\hline 4 & 128.98 & 7061608 & 0.144 & 0.964 & 0.009 & 269 & 131 & -2.2 & 0.105 & 0.98 & 133 & 290.4 \\
\hline 5 & 127.88 & 10072516 & 0.509 & 2.095 & 0.01 & 1813 & 877 & -65 & 0.105 & 5.32 & 150.4 & 303.2 \\
\hline 6 & 125.88 & 9061708 & 0.272 & 0.821 & 0.01 & 403 & 341 & -36.8 & 0.105 & 2.73 & 141.8 & 289.3 \\
\hline 7 & 121.08 & 8100212 & 0.239 & 1.117 & 0.007 & 327 & 280 & -8 & 0.105 & 2.01 & 127.7 & 295.1 \\
\hline 8 & 114.31 & 9062018 & 0.482 & 1.666 & 0.005 & 2273 & 833 & -137.6 & 0.105 & 5.23 & 132.9 & 298.5 \\
\hline 9 & 112.02 & 9080710 & 0.139 & 0.827 & 0.009 & 380 & 125 & -4.5 & 0.105 & 1.07 & 152.1 & 292.1 \\
\hline 10 & 111.36 & 10072119 & 0.383 & 1.143 & 0.005 & 2003 & 569 & -188.5 & 0.105 & 4.2 & 148.4 & 294 \\
\hline 11 & 108.99 & 10021213 & 0.152 & 0.623 & 0.019 & 329 & 142 & -11.9 & 0.007 & 2.37 & 125 & 264.6 \\
\hline 12 & 101.31 & 9020812 & 0.19 & 0.234 & 0.027 & 36 & 198 & -48.3 & 0.007 & 3.22 & 107.9 & 271.6 \\
\hline 13 & 98.16 & 10062808 & 0.118 & 0.928 & 0.008 & 417 & 98 & -2.2 & 0.105 & 0.8 & 147.5 & 289 \\
\hline 14 & 95.63 & 10080911 & 0.326 & 1.275 & 0.006 & 415 & 447 & -17.4 & 0.105 & 3.04 & 130.8 & 303.5 \\
\hline 15 & 95.16 & 8082602 & 0.811 & -9 & -9 & -999 & 1757 & 678.4 & 0.105 & 9.39 & 150.1 & 292 \\
\hline 16 & 93.02 & 8013115 & 0.36 & 0.364 & 0.02 & 211 & 519 & -513.7 & 0.007 & 6.48 & 153.7 & 272 \\
\hline 17 & 92.38 & 10103013 & 0.238 & 1.182 & 0.011 & 397 & 279 & -8.1 & 0.105 & 2.01 & 120.4 & 278.4 \\
\hline 18 & 90.42 & 7100411 & 0.157 & 0.979 & 0.005 & 276 & 150 & -2.9 & 0.105 & 1.12 & 138.7 & 286 \\
\hline 19 & 89.51 & 9090618 & 0.521 & 1.146 & 0.008 & 1098 & 926 & -258 & 0.105 & 5.77 & 131.8 & 298.5 \\
\hline 20 & 89.32 & 7081308 & 0.228 & 0.576 & 0.021 & 177 & 262 & -27.6 & 0.105 & 2.23 & 104.4 & 293.8 \\
\hline 21 & 87.82 & 10031316 & 0.16 & 0.206 & 0.018 & 117 & 154 & -139.2 & 0.007 & 2.82 & 108.8 & 274.3 \\
\hline 22 & 86.10 & 10110716 & 0.06 & 0.588 & 0.013 & 270 & 35 & -1 & 0.014 & 0.58 & 330 & 287.4 \\
\hline 23 & 84.59 & 8092212 & 0.284 & 1.713 & 0.011 & 749 & 363 & -8.5 & 0.105 & 2.41 & 125.3 & 295.1 \\
\hline 24 & 80.82 & 10081211 & 0.279 & 1.366 & 0.006 & 519 & 370 & -11.1 & 0.105 & 2.46 & 140.8 & 300.9 \\
\hline 25 & 80.15 & 8020313 & 0.171 & 0.467 & 0.024 & 235 & 169 & -28.7 & 0.007 & 2.82 & 104.2 & 264.9 \\
\hline
\end{tabular}


Table 19 Meteorological Conditions for Top 25 Modeled Concentrations at North Dakota DGC \#17 Monitor Using Beta $u^{*}$ AERMET and Beta AERMOD with Sigma-v $=0.5 \mathrm{~m} / \mathrm{s}$

\begin{tabular}{|c|c|c|c|c|c|c|c|c|c|c|c|c|}
\hline Rank & Conc. & YYMMDDHH & $u^{*}$ & $\mathrm{w}^{*}$ & VPTG & $z_{i c}$ & $z_{\text {im }}$ & $\mathbf{L}$ & $z_{0}$ & WSPD & WDIR & temp \\
\hline 1 & 214.16 & 8013107 & 0.05 & -9 & -9 & -999 & 27 & 3.5 & 0.007 & 1.83 & 131.4 & 252.7 \\
\hline 2 & 154.09 & 8020215 & 0.156 & 0.427 & 0.029 & 200 & 147 & -24.2 & 0.007 & 2.55 & 124.9 & 266.9 \\
\hline 3 & 152.04 & 10030515 & 0.104 & 0.33 & 0.038 & 322 & 80 & -25 & 0.007 & 1.7 & 109.5 & 270.6 \\
\hline 4 & 151.97 & 8013022 & 0.124 & -9 & -9 & -999 & 106 & 11 & 0.007 & 3.66 & 127.6 & 252.7 \\
\hline 5 & 132.94 & 10021212 & 0.107 & 0.513 & 0.019 & 232 & 85 & -5.3 & 0.007 & 1.56 & 121.1 & 263.8 \\
\hline 6 & 131.54 & 9032017 & 0.136 & 0.466 & 0.023 & 307 & 120 & -19 & 0.007 & 2.19 & 113.8 & 279.8 \\
\hline 7 & 129.06 & 9031615 & 0.138 & 0.6 & 0.019 & 184 & 123 & -5.6 & 0.007 & 2.01 & 111.3 & 279.6 \\
\hline 8 & 125.96 & 10080810 & 0.292 & 1.03 & 0.005 & 269 & 378 & -15.3 & 0.105 & 2.68 & 125.5 & 304 \\
\hline 9 & 125.58 & 7031613 & 0.192 & 0.533 & 0.018 & 250 & 201 & -29.1 & 0.007 & 3.17 & 115.7 & 275.1 \\
\hline 10 & 109.08 & 7081308 & 0.228 & 0.576 & 0.021 & 177 & 262 & -27.6 & 0.105 & 2.23 & 104.4 & 293.8 \\
\hline 11 & 102.07 & 9020812 & 0.19 & 0.234 & 0.027 & 36 & 198 & -48.3 & 0.007 & 3.22 & 107.9 & 271.6 \\
\hline 12 & 97.80 & 9110507 & 0.096 & -9 & -9 & -999 & 72 & 7.4 & 0.014 & 3.17 & 106.9 & 271 \\
\hline 13 & 95.32 & 9091509 & 0.216 & 0.714 & 0.023 & 205 & 241 & -14.2 & 0.105 & 1.97 & 117.8 & 295.1 \\
\hline 14 & 93.87 & 10103011 & 0.237 & 0.967 & 0.017 & 312 & 276 & -11.5 & 0.105 & 2.1 & 111.2 & 276.9 \\
\hline 15 & 92.42 & 10042210 & 0.265 & 0.829 & 0.008 & 215 & 327 & -17.5 & 0.047 & 2.99 & 107.6 & 291.3 \\
\hline 16 & 92.13 & 7081410 & 0.268 & 1.184 & 0.016 & 434 & 335 & -12.6 & 0.105 & 2.41 & 114.1 & 291.8 \\
\hline 17 & 91.30 & 7110811 & 0.204 & 0.947 & 0.011 & 334 & 221 & -8.3 & 0.014 & 2.73 & 117.2 & 275.4 \\
\hline 18 & 88.01 & 10080109 & 0.262 & 0.997 & 0.008 & 345 & 323 & -15.6 & 0.105 & 2.41 & 106.1 & 294.9 \\
\hline 19 & 85.02 & 9022212 & 0.196 & 0.338 & 0.018 & 64 & 209 & -31.3 & 0.007 & 3.26 & 109.2 & 260.5 \\
\hline 20 & 84.65 & 7042310 & 0.253 & 0.854 & 0.005 & 239 & 305 & -15.5 & 0.047 & 2.82 & 116.4 & 287.1 \\
\hline 21 & 84.37 & 8110913 & 0.263 & 0.846 & 0.027 & 362 & 324 & -27.3 & 0.014 & 3.89 & 110.7 & 261.7 \\
\hline 22 & 84.31 & 8022914 & 0.124 & 0.622 & 0.007 & 261 & 104 & -5.1 & 0.007 & 1.79 & 126.5 & 276.9 \\
\hline 23 & 83.81 & 7070111 & 0.24 & 1.206 & 0.016 & 468 & 285 & -9.2 & 0.105 & 2.06 & 114.1 & 298.6 \\
\hline 24 & 83.80 & 9061609 & 0.327 & 0.738 & 0.011 & 341 & 449 & -74.2 & 0.105 & 3.44 & 116.4 & 291.8 \\
\hline 25 & 83.09 & 8020313 & 0.171 & 0.467 & 0.024 & 235 & 169 & -28.7 & 0.007 & 2.82 & 104.2 & 264.9 \\
\hline
\end{tabular}

Table 20 Meteorological Conditions for Top 25 Modeled Concentrations at North Dakota Beulah Monitor Using Beta $u^{\star}$ AERMET and Beta AERMOD with Sigma-v $=0.5 \mathrm{~m} / \mathrm{s}$

\begin{tabular}{|c|c|c|c|c|c|c|c|c|c|c|c|c|}
\hline Rank & Conc. & YYMMDDHH & $\mathrm{u}^{*}$ & $\mathrm{w}^{*}$ & VPTG & $Z_{i c}$ & $z_{i m}$ & $\mathbf{L}$ & $z_{0}$ & WSPD & WDIR & temp \\
\hline 1 & 155.03 & 8070609 & 0.287 & 0.889 & 0.005 & 210 & 369 & -17.7 & 0.105 & 2.68 & 332.7 & 300.4 \\
\hline 2 & 135.93 & 7120314 & 0.19 & 0.224 & 0.032 & 41 & 199 & -63.1 & 0.007 & 3.26 & 95.1 & 272.6 \\
\hline 3 & 126.58 & 8020312 & 0.15 & 0.359 & 0.023 & 171 & 140 & -31.5 & 0.007 & 2.5 & 95.7 & 264.7 \\
\hline 4 & 125.08 & 10033013 & 0.304 & 0.634 & 0.005 & 162 & 402 & -44.7 & 0.007 & 5.14 & 323.2 & 289.8 \\
\hline 5 & 112.07 & 8022212 & 0.204 & 0.36 & 0.018 & 76 & 221 & -34.2 & 0.007 & 3.4 & 96.7 & 272.5 \\
\hline 6 & 108.81 & 9021612 & 0.153 & 0.191 & 0.03 & 225 & 143 & -287.2 & 0.007 & 2.73 & 95.5 & 261.5 \\
\hline 7 & 103.39 & 10022712 & 0.193 & 0.575 & 0.026 & 227 & 203 & -21.4 & 0.007 & 3.13 & 98.6 & 262.1 \\
\hline 8 & 98.37 & 7031615 & 0.272 & 0.72 & 0.016 & 325 & 341 & -43.9 & 0.007 & 4.6 & 94.5 & 275.8 \\
\hline 9 & 95.21 & 7090309 & 0.15 & 0.786 & 0.025 & 213 & 139 & -3.7 & 0.105 & 1.12 & 333.2 & 298.3 \\
\hline 10 & 91.31 & 9031216 & 0.132 & 0.493 & 0.02 & 192 & 115 & -9.2 & 0.007 & 2.01 & 209 & 264 \\
\hline 11 & 90.38 & 10021516 & 0.138 & 0.32 & 0.03 & 183 & 124 & -37.3 & 0.007 & 2.32 & 324.9 & 261.4 \\
\hline 12 & 89.77 & 10110613 & 0.223 & 0.844 & 0.019 & 217 & 253 & -10 & 0.014 & 3.04 & 327.9 & 295.1 \\
\hline 13 & 88.74 & 8031910 & 0.19 & 0.05 & 0.012 & 9 & 202 & -1291.3 & 0.007 & 3.44 & 93 & 276 \\
\hline 14 & 87.29 & 10042208 & 0.184 & 0.309 & 0.012 & 46 & 190 & -24.7 & 0.047 & 2.15 & 91.1 & 286.1 \\
\hline 15 & 86.72 & 8031110 & 0.234 & 0.261 & 0.005 & 46 & 273 & -84.3 & 0.007 & 4.07 & 94.8 & 281 \\
\hline 16 & 86.25 & 8032013 & 0.255 & 0.427 & 0.014 & 337 & 308 & -179.1 & 0.007 & 4.51 & 96.9 & 272.5 \\
\hline 17 & 84.07 & 8110112 & 0.252 & 0.721 & 0.019 & 206 & 303 & -21.8 & 0.014 & 3.66 & 205.4 & 284.5 \\
\hline 18 & 83.95 & 8051809 & 0.262 & 0.841 & 0.012 & 257 & 322 & -19.5 & 0.047 & 2.99 & 97 & 292.2 \\
\hline 19 & 83.91 & 9022414 & 0.204 & 0.588 & 0.035 & 248 & 222 & -25.8 & 0.007 & 3.35 & 200.4 & 267.2 \\
\hline 20 & 82.52 & 8022315 & 0.174 & 0.42 & 0.013 & 112 & 174 & -20.1 & 0.007 & 2.82 & 91.8 & 278.2 \\
\hline 21 & 81.73 & 9031415 & 0.187 & 0.606 & 0.021 & 219 & 195 & -16.2 & 0.007 & 2.99 & 209.2 & 277.9 \\
\hline 22 & 80.83 & 10032315 & 0.146 & 0.393 & 0.012 & 311 & 134 & -40.1 & 0.007 & 2.46 & 326.3 & 274.3 \\
\hline 23 & 79.48 & 10020916 & 0.107 & 0.267 & 0.012 & 223 & 84 & -36.2 & 0.007 & 1.79 & 100.5 & 255.4 \\
\hline 24 & 78.70 & 10051110 & 0.148 & 0.661 & 0.005 & 429 & 137 & -12.1 & 0.047 & 1.61 & 92.3 & 280.6 \\
\hline 25 & 78.35 & 10030411 & 0.132 & 0.481 & 0.023 & 222 & 116 & -11.6 & 0.007 & 2.06 & 211.2 & 271.4 \\
\hline
\end{tabular}


Table 21 Meteorological Conditions for Top 25 Modeled Concentrations at North Dakota DGC \#12 Monitor Using Beta $u^{\star}$ AERMET and SHARP

\begin{tabular}{|c|c|c|c|c|c|c|c|c|c|c|c|c|}
\hline 1 & 120.97 & 8020214 & 0.158 & 0.102 & 0.026 & 146 & 151 & -1372 & 0.007 & 2.86 & 99.5 & 266 \\
\hline 2 & 116.42 & 10022713 & 0.208 & 0.672 & 0.028 & 306 & 228 & -22.8 & 0.007 & 3.4 & 101.3 & 262.9 \\
\hline 3 & 112.22 & 8070608 & 0.239 & 0.608 & 0.005 & 115 & 281 & -17.5 & 0.105 & 2.23 & 310.5 & 299.3 \\
\hline 4 & 104.46 & 8022212 & 0.204 & 0.36 & 0.018 & 76 & 221 & -34.2 & 0.007 & 3.4 & 96.7 & 272.5 \\
\hline 5 & 97.85 & 7031612 & 0.195 & 0.611 & 0.019 & 217 & 207 & -17.7 & 0.007 & 3.13 & 103.5 & 274.3 \\
\hline 6 & 86.60 & 9020215 & 0.083 & 0.465 & 0.016 & 247 & 58 & -3.6 & 0.007 & 1.16 & 87.2 & 252.9 \\
\hline 7 & 84.36 & 8020314 & 0.173 & 0.494 & 0.024 & 277 & 172 & -29.6 & 0.007 & 2.86 & 97.1 & 265 \\
\hline 8 & 84.28 & 7081308 & 0.228 & 0.576 & 0.021 & 177 & 262 & -27.6 & 0.105 & 2.23 & 104.4 & 293.8 \\
\hline 9 & 78.83 & 10020916 & 0.107 & 0.267 & 0.012 & 223 & 84 & -36.2 & 0.007 & 1.79 & 100.5 & 255.4 \\
\hline 10 & 78.50 & 10110612 & 0.242 & 0.757 & 0.015 & 170 & 286 & -13.9 & 0.014 & 3.4 & 313.9 & 294.7 \\
\hline 11 & 77.32 & 10021212 & 0.107 & 0.513 & 0.019 & 232 & 85 & -5.3 & 0.007 & 1.56 & 121.1 & 263.8 \\
\hline 12 & 77.20 & 10042209 & 0.229 & 0.561 & 0.011 & 103 & 263 & -17.6 & 0.047 & 2.59 & 105.8 & 288.8 \\
\hline 13 & 74.03 & 9090208 & 0.23 & 0.553 & 0.013 & 301 & 264 & -54.1 & 0.105 & 2.37 & 102.8 & 292.6 \\
\hline 14 & 69.29 & 9021612 & 0.153 & 0.191 & 0.03 & 225 & 143 & -287.2 & 0.007 & 2.73 & 95.5 & 261.5 \\
\hline 15 & 68.91 & 8121714 & 0.16 & 0.087 & 0.016 & 21 & 153 & -328.4 & 0.007 & 2.86 & 96.7 & 255.5 \\
\hline 16 & 67.60 & 8032014 & 0.25 & 0.429 & 0.013 & 345 & 300 & -171.1 & 0.007 & 4.42 & 100.3 & 273 \\
\hline 17 & 67.51 & 9112915 & 0.095 & 0.334 & 0.007 & 252 & 91 & -14.5 & 0.014 & 1.34 & 320 & 275.1 \\
\hline 18 & 66.82 & 8032616 & 0.189 & 0.345 & 0.005 & 281 & 197 & -115.4 & 0.007 & 3.31 & 113 & 279.3 \\
\hline 19 & 65.08 & 10030515 & 0.104 & 0.33 & 0.038 & 322 & 80 & -25 & 0.007 & 1.7 & 109.5 & 270.6 \\
\hline 20 & 64.80 & 10051110 & 0.148 & 0.661 & 0.005 & 429 & 137 & -12.1 & 0.047 & 1.61 & 92.3 & 280.6 \\
\hline 21 & 64.55 & 9111310 & 0.163 & 0.594 & 0.011 & 263 & 157 & -13.5 & 0.014 & 2.28 & 305.6 & 275.8 \\
\hline 22 & 63.48 & 7021314 & 0.067 & 0.275 & 0.016 & 266 & 42 & -9.7 & 0.007 & 1.03 & 70.2 & 252.3 \\
\hline 23 & 63.19 & 8031117 & 0.222 & 0.352 & 0.005 & 194 & 250 & -122 & 0.007 & 3.89 & 104.6 & 284.6 \\
\hline 24 & 62.78 & 10072410 & 0.212 & 0.784 & 0.006 & 231 & 235 & -11.4 & 0.105 & 1.88 & 359 & 293.6 \\
\hline 25 & 62.41 & 8051809 & 0.262 & 0.841 & 0.012 & 257 & 322 & -19.5 & 0.047 & 2.99 & 97 & 292.2 \\
\hline
\end{tabular}

Table 22 Meteorological Conditions for Top 25 Modeled Concentrations at North Dakota DGC \#14 Monitor Using Beta $u^{\star}$ AERMET and SHARP

\begin{tabular}{|c|c|c|c|c|c|c|c|c|c|c|c|c|}
\hline Rank & Conc. & YYMMDDHH & $\mathrm{u}^{*}$ & $\mathrm{w}^{*}$ & VPTG & $Z_{i c}$ & $z_{i m}$ & $\mathbf{L}$ & $\mathrm{z}_{0}$ & WSPD & WDIR & temp \\
\hline 1 & 103.97 & 10033116 & 0.259 & 0.746 & 0.005 & 366 & 316 & -38.2 & 0.007 & 4.34 & 249.1 & 286.1 \\
\hline 2 & 99.99 & 10032717 & 0.188 & 0.527 & 0.009 & 348 & 199 & -39.8 & 0.007 & 3.17 & 262.9 & 274.1 \\
\hline 3 & 92.51 & 7081308 & 0.228 & 0.576 & 0.021 & 177 & 262 & -27.6 & 0.105 & 2.23 & 104.4 & 293.8 \\
\hline 4 & 91.91 & 9031416 & 0.17 & 0.541 & 0.02 & 230 & 168 & -17.9 & 0.007 & 2.73 & 224 & 277.3 \\
\hline 5 & 90.30 & 10110713 & 0.098 & 0.818 & 0.019 & 205 & 74 & -1 & 0.014 & 0.98 & 240.8 & 289.6 \\
\hline 6 & 88.22 & 7030611 & 0.075 & 0.525 & 0.047 & 267 & 50 & -2 & 0.007 & 0.98 & 262.2 & 272.1 \\
\hline 7 & 86.60 & 10031316 & 0.16 & 0.206 & 0.018 & 117 & 154 & -139.2 & 0.007 & 2.82 & 108.8 & 274.3 \\
\hline 8 & 82.57 & 8071609 & 0.246 & 0.894 & 0.012 & 226 & 293 & -11.8 & 0.105 & 2.19 & 257.5 & 298.6 \\
\hline 9 & 82.00 & 8020215 & 0.156 & 0.427 & 0.029 & 200 & 147 & -24.2 & 0.007 & 2.55 & 124.9 & 266.9 \\
\hline 10 & 78.65 & 8032616 & 0.189 & 0.345 & 0.005 & 281 & 197 & -115.4 & 0.007 & 3.31 & 113 & 279.3 \\
\hline 11 & 76.98 & 7090309 & 0.15 & 0.786 & 0.025 & 213 & 139 & -3.7 & 0.105 & 1.12 & 333.2 & 298.3 \\
\hline 12 & 76.66 & 10040317 & 0.434 & 1.382 & 0.005 & 1486 & 686 & -115 & 0.047 & 5.54 & 258 & 282.1 \\
\hline 13 & 75.55 & 9020812 & 0.19 & 0.234 & 0.027 & 36 & 198 & -48.3 & 0.007 & 3.22 & 107.9 & 271.6 \\
\hline 14 & 74.94 & 10022714 & 0.174 & 0.335 & 0.027 & 307 & 174 & -107.1 & 0.007 & 3.04 & 100.9 & 263.1 \\
\hline 15 & 74.33 & 7030812 & 0.119 & 0.638 & 0.043 & 281 & 99 & -4.6 & 0.007 & 1.7 & 245.8 & 279.4 \\
\hline 16 & 73.79 & 8022316 & 0.22 & 0.342 & 0.009 & 118 & 247 & -78 & 0.007 & 3.8 & 106.8 & 276.9 \\
\hline 17 & 73.65 & 10121414 & 0.243 & 0.307 & 0.039 & 134 & 287 & -166 & 0.007 & 4.29 & 115.3 & 262.9 \\
\hline 18 & 72.18 & 10111214 & 0.213 & 0.899 & 0.006 & 343 & 237 & -11.5 & 0.014 & 2.95 & 272.2 & 281.3 \\
\hline 19 & 71.22 & 9022416 & 0.157 & 0.448 & 0.034 & 260 & 149 & -28 & 0.007 & 2.59 & 279.8 & 267.3 \\
\hline 20 & 70.77 & 10030515 & 0.104 & 0.33 & 0.038 & 322 & 80 & -25 & 0.007 & 1.7 & 109.5 & 270.6 \\
\hline 21 & 70.23 & 8112514 & 0.197 & 0.634 & 0.025 & 226 & 215 & -17 & 0.014 & 2.82 & 263.2 & 279.3 \\
\hline 22 & 69.33 & 7052811 & 0.202 & 0.767 & 0.013 & 301 & 218 & -13.8 & 0.047 & 2.23 & 265.1 & 288.7 \\
\hline 23 & 68.39 & 10112415 & 0.523 & 0.845 & 0.01 & 564 & 906 & -333.9 & 0.014 & 8.45 & 268.4 & 254.8 \\
\hline 24 & 68.18 & 10021216 & 0.151 & 0.319 & 0.019 & 359 & 141 & -96.4 & 0.007 & 2.64 & 118.4 & 265.5 \\
\hline 25 & 66.31 & 8032016 & 0.23 & 0.527 & 0.013 & 358 & 265 & -74.9 & 0.007 & 3.98 & 112.5 & 273.3 \\
\hline
\end{tabular}


Table 23 Meteorological Conditions for Top 25 Modeled Concentrations at North Dakota DGC \#16 Monitor Using Beta $u^{*}$ AERMET and SHARP

\begin{tabular}{|c|c|c|c|c|c|c|c|c|c|c|c|c|}
\hline Rank & Conc. & YYMMDDHH & $\mathrm{u}^{*}$ & $\mathrm{w}^{*}$ & VPTG & $Z_{i c}$ & $z_{i m}$ & $\mathbf{L}$ & $\mathrm{z}_{0}$ & WSPD & WDIR & temp \\
\hline 1 & 167.25 & 10030511 & 0.095 & 0.535 & 0.038 & 296 & 70 & -4.1 & 0.007 & 1.34 & 114.7 & 268.7 \\
\hline 2 & 165.17 & 10040315 & 0.46 & 1.264 & 0.005 & 1312 & 750 & -158.2 & 0.047 & 5.94 & 150.5 & 282.5 \\
\hline 3 & 122.75 & 10021213 & 0.152 & 0.623 & 0.019 & 329 & 142 & -11.9 & 0.007 & 2.37 & 125 & 264.6 \\
\hline 4 & 119.66 & 10072516 & 0.509 & 2.095 & 0.01 & 1813 & 877 & -65 & 0.105 & 5.32 & 150.4 & 303.2 \\
\hline 5 & 111.86 & 9061708 & 0.272 & 0.821 & 0.01 & 403 & 341 & -36.8 & 0.105 & 2.73 & 141.8 & 289.3 \\
\hline 6 & 106.40 & 9062018 & 0.482 & 1.666 & 0.005 & 2273 & 833 & -137.6 & 0.105 & 5.23 & 132.9 & 298.5 \\
\hline 7 & 105.07 & 10072113 & 0.493 & 2.004 & 0.005 & 1314 & 852 & -48.8 & 0.105 & 5.05 & 135.7 & 296.4 \\
\hline 8 & 92.99 & 10103010 & 0.138 & 0.72 & 0.019 & 266 & 123 & -4.7 & 0.105 & 1.07 & 87.2 & 275.4 \\
\hline 9 & 87.87 & 8082601 & 0.839 & -9 & -9 & -999 & 1840 & 751.8 & 0.105 & 9.7 & 148.3 & 292.3 \\
\hline 10 & 86.56 & 8013115 & 0.36 & 0.364 & 0.02 & 211 & 519 & -513.7 & 0.007 & 6.48 & 153.7 & 272 \\
\hline 11 & 84.81 & 7081410 & 0.268 & 1.184 & 0.016 & 434 & 335 & -12.6 & 0.105 & 2.41 & 114.1 & 291.8 \\
\hline 12 & 83.76 & 7061608 & 0.144 & 0.964 & 0.009 & 269 & 131 & -2.2 & 0.105 & 0.98 & 133 & 290.4 \\
\hline 13 & 82.45 & 9080710 & 0.139 & 0.827 & 0.009 & 380 & 125 & -4.5 & 0.105 & 1.07 & 152.1 & 292.1 \\
\hline 14 & 81.21 & 7081308 & 0.228 & 0.576 & 0.021 & 177 & 262 & -27.6 & 0.105 & 2.23 & 104.4 & 293.8 \\
\hline 15 & 77.76 & 10080911 & 0.326 & 1.275 & 0.006 & 415 & 447 & -17.4 & 0.105 & 3.04 & 130.8 & 303.5 \\
\hline 16 & 75.97 & 9090618 & 0.521 & 1.146 & 0.008 & 1098 & 926 & -258 & 0.105 & 5.77 & 131.8 & 298.5 \\
\hline 17 & 75.87 & 10080810 & 0.292 & 1.03 & 0.005 & 269 & 378 & -15.3 & 0.105 & 2.68 & 125.5 & 304 \\
\hline 18 & 75.20 & 8020215 & 0.156 & 0.427 & 0.029 & 200 & 147 & -24.2 & 0.007 & 2.55 & 124.9 & 266.9 \\
\hline 19 & 73.81 & 8082709 & 0.283 & 0.992 & 0.007 & 479 & 366 & -27.9 & 0.105 & 2.77 & 152.9 & 287.8 \\
\hline 20 & 73.80 & 8100212 & 0.239 & 1.117 & 0.007 & 327 & 280 & -8 & 0.105 & 2.01 & 127.7 & 295.1 \\
\hline 21 & 72.42 & 7061113 & 0.566 & 2.403 & 0.005 & 1382 & 1022 & -45.2 & 0.105 & 5.77 & 130.6 & 302.1 \\
\hline 22 & 72.01 & 10031316 & 0.16 & 0.206 & 0.018 & 117 & 154 & -139.2 & 0.007 & 2.82 & 108.8 & 274.3 \\
\hline 23 & 71.70 & 10110714 & 0.08 & 0.841 & 0.016 & 241 & 54 & -1 & 0.014 & 0.72 & 83 & 289.9 \\
\hline 24 & 70.99 & 7030612 & 0.099 & 0.63 & 0.047 & 294 & 74 & -2.8 & 0.007 & 1.34 & 188.9 & 273.3 \\
\hline 25 & 70.66 & 7110811 & 0.204 & 0.947 & 0.011 & 334 & 221 & -8.3 & 0.014 & 2.73 & 117.2 & 275.4 \\
\hline
\end{tabular}

Table 24 Meteorological Conditions for Top 25 Modeled Concentrations at North Dakota DGC \#17 Monitor Using Beta $u^{*}$ AERMET and SHARP

\begin{tabular}{|c|c|c|c|c|c|c|c|c|c|c|c|c|}
\hline Rank & Conc. & YYMMDDHH & $\mathrm{u}^{*}$ & $\mathrm{w}^{*}$ & VPTG & $Z_{\text {ic }}$ & $z_{\text {im }}$ & $\mathbf{L}$ & $\mathrm{z}_{0}$ & WSPD & WDIR & temp \\
\hline 1 & 150.29 & 10030515 & 0.104 & 0.33 & 0.038 & 322 & 80 & -25 & 0.007 & 1.7 & 109.5 & 270.6 \\
\hline 2 & 142.14 & 8013022 & 0.124 & -9 & -9 & -999 & 106 & 11 & 0.007 & 3.66 & 127.6 & 252.7 \\
\hline 3 & 141.60 & 10021212 & 0.107 & 0.513 & 0.019 & 232 & 85 & -5.3 & 0.007 & 1.56 & 121.1 & 263.8 \\
\hline 4 & 112.60 & 7081308 & 0.228 & 0.576 & 0.021 & 177 & 262 & -27.6 & 0.105 & 2.23 & 104.4 & 293.8 \\
\hline 5 & 107.59 & 7031613 & 0.192 & 0.533 & 0.018 & 250 & 201 & -29.1 & 0.007 & 3.17 & 115.7 & 275.1 \\
\hline 6 & 94.74 & 8020215 & 0.156 & 0.427 & 0.029 & 200 & 147 & -24.2 & 0.007 & 2.55 & 124.9 & 266.9 \\
\hline 7 & 92.78 & 8020313 & 0.171 & 0.467 & 0.024 & 235 & 169 & -28.7 & 0.007 & 2.82 & 104.2 & 264.9 \\
\hline 8 & 88.18 & 9061609 & 0.327 & 0.738 & 0.011 & 341 & 449 & -74.2 & 0.105 & 3.44 & 116.4 & 291.8 \\
\hline 9 & 84.85 & 10022517 & 0.439 & 0.26 & 0.011 & 160 & 698 & -1939.5 & 0.011 & 7.46 & 308 & 268.5 \\
\hline 10 & 83.50 & 7110811 & 0.204 & 0.947 & 0.011 & 334 & 221 & -8.3 & 0.014 & 2.73 & 117.2 & 275.4 \\
\hline 11 & 80.89 & 8110914 & 0.254 & 0.825 & 0.026 & 367 & 308 & -26.9 & 0.014 & 3.75 & 112.2 & 261.5 \\
\hline 12 & 80.49 & 10103011 & 0.237 & 0.967 & 0.017 & 312 & 276 & -11.5 & 0.105 & 2.1 & 111.2 & 276.9 \\
\hline 13 & 79.11 & 9091509 & 0.216 & 0.714 & 0.023 & 205 & 241 & -14.2 & 0.105 & 1.97 & 117.8 & 295.1 \\
\hline 14 & 78.87 & 8032016 & 0.23 & 0.527 & 0.013 & 358 & 265 & -74.9 & 0.007 & 3.98 & 112.5 & 273.3 \\
\hline 15 & 78.74 & 7052017 & 0.212 & 0.573 & 0.026 & 380 & 241 & -48 & 0.047 & 2.59 & 115.2 & 287.8 \\
\hline 16 & 77.26 & 9032017 & 0.136 & 0.466 & 0.023 & 307 & 120 & -19 & 0.007 & 2.19 & 113.8 & 279.8 \\
\hline 17 & 75.75 & 10042210 & 0.265 & 0.829 & 0.008 & 215 & 327 & -17.5 & 0.047 & 2.99 & 107.6 & 291.3 \\
\hline 18 & 74.27 & 9102114 & 0.174 & 0.607 & 0.005 & 469 & 178 & -27.7 & 0.105 & 1.7 & 113.7 & 278.5 \\
\hline 19 & 74.00 & 8013101 & 0.081 & -9 & -9 & -999 & 56 & 5.7 & 0.007 & 2.95 & 142.2 & 252.3 \\
\hline 20 & 72.45 & 10080810 & 0.292 & 1.03 & 0.005 & 269 & 378 & -15.3 & 0.105 & 2.68 & 125.5 & 304 \\
\hline 21 & 72.45 & 9020812 & 0.19 & 0.234 & 0.027 & 36 & 198 & -48.3 & 0.007 & 3.22 & 107.9 & 271.6 \\
\hline 22 & 70.54 & 9061709 & 0.364 & 1.033 & 0.007 & 475 & 528 & -52.1 & 0.105 & 3.75 & 130.2 & 292 \\
\hline 23 & 70.34 & 9121914 & 0.127 & 0.153 & 0.023 & 238 & 112 & -346.7 & 0.007 & 2.28 & 133.1 & 267.4 \\
\hline 24 & 69.53 & 7081410 & 0.268 & 1.184 & 0.016 & 434 & 335 & -12.6 & 0.105 & 2.41 & 114.1 & 291.8 \\
\hline 25 & 68.53 & 9031615 & 0.138 & 0.6 & 0.019 & 184 & 123 & -5.6 & 0.007 & 2.01 & 111.3 & 279.6 \\
\hline
\end{tabular}


Table 25 Meteorological Conditions for Top 25 Modeled Concentrations at North Dakota Beulah Monitor Using Beta $u^{\star}$ AERMET and SHARP

\begin{tabular}{|c|c|c|c|c|c|c|c|c|c|c|c|c|}
\hline Rank & Conc. & YYMMDDHH & $\mathrm{u}^{*}$ & $\mathbf{w}^{*}$ & VPTG & $Z_{i c}$ & $z_{\text {im }}$ & $\mathbf{L}$ & $z_{0}$ & WSPD & WDIR & temp \\
\hline 1 & 128.34 & 8022212 & 0.204 & 0.36 & 0.018 & 76 & 221 & -34.2 & 0.007 & 3.4 & 96.7 & 272.5 \\
\hline 2 & 116.13 & 8070609 & 0.287 & 0.889 & 0.005 & 210 & 369 & -17.7 & 0.105 & 2.68 & 332.7 & 300.4 \\
\hline 3 & 114.87 & 10030516 & 0.124 & 0.218 & 0.038 & 322 & 105 & -148.4 & 0.007 & 2.19 & 88.6 & 270.5 \\
\hline 4 & 102.78 & 10022312 & 0.083 & 0.244 & 0.005 & 212 & 57 & -20.7 & 0.007 & 1.34 & 89.7 & 259 \\
\hline 5 & 97.16 & 10021516 & 0.138 & 0.32 & 0.03 & 183 & 124 & -37.3 & 0.007 & 2.32 & 324.9 & 261.4 \\
\hline 6 & 95.36 & 10022712 & 0.193 & 0.575 & 0.026 & 227 & 203 & -21.4 & 0.007 & 3.13 & 98.6 & 262.1 \\
\hline 7 & 93.44 & 10030214 & 0.067 & 0.323 & 0.02 & 249 & 42 & -5.6 & 0.007 & 0.98 & 83.2 & 267.3 \\
\hline 8 & 89.74 & 10020915 & 0.091 & 0.442 & 0.012 & 222 & 147 & -4.8 & 0.007 & 1.3 & 84.7 & 255.4 \\
\hline 9 & 89.32 & 8020314 & 0.173 & 0.494 & 0.024 & 277 & 172 & -29.6 & 0.007 & 2.86 & 97.1 & 265 \\
\hline 10 & 89.04 & 9021612 & 0.153 & 0.191 & 0.03 & 225 & 143 & -287.2 & 0.007 & 2.73 & 95.5 & 261.5 \\
\hline 11 & 87.53 & 7031614 & 0.245 & 0.551 & 0.017 & 278 & 291 & -61 & 0.007 & 4.2 & 92.8 & 275.6 \\
\hline 12 & 84.51 & 10051110 & 0.148 & 0.661 & 0.005 & 429 & 137 & -12.1 & 0.047 & 1.61 & 92.3 & 280.6 \\
\hline 13 & 84.46 & 8022315 & 0.174 & 0.42 & 0.013 & 112 & 174 & -20.1 & 0.007 & 2.82 & 91.8 & 278.2 \\
\hline 14 & 81.36 & 10021212 & 0.107 & 0.513 & 0.019 & 232 & 85 & -5.3 & 0.007 & 1.56 & 121.1 & 263.8 \\
\hline 15 & 79.99 & 10072410 & 0.212 & 0.784 & 0.006 & 231 & 235 & -11.4 & 0.105 & 1.88 & 359 & 293.6 \\
\hline 16 & 76.06 & 9020215 & 0.083 & 0.465 & 0.016 & 247 & 58 & -3.6 & 0.007 & 1.16 & 87.2 & 252.9 \\
\hline 17 & 74.84 & 10020315 & 0.074 & 0.345 & 0.027 & 151 & 48 & -3.7 & 0.007 & 1.03 & 127.4 & 266.2 \\
\hline 18 & 74.29 & 8051809 & 0.262 & 0.841 & 0.012 & 257 & 322 & -19.5 & 0.047 & 2.99 & 97 & 292.2 \\
\hline 19 & 73.20 & 8032013 & 0.255 & 0.427 & 0.014 & 337 & 308 & -179.1 & 0.007 & 4.51 & 96.9 & 272.5 \\
\hline 20 & 69.85 & 9021516 & 0.211 & 0.306 & 0.017 & 160 & 232 & -131.7 & 0.007 & 3.71 & 328.7 & 262.6 \\
\hline 21 & 69.40 & 10110814 & 0.106 & 0.813 & 0.015 & 224 & 83 & -1.2 & 0.014 & 1.12 & 169.7 & 287.9 \\
\hline 22 & 66.76 & 9031415 & 0.187 & 0.606 & 0.021 & 219 & 195 & -16.2 & 0.007 & 2.99 & 209.2 & 277.9 \\
\hline 23 & 65.80 & 8122514 & 0.21 & 0.076 & 0.013 & 17 & 232 & -874.4 & 0.007 & 3.8 & 100 & 260.1 \\
\hline 24 & 65.17 & 8030917 & 0.098 & 0.397 & 0.005 & 413 & 74 & -15.6 & 0.007 & 1.56 & 78.9 & 276.4 \\
\hline 25 & 63.35 & 8021516 & 0.146 & 0.322 & 0.031 & 174 & 136 & -40.7 & 0.007 & 2.46 & 225.5 & 276.8 \\
\hline
\end{tabular}


Gibson Meteorological

Conditions Resulting in Top 25 Concentrations 
This supplemental document presents meteorological conditions resulting in peak hourly $\mathrm{SO}_{2}$ observed and predicted concentration at the four Gibson monitors (show in the figure below).

- Table 1-4 show the meteorological conditions for the Gibson peak observed concentrations using beta $u^{*}$ AERMET.

- Table 5-8 show the meteorological conditions for the Gibson peak predicted concentrations with default AERMET and default AERMOD options (Test Case 1).

- Table 9-12 show the meteorological conditions for the Gibson peak predicted concentrations with beta $u^{*}$ AERMET and default AERMOD options, minimum sigma-v value of $0.2 \mathrm{~m} / \mathrm{s}$ (Test Case 2).

- Table 13- 16 show the meteorological conditions for the Gibson peak predicted concentrations with beta $u^{*}$ AERMET and beta AERMOD with minimum sigma- $v=0.5 \mathrm{~m} / \mathrm{s}$ options (Test Case 4).

- Table $17-20$ show the meteorological conditions for the Gibson peak predicted concentrations with beta $u^{*}$ AERMET and SHARP (Test Case 5).

In the tables below, the respective columns indicate ranked concentrations, year-month-day-hour, friction velocity $\left(u_{*}, \mathrm{~m} / \mathrm{s}\right)$, convective velocity scale $\left(w_{*}, \mathrm{~m} / \mathrm{s}\right)$, vertical potential temperature gradient at the top of the mixing lid (available if a convective, daytime hour - VPTG in deg $\mathrm{C} / \mathrm{m}$ ), convective mixing height (Zic in $\mathrm{m}$ ), mechanical mixing height (Zim in $\mathrm{m}$ ), Monin-Obukhov length ( $\mathrm{L}$, in $\mathrm{m}$ ), surface roughness length ( $\mathrm{zO}$, in $\mathrm{m})$, 10-m wind speed (WSPD, in $\mathrm{m} / \mathrm{s}$ ), 10-m wind direction (WDIR, in deg), and the surface air temperature in deg $\mathrm{K}$.

\section{Figure $1 \quad$ Map of Gibson Model Evaluation Layout}

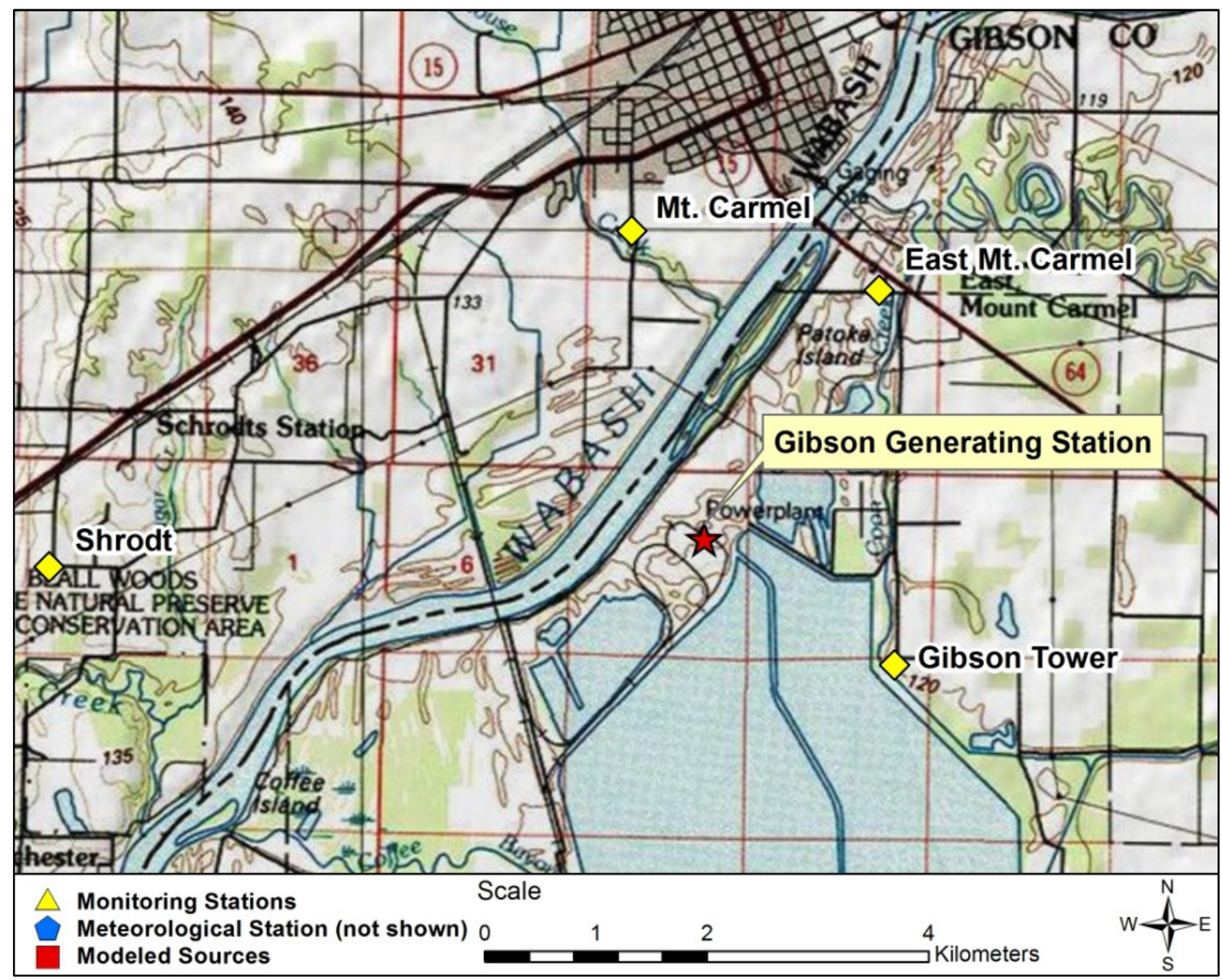


Table 1 Meteorological Conditions for Top 25 Observed Concentrations at Gibson Mt. Carmel Monitor Using Beta $u^{*}$ AERMET

\begin{tabular}{|c|c|c|c|c|c|c|c|c|c|c|c|c|}
\hline Rank & Conc. & YYMMDDHH & $\mathrm{u}^{*}$ & $\mathbf{w}^{*}$ & VPTG & $Z_{\text {ic }}$ & $z_{i m}$ & $\mathbf{L}$ & $\mathrm{z}_{0}$ & WSPD & WDIR & temp \\
\hline 1 & 544.41 & 10092012 & 0.19 & 2.144 & 0.006 & 1195 & 201 & -2.1 & 0.04 & 1.65 & 56 & 305.4 \\
\hline 2 & 326.12 & 10092013 & 0.238 & 2.205 & 0.005 & 1324 & 278 & -4.1 & 0.103 & 1.76 & 135 & 305.9 \\
\hline 3 & 323.49 & 10092010 & 0.284 & 1.501 & 0.005 & 513 & 362 & -8.6 & 0.066 & 2.66 & 156 & 302.0 \\
\hline 4 & 307.71 & 10092011 & 0.261 & 1.871 & 0.005 & 845 & 320 & -5.7 & 0.103 & 2.04 & 140 & 304.2 \\
\hline 5 & 291.93 & 9010917 & 0.269 & -9 & -9 & -999 & 336 & 118.6 & 0.035 & 3.78 & 154 & 282.0 \\
\hline 6 & 286.67 & 8120306 & 0.264 & -9 & -9 & -999 & 325 & 54.1 & 0.035 & 3.76 & 154 & 274.9 \\
\hline 7 & 284.04 & 8012819 & 0.467 & -9 & -9 & -999 & 766 & 348.4 & 0.035 & 6.42 & 163 & 282.0 \\
\hline 8 & 278.78 & 8010419 & 0.203 & -9 & -9 & -999 & 219 & 32.3 & 0.021 & 3.2 & 142 & 277.0 \\
\hline 9 & 249.85 & 10043006 & 0.244 & -9 & -9 & -999 & 290 & 73.9 & 0.032 & 3.52 & 145 & 289.9 \\
\hline 10 & 236.70 & 8120305 & 0.249 & -9 & -9 & -999 & 298 & 48.2 & 0.035 & 3.56 & 152 & 274.2 \\
\hline 11 & 236.70 & 8120821 & 0.337 & -9 & -9 & -999 & 470 & 90.9 & 0.035 & 4.76 & 161 & 282.0 \\
\hline 12 & 234.07 & 8060601 & 0.309 & -9 & -9 & -999 & 413 & 80.8 & 0.075 & 3.74 & 151 & 299.2 \\
\hline 13 & 226.18 & 10092014 & 0.214 & 2.198 & 0.005 & 1454 & 238 & -3.4 & 0.04 & 2.01 & 56 & 306.4 \\
\hline 14 & 210.40 & 8121307 & 0.221 & -9 & -9 & -999 & 250 & 37.7 & 0.035 & 3.18 & 156 & 271.4 \\
\hline 15 & 205.14 & 9021719 & 0.382 & -9 & -9 & -999 & 567 & 230.9 & 0.035 & 5.29 & 170 & 279.2 \\
\hline 16 & 199.88 & 8010515 & 0.273 & 0.455 & 0.015 & 299 & 342 & -160.7 & 0.035 & 3.59 & 152 & 280.4 \\
\hline 17 & 186.73 & 8012522 & 0.201 & -9 & -9 & -999 & 216 & 62.2 & 0.035 & 2.86 & 150 & 271.4 \\
\hline 18 & 184.10 & 8010418 & 0.201 & -9 & -9 & -999 & 228 & 31.7 & 0.021 & 3.17 & 147 & 277.0 \\
\hline 19 & 184.10 & 9022508 & 0.17 & 0.107 & 0.017 & 20 & 168 & -205.8 & 0.021 & 2.47 & 134 & 280.4 \\
\hline 20 & 181.47 & 9021106 & 0.529 & -9 & -9 & -999 & 924 & 460.3 & 0.035 & 7.25 & 168 & 289.9 \\
\hline 21 & 181.47 & 9051307 & 0.279 & 0.262 & 0.007 & 115 & 353 & -347.7 & 0.04 & 3.63 & 161 & 290.9 \\
\hline 22 & 178.84 & 9072012 & 0.228 & 1.409 & 0.011 & 1121 & 262 & -11.9 & 0.11 & 1.94 & 139 & 298.8 \\
\hline 23 & 176.21 & 10042920 & 0.335 & -9 & -9 & -999 & 466 & 93.6 & 0.04 & 4.61 & 162 & 294.2 \\
\hline 24 & 173.58 & 10051304 & 0.262 & -9 & -9 & -999 & 322 & 57.5 & 0.04 & 3.64 & 161 & 295.4 \\
\hline 25 & 170.95 & 9020920 & 0.398 & -9 & -9 & -999 & 602 & 149.2 & 0.035 & 5.56 & 167 & 290.9 \\
\hline
\end{tabular}

Table 2 Meteorological Conditions for Top 25 Observed Concentrations at Gibson East Mt. Carmel Using Beta $u^{*}$ AERMET

\begin{tabular}{|c|c|c|c|c|c|c|c|c|c|c|c|c|}
\hline Rank & Conc. & YYMMDDHH & $\mathrm{u}^{*}$ & $\mathbf{w}^{*}$ & VPTG & $Z_{i c}$ & $z_{\text {im }}$ & $\mathbf{L}$ & $z_{0}$ & WSPD & WDIR & temp \\
\hline 1 & 336.64 & 8120210 & 0.29 & 0.717 & 0.005 & 321 & 375 & -53.3 & 0.035 & 3.66 & 216 & 275.4 \\
\hline 2 & 331.38 & 8062821 & 0.405 & -9 & -9 & -999 & 618 & 276 & 0.052 & 5.17 & 201 & 297.5 \\
\hline 3 & 323.49 & 8051722 & 0.373 & -9 & -9 & -999 & 547 & 232.3 & 0.04 & 5.04 & 210 & 294.2 \\
\hline 4 & 323.49 & 10080311 & 0.384 & 2.068 & 0.005 & 1079 & 572 & -17.3 & 0.049 & 4.17 & 214 & 305.9 \\
\hline 5 & 294.56 & 10092012 & 0.19 & 2.144 & 0.006 & 1195 & 201 & -2.1 & 0.04 & 1.65 & 56 & 305.4 \\
\hline 6 & 270.89 & 8120211 & 0.263 & 0.94 & 0.006 & 497 & 325 & -27.4 & 0.015 & 3.75 & 195 & 275.9 \\
\hline 7 & 257.74 & 9080908 & 0.315 & 0.946 & 0.008 & 404 & 425 & -37.4 & 0.049 & 3.64 & 210 & 301.4 \\
\hline 8 & 249.85 & 10092011 & 0.261 & 1.871 & 0.005 & 845 & 320 & -5.7 & 0.103 & 2.04 & 140 & 304.2 \\
\hline 9 & 247.22 & 10011105 & 0.344 & -9 & -9 & -999 & 484 & 179.5 & 0.015 & 5.53 & 207 & 268.1 \\
\hline 10 & 241.96 & 10011106 & 0.295 & -9 & -9 & -999 & 387 & 66.4 & 0.015 & 4.85 & 208 & 268.8 \\
\hline 11 & 236.70 & 8062822 & 0.542 & -9 & -9 & -999 & 958 & 282.9 & 0.049 & 7.01 & 213 & 297.0 \\
\hline 12 & 236.70 & 8070209 & 0.489 & -9 & -9 & -999 & 820 & -114.4 & 0.049 & 5.96 & 210 & 300.4 \\
\hline 13 & 234.07 & 8011811 & 0.312 & 1.087 & 0.006 & 365 & 418 & -21.5 & 0.015 & 4.37 & 197 & 270.9 \\
\hline 14 & 226.18 & 9080910 & 0.409 & 1.264 & 0.005 & 490 & 627 & -41.4 & 0.049 & 4.75 & 215 & 304.2 \\
\hline 15 & 223.55 & 9080909 & 0.336 & 1.128 & 0.006 & 444 & 467 & -29.3 & 0.049 & 3.81 & 216 & 303.1 \\
\hline 16 & 213.03 & 9121112 & 0.228 & 1.009 & 0.007 & 414 & 261 & -11.9 & 0.015 & 3.05 & 200 & 275.4 \\
\hline 17 & 210.40 & 8021715 & 0.732 & 0.599 & 0.008 & 501 & 1502 & -2293.5 & 0.035 & 9.9 & 217 & 287.5 \\
\hline 18 & 194.62 & 10072209 & 0.276 & 0.918 & 0.006 & 435 & 349 & -29.7 & 0.052 & 3.1 & 206 & 302.5 \\
\hline 19 & 191.99 & 9042414 & 0.777 & 1.89 & 0.009 & 1727 & 1644 & -299.2 & 0.04 & 10.09 & 214 & 303.1 \\
\hline 20 & 189.36 & 8060905 & 0.331 & -9 & -9 & -999 & 456 & 92.2 & 0.052 & 4.32 & 197 & 297.5 \\
\hline 21 & 184.10 & 10062716 & 0.687 & 1.866 & 0.005 & 1862 & 1365 & -232.3 & 0.049 & 8.54 & 223 & 306.4 \\
\hline 22 & 181.47 & 8120610 & 0.433 & 0.305 & 0.007 & 145 & 684 & -1044.7 & 0.015 & 6.75 & 208 & 273.1 \\
\hline 23 & 181.47 & 10051309 & 0.498 & 1.307 & 0.006 & 760 & 842 & -104.9 & 0.04 & 6.3 & 212 & 299.9 \\
\hline 24 & 178.84 & 10072711 & 0.286 & 1.559 & 0.005 & 783 & 368 & -12.1 & 0.049 & 3 & 228 & 303.1 \\
\hline 25 & 173.58 & 8012713 & 0.375 & 1.283 & 0.01 & 459 & 552 & -28.7 & 0.015 & 5.36 & 206 & 279.2 \\
\hline
\end{tabular}


Table 3 Meteorological Conditions for Top 25 Observed Concentrations at Gibson Shrodt Monitor Using Beta $u^{*}$ AERMET

\begin{tabular}{|c|c|c|c|c|c|c|c|c|c|c|c|c|}
\hline Rank & Conc. & YYMMDDHH & $\mathrm{u}^{*}$ & $\mathbf{w}^{*}$ & VPTG & $z_{\text {ic }}$ & $z_{\text {im }}$ & $\mathbf{L}$ & $\mathrm{z}_{0}$ & WSPD & WDIR & temp \\
\hline 1 & 236.70 & 9030316 & 0.202 & 0.995 & 0.018 & 770 & 219 & -16.3 & 0.032 & 2.4 & 124 & 273.1 \\
\hline 2 & 197.25 & 8081109 & 0.215 & 1.764 & 0.013 & 1043 & 240 & -4.7 & 0.047 & 2.03 & 55 & 294.2 \\
\hline 3 & 189.36 & 8091810 & 0.309 & 1.74 & 0.005 & 807 & 413 & -11.3 & 0.04 & 3.37 & 58 & 299.2 \\
\hline 4 & 189.36 & 10052909 & 0.243 & 1.459 & 0.005 & 516 & 288 & -6 & 0.038 & 2.5 & 69 & 300.4 \\
\hline 5 & 176.21 & 8092114 & 0.271 & 2.439 & 0.005 & 2088 & 338 & -7.1 & 0.04 & 2.81 & 41 & 299.9 \\
\hline 6 & 165.69 & 10081810 & 0.334 & 1.789 & 0.005 & 846 & 463 & -13.7 & 0.124 & 2.79 & 118 & 301.4 \\
\hline 7 & 160.43 & 10050613 & 0.207 & 2.212 & 0.012 & 1333 & 227 & -2.7 & 0.022 & 2.18 & 36 & 297.5 \\
\hline 8 & 157.80 & 10050614 & 0.256 & 2.18 & 0.011 & 1382 & 312 & -5.6 & 0.038 & 2.61 & 75 & 297.0 \\
\hline 9 & 155.17 & 10051612 & 0.26 & 1.243 & 0.009 & 937 & 320 & -21.5 & 0.038 & 3.05 & 83 & 292.0 \\
\hline 10 & 149.91 & 8072709 & 0.184 & 0.976 & 0.005 & 386 & 189 & -6.5 & 0.047 & 1.81 & 52 & 300.9 \\
\hline 11 & 147.28 & 8122511 & 0.222 & 0.777 & 0.028 & 332 & 251 & -19.4 & 0.026 & 2.79 & 88 & 271.4 \\
\hline 12 & 144.65 & 8083110 & 0.386 & 1.9 & 0.007 & 1066 & 576 & -22.3 & 0.119 & 3.44 & 73 & 303.8 \\
\hline 13 & 144.65 & 9032008 & 0.245 & 0.438 & 0.018 & 123 & 292 & -54.2 & 0.038 & 3.05 & 65 & 276.4 \\
\hline 14 & 144.65 & 9111210 & 0.192 & 0.883 & 0.017 & 193 & 203 & -5 & 0.015 & 2.37 & 52 & 283.8 \\
\hline 15 & 139.39 & 9111511 & 0.098 & 0.821 & 0.011 & 509 & 74 & -2.2 & 0.035 & 0.89 & 222 & 290.9 \\
\hline 16 & 136.76 & 9092611 & 0.217 & 1.134 & 0.006 & 449 & 243 & -7.9 & 0.117 & 1.71 & 92 & 294.9 \\
\hline 17 & 134.13 & 10082810 & 0.27 & 1.971 & 0.012 & 1179 & 338 & -7.5 & 0.124 & 2.08 & 113 & 301.4 \\
\hline 18 & 131.50 & 8011615 & 0.242 & 0.92 & 0.011 & 354 & 286 & -16.2 & 0.026 & 3 & 118 & 275.9 \\
\hline 19 & 131.50 & 9100509 & 0.256 & 0.612 & 0.005 & 127 & 311 & -23.2 & 0.117 & 2.3 & 99 & 287.5 \\
\hline 20 & 128.87 & 10051609 & 0.269 & 0.946 & 0.005 & 302 & 335 & -17.4 & 0.022 & 3.46 & 58 & 289.9 \\
\hline 21 & 126.24 & 9082712 & 0.171 & 1.542 & 0.005 & 1126 & 170 & -3.8 & 0.052 & 1.53 & 1 & 302.0 \\
\hline 22 & 123.61 & 9090312 & 0.192 & 1.659 & 0.005 & 1120 & 202 & -4.3 & 0.045 & 1.81 & 19 & 298.8 \\
\hline 23 & 120.98 & 8091811 & 0.334 & 2.114 & 0.007 & 1230 & 462 & -12.1 & 0.117 & 2.79 & 91 & 301.4 \\
\hline 24 & 120.98 & 9051808 & 0.242 & 0.572 & 0.005 & 127 & 286 & -24.2 & 0.038 & 2.86 & 69 & 287.5 \\
\hline 25 & 120.98 & 10030611 & 0.202 & 0.766 & 0.009 & 231 & 218 & -10.6 & 0.038 & 2.21 & 102 & 281.4 \\
\hline
\end{tabular}

Table 4 Meteorological Conditions for Top 25 Observed Concentrations at Gibson Tower Monitor Using Beta $u^{*}$ AERMET

\begin{tabular}{|c|c|c|c|c|c|c|c|c|c|c|c|c|}
\hline Rank & Conc. & YYMMDDHH & $\mathrm{u}^{*}$ & $\mathrm{w}^{*}$ & VPTG & $Z_{\text {ic }}$ & $z_{\text {im }}$ & $\mathbf{L}$ & $z_{0}$ & WSPD & WDIR & temp \\
\hline 1 & 355.05 & 9072011 & 0.178 & 1.447 & 0.005 & 877 & 181 & -4.1 & 0.047 & 1.65 & 37 & 298.1 \\
\hline 2 & 181.47 & 8072712 & 0.172 & 1.514 & 0.013 & 944 & 171 & -3.5 & 0.032 & 1.71 & 281 & 303.1 \\
\hline 3 & 178.84 & 8011217 & 0.135 & -9 & -9 & -999 & 123 & 18.2 & 0.026 & 2.1 & 116 & 280.9 \\
\hline 4 & 178.84 & 8011612 & 0.287 & 1.07 & 0.01 & 298 & 369 & -14.4 & 0.026 & 3.51 & 110 & 274.9 \\
\hline 5 & 173.58 & 9062913 & 0.641 & 2.637 & 0.01 & 2038 & 1230 & -72.9 & 0.032 & 8.35 & 281 & 304.2 \\
\hline 6 & 147.28 & 8061414 & 0.262 & 2.009 & 0.005 & 1648 & 323 & -9.2 & 0.049 & 2.67 & 212 & 300.9 \\
\hline 7 & 147.28 & 10091916 & 0.232 & 1.82 & 0.005 & 1493 & 268 & -7.7 & 0.045 & 2.36 & 14 & 303.1 \\
\hline 8 & 144.65 & 9062912 & 0.614 & 2.615 & 0.011 & 1981 & 1154 & -64 & 0.032 & 7.96 & 279 & 303.8 \\
\hline 9 & 139.39 & 10062517 & 0.296 & 1.576 & 0.009 & 1681 & 387 & -27.9 & 0.079 & 3 & 331 & 304.9 \\
\hline 10 & 136.76 & 10062516 & 0.258 & 1.781 & 0.008 & 1648 & 315 & -12.5 & 0.047 & 2.74 & 323 & 305.4 \\
\hline 11 & 131.50 & 8061413 & 0.215 & 2.037 & 0.005 & 1607 & 240 & -4.7 & 0.049 & 2.01 & 229 & 301.4 \\
\hline 12 & 128.87 & 8102814 & 0.428 & 1.869 & 0.017 & 1493 & 673 & -44.8 & 0.027 & 5.63 & 285 & 282.0 \\
\hline 13 & 120.98 & 10091116 & 0.49 & 1.706 & 0.011 & 1148 & 822 & -67.8 & 0.041 & 6.06 & 301 & 300.9 \\
\hline 14 & 118.35 & 8011611 & 0.248 & 0.963 & 0.019 & 257 & 297 & -11 & 0.026 & 2.96 & 99 & 273.8 \\
\hline 15 & 118.35 & 10040910 & 0.348 & 1.446 & 0.005 & 968 & 492 & -33.5 & 0.026 & 4.53 & 311 & 287.0 \\
\hline 16 & 118.35 & 10062515 & 0.253 & 1.9 & 0.008 & 1594 & 305 & -9.4 & 0.041 & 2.69 & 258 & 304.9 \\
\hline 17 & 115.72 & 9063013 & 0.446 & 2.494 & 0.008 & 1736 & 716 & -24.7 & 0.032 & 5.46 & 284 & 301.4 \\
\hline 18 & 115.72 & 9072610 & 0.262 & 1.292 & 0.005 & 709 & 321 & -14.7 & 0.041 & 2.91 & 267 & 300.9 \\
\hline 19 & 115.72 & 10040914 & 0.278 & 1.719 & 0.014 & 1429 & 353 & -15.1 & 0.026 & 3.42 & 319 & 289.9 \\
\hline 20 & 115.72 & 10090314 & 0.532 & 2.433 & 0.008 & 1759 & 932 & -46 & 0.027 & 7.01 & 298 & 299.9 \\
\hline 21 & 113.09 & 8011614 & 0.238 & 1.058 & 0.013 & 342 & 280 & -9.8 & 0.026 & 2.81 & 101 & 275.9 \\
\hline 22 & 113.09 & 10022612 & 0.413 & 1.712 & 0.011 & 801 & 637 & -28.1 & 0.018 & 5.7 & 298 & 275.9 \\
\hline 23 & 113.09 & 10050801 & 0.485 & -9 & -9 & -999 & 813 & 190.9 & 0.025 & 7.16 & 299 & 286.4 \\
\hline 24 & 110.46 & 10040911 & 0.373 & 1.597 & 0.011 & 1114 & 545 & -35.3 & 0.026 & 4.87 & 317 & 287.5 \\
\hline 25 & 107.83 & 10050813 & 0.496 & 1.759 & 0.013 & 1400 & 839 & -78.3 & 0.025 & 6.79 & 299 & 287.5 \\
\hline
\end{tabular}


Table 5 Meteorological Conditions for Top 25 Modeled Concentrations at Gibson Mt. Carmel Monitor Using Default AERMET and AERMOD

\begin{tabular}{|c|c|c|c|c|c|c|c|c|c|c|c|c|}
\hline Rank & Conc. & YYMMDDHH & $\mathrm{u}^{*}$ & $\mathbf{w}^{*}$ & VPTG & $Z_{\text {ic }}$ & $z_{\text {im }}$ & $\mathbf{L}$ & $z_{0}$ & WSPD & WDIR & temp \\
\hline 1 & 518.86 & 10062210 & 0.293 & 1.296 & 0.005 & 487 & 381 & -14.1 & 0.075 & 2.81 & 155 & 303.8 \\
\hline 2 & 399.42 & 9121612 & 0.156 & 0.858 & 0.024 & 261 & 148 & -4 & 0.035 & 1.55 & 166 & 272.0 \\
\hline 3 & 338.67 & 8111009 & 0.091 & 0.725 & 0.016 & 300 & 66 & -1.5 & 0.015 & 0.96 & 180 & 275.9 \\
\hline 4 & 336.22 & 8110210 & 0.137 & 0.84 & 0.01 & 218 & 122 & -2.4 & 0.035 & 1.26 & 173 & 292.5 \\
\hline 5 & 326.57 & 9080319 & 0.269 & -9 & -9 & -999 & 337 & 83.6 & 0.075 & 3.46 & 172 & 300.9 \\
\hline 6 & 322.20 & 10071720 & 0.16 & -9 & -9 & -999 & 165 & 22.9 & 0.075 & 2.56 & 170 & 301.4 \\
\hline 7 & 317.40 & 10080319 & 0.192 & -9 & -9 & -999 & 208 & 47.4 & 0.075 & 2.64 & 173 & 307.0 \\
\hline 8 & 293.70 & 8121814 & 0.096 & 0.359 & 0.023 & 191 & 72 & -9.2 & 0.035 & 1.06 & 175 & 274.2 \\
\hline 9 & 286.17 & 9102109 & 0.198 & 0.681 & 0.013 & 222 & 212 & -13.7 & 0.066 & 1.96 & 176 & 287.0 \\
\hline 10 & 275.44 & 9102611 & 0.2 & 0.934 & 0.005 & 313 & 214 & -7.6 & 0.066 & 1.84 & 163 & 290.9 \\
\hline 11 & 272.86 & 9080514 & 0.124 & 0.732 & 0.006 & 328 & 106 & -4 & 0.075 & 1.01 & 165 & 298.8 \\
\hline 12 & 266.85 & 8112324 & 0.206 & -9 & -9 & -999 & 225 & 67 & 0.035 & 3.1 & 172 & 278.8 \\
\hline 13 & 265.32 & 8122619 & 0.348 & -9 & -9 & -999 & 493 & 168.1 & 0.035 & 4.93 & 170 & 290.9 \\
\hline 14 & 264.04 & 8060220 & 0.136 & -9 & -9 & -999 & 122 & 15.8 & 0.075 & 2.45 & 171 & 298.8 \\
\hline 15 & 261.48 & 8020501 & 0.39 & -9 & -9 & -999 & 585 & 125.7 & 0.035 & 5.6 & 172 & 291.4 \\
\hline 16 & 259.21 & 9021719 & 0.378 & -9 & -9 & -999 & 557 & 225.8 & 0.035 & 5.29 & 170 & 279.2 \\
\hline 17 & 254.80 & 8010604 & 0.347 & -9 & -9 & -999 & 490 & 194.8 & 0.035 & 4.88 & 172 & 285.9 \\
\hline 18 & 254.66 & 8120822 & 0.405 & -9 & -9 & -999 & 619 & 131.4 & 0.035 & 5.8 & 170 & 282.5 \\
\hline 19 & 254.40 & 8061320 & 0.158 & -9 & -9 & -999 & 237 & 21.8 & 0.075 & 2.56 & 172 & 294.9 \\
\hline 20 & 253.12 & 8120219 & 0.253 & -9 & -9 & -999 & 305 & 50.1 & 0.035 & 3.93 & 172 & 276.4 \\
\hline 21 & 252.49 & 8121321 & 0.422 & -9 & -9 & -999 & 658 & 140.9 & 0.035 & 6.02 & 172 & 279.2 \\
\hline 22 & 251.84 & 8020417 & 0.336 & -9 & -9 & -999 & 469 & 356.5 & 0.035 & 4.65 & 170 & 292.5 \\
\hline 23 & 248.20 & 8102421 & 0.22 & -9 & -9 & -999 & 248 & 77.9 & 0.066 & 2.91 & 171 & 285.4 \\
\hline 24 & 239.08 & 10033121 & 0.3 & -9 & -9 & -999 & 393 & 74.6 & 0.04 & 4.36 & 171 & 293.8 \\
\hline 25 & 237.87 & 10051220 & 0.151 & -9 & -9 & -999 & 144 & 19.2 & 0.04 & 2.78 & 172 & 297.0 \\
\hline
\end{tabular}

Table 6 Meteorological Conditions for Top 25 Modeled Concentrations at Gibson East Mt. Carmel Monitor Using Default AERMET and AERMOD

\begin{tabular}{|c|c|c|c|c|c|c|c|c|c|c|c|c|}
\hline Rank & Conc. & YYMMDDHH & $\mathrm{u}^{*}$ & $\mathrm{w}^{*}$ & VPTG & $z_{i c}$ & $z_{i m}$ & $\mathbf{L}$ & $z_{0}$ & WSPD & WDIR & temp \\
\hline 1 & 310.53 & 9061920 & 0.364 & -9 & -9 & -999 & 535 & 113.4 & 0.049 & 4.95 & 219 & 302.0 \\
\hline 2 & 282.35 & 10011012 & 0.156 & 0.809 & 0.018 & 209 & 147 & -3.7 & 0.035 & 1.53 & 222 & 265.4 \\
\hline 3 & 264.41 & 10061320 & 0.086 & -9 & -9 & -999 & 131 & 7 & 0.049 & 2.18 & 218 & 302.0 \\
\hline 4 & 256.70 & 9061108 & 0.198 & 0.816 & 0.012 & 244 & 220 & -8.8 & 0.049 & 2.01 & 220 & 294.9 \\
\hline 5 & 250.44 & 9102310 & 0.604 & 0.653 & 0.006 & 249 & 1126 & -493.7 & 0.044 & 7.76 & 217 & 292.5 \\
\hline 6 & 246.68 & 10041310 & 0.219 & 0.995 & 0.005 & 301 & 246 & -8 & 0.04 & 2.3 & 216 & 297.5 \\
\hline 7 & 241.67 & 10062123 & 0.069 & -9 & -9 & -999 & 44 & 5.6 & 0.049 & 1.76 & 218 & 299.9 \\
\hline 8 & 236.25 & 10021111 & 0.147 & 1.118 & 0.014 & 302 & 136 & -1.7 & 0.035 & 1.28 & 231 & 269.9 \\
\hline 9 & 229.45 & 8052209 & 0.097 & 0.729 & 0.005 & 252 & 73 & -1.5 & 0.04 & 0.8 & 228 & 290.4 \\
\hline 10 & 228.60 & 10082919 & 0.314 & -9 & -9 & -999 & 429 & 83.7 & 0.049 & 4.37 & 219 & 299.2 \\
\hline 11 & 228.12 & 8051718 & 0.377 & 0.673 & 0.005 & 2016 & 563 & -883.8 & 0.04 & 4.95 & 220 & 296.4 \\
\hline 12 & 218.55 & 10081008 & 0.178 & 0.988 & 0.005 & 266 & 180 & -3.9 & 0.049 & 1.62 & 230 & 304.2 \\
\hline 13 & 218.27 & 10090221 & 0.082 & -9 & -9 & -999 & 56 & 6.6 & 0.044 & 2.13 & 219 & 296.4 \\
\hline 14 & 218.10 & 10040111 & 0.548 & 1.052 & 0.006 & 326 & 974 & -115.3 & 0.04 & 6.96 & 215 & 297.0 \\
\hline 15 & 215.32 & 10060524 & 0.405 & -9 & -9 & -999 & 618 & 138.8 & 0.049 & 5.44 & 217 & 298.8 \\
\hline 16 & 212.93 & 9090711 & 0.203 & 1.094 & 0.005 & 348 & 219 & -5.5 & 0.044 & 1.99 & 220 & 297.0 \\
\hline 17 & 212.55 & 10072101 & 0.313 & -9 & -9 & -999 & 421 & 95.2 & 0.049 & 4.31 & 218 & 299.9 \\
\hline 18 & 211.49 & 10050518 & 0.429 & 1.219 & 0.009 & 2467 & 676 & -268 & 0.04 & 5.56 & 215 & 299.2 \\
\hline 19 & 206.43 & 10041111 & 0.226 & 1.122 & 0.005 & 378 & 258 & -7.7 & 0.04 & 2.37 & 214 & 295.9 \\
\hline 20 & 205.37 & 10040721 & 0.453 & -9 & -9 & -999 & 730 & 335.7 & 0.04 & 6.12 & 217 & 289.2 \\
\hline 21 & 201.95 & 10062624 & 0.265 & -9 & -9 & -999 & 328 & 59.6 & 0.049 & 3.81 & 219 & 299.9 \\
\hline 22 & 201.72 & 9080918 & 0.407 & 1.058 & 0.005 & 1769 & 626 & -252 & 0.049 & 5.07 & 220 & 303.8 \\
\hline 23 & 200.59 & 10072018 & 0.407 & 1.056 & 0.006 & 1197 & 625 & -170.9 & 0.049 & 5.02 & 223 & 303.1 \\
\hline 24 & 200.27 & 10051711 & 0.237 & 0.947 & 0.008 & 430 & 277 & -16.9 & 0.04 & 2.69 & 215 & 292.5 \\
\hline 25 & 197.89 & 9061619 & 0.405 & -9 & -9 & -999 & 628 & 296.9 & 0.049 & 5.29 & 218 & 295.9 \\
\hline
\end{tabular}


Table 7 Meteorological Conditions for Top 25 Modeled Concentrations at Gibson Shrodt Monitor Using Default AERMET and Default AERMOD

\begin{tabular}{|c|c|c|c|c|c|c|c|c|c|c|c|c|}
\hline Rank & Conc. & YYMMDDHH & $\mathrm{u}^{*}$ & $\mathbf{w}^{*}$ & VPTG & $z_{\text {ic }}$ & $z_{i m}$ & $\mathbf{L}$ & $\mathrm{z}_{0}$ & WSPD & WDIR & temp \\
\hline 1 & 283.02 & 9102709 & 0.133 & 0.527 & 0.012 & 222 & 116 & -8.9 & 0.112 & 1.08 & 87 & 285.4 \\
\hline 2 & 280.21 & 10022110 & 0.119 & 0.583 & 0.012 & 231 & 98 & -4.9 & 0.026 & 1.3 & 87 & 281.4 \\
\hline 3 & 270.44 & 9042907 & 0.096 & 0.402 & 0.012 & 161 & 71 & -5.4 & 0.038 & 0.97 & 90 & 291.4 \\
\hline 4 & 244.03 & 9121610 & 0.161 & 0.624 & 0.029 & 177 & 156 & -7.7 & 0.026 & 1.86 & 92 & 268.8 \\
\hline 5 & 220.58 & 9121814 & 0.127 & 0.529 & 0.009 & 381 & 109 & -13.3 & 0.026 & 1.55 & 91 & 282.0 \\
\hline 6 & 207.35 & 8122511 & 0.222 & 0.777 & 0.028 & 332 & 251 & -19.4 & 0.026 & 2.79 & 88 & 271.4 \\
\hline 7 & 197.55 & 9050812 & 0.188 & 0.732 & 0.005 & 500 & 196 & -21.2 & 0.038 & 2.2 & 90 & 293.8 \\
\hline 8 & 184.82 & 10041210 & 0.187 & 0.937 & 0.009 & 255 & 194 & -5.1 & 0.038 & 1.88 & 96 & 294.9 \\
\hline 9 & 178.54 & 8021615 & 0.162 & 0.414 & 0.009 & 195 & 157 & -29.4 & 0.026 & 2.1 & 85 & 275.9 \\
\hline 10 & 172.89 & 9122311 & 0.239 & 0.843 & 0.013 & 273 & 281 & -15.6 & 0.026 & 2.95 & 97 & 282.0 \\
\hline 11 & 161.54 & 10012014 & 0.207 & 0.553 & 0.027 & 327 & 227 & -43.1 & 0.026 & 2.74 & 86 & 280.4 \\
\hline 12 & 158.31 & 9070907 & 0.178 & 0.565 & 0.009 & 215 & 180 & -16.7 & 0.124 & 1.52 & 91 & 294.9 \\
\hline 13 & 158.30 & 9080513 & 0.174 & 0.734 & 0.007 & 311 & 174 & -10.4 & 0.119 & 1.42 & 88 & 298.1 \\
\hline 14 & 155.64 & 8073010 & 0.243 & 0.943 & 0.005 & 272 & 288 & -11.6 & 0.124 & 1.99 & 96 & 301.4 \\
\hline 15 & 154.74 & 10021415 & 0.164 & 0.66 & 0.007 & 375 & 160 & -14.4 & 0.026 & 2.01 & 90 & 273.1 \\
\hline 16 & 143.48 & 10032514 & 0.265 & 0.56 & 0.011 & 316 & 327 & -83.7 & 0.038 & 3.36 & 86 & 285.4 \\
\hline 17 & 139.88 & 9021311 & 0.216 & 1.131 & 0.005 & 443 & 241 & -7.7 & 0.026 & 2.49 & 89 & 279.2 \\
\hline 18 & 139.58 & 8090407 & 0.23 & 0.486 & 0.015 & 186 & 264 & -49.2 & 0.117 & 2.2 & 93 & 295.9 \\
\hline 19 & 137.53 & 8092008 & 0.18 & 0.822 & 0.008 & 222 & 183 & -5.9 & 0.112 & 1.38 & 81 & 294.9 \\
\hline 20 & 136.81 & 10020413 & 0.279 & 1.331 & 0.015 & 472 & 354 & -10.8 & 0.026 & 3.32 & 88 & 276.4 \\
\hline 21 & 131.98 & 10020809 & 0.193 & 0.507 & 0.007 & 198 & 204 & -27.5 & 0.026 & 2.49 & 85 & 269.9 \\
\hline 22 & 129.32 & 9082807 & 0.097 & 0.418 & 0.013 & 164 & 72 & -5.1 & 0.124 & 0.7 & 100 & 295.4 \\
\hline 23 & 124.35 & 8013114 & 0.164 & 0.609 & 0.011 & 256 & 167 & -12.5 & 0.026 & 1.98 & 78 & 275.4 \\
\hline 24 & 123.73 & 10061009 & 0.234 & 1.084 & 0.005 & 349 & 272 & -8.8 & 0.124 & 1.84 & 93 & 300.9 \\
\hline 25 & 122.46 & 9092411 & 0.183 & 0.551 & 0.005 & 196 & 188 & -17.9 & 0.117 & 1.6 & 91 & 296.4 \\
\hline
\end{tabular}

Table 8 Meteorological Conditions for Top 25 Modeled Concentrations at Gibson Tower Monitor Using Default AERMET and Default AERMOD

\begin{tabular}{|c|c|c|c|c|c|c|c|c|c|c|c|c|}
\hline Rank & Conc. & YYMMDDHH & $\mathrm{u}^{*}$ & $\mathbf{w}^{*}$ & VPTG & $z_{i c}$ & $z_{\text {im }}$ & L & $z_{0}$ & WSPD & WDIR & temp \\
\hline 1 & 440.35 & 9122113 & 0.069 & 0.544 & 0.005 & 337 & 44 & -1.8 & 0.018 & 0.72 & 295 & 274.9 \\
\hline 2 & 261.77 & 8112811 & 0.143 & 0.967 & 0.013 & 365 & 130 & -3 & 0.019 & 1.57 & 304 & 280.4 \\
\hline 3 & 243.51 & 9060207 & 0.135 & 0.766 & 0.016 & 199 & 120 & -2.8 & 0.047 & 1.18 & 304 & 297.5 \\
\hline 4 & 241.16 & 8081308 & 0.138 & 1.017 & 0.005 & 312 & 123 & -2 & 0.032 & 1.26 & 297 & 295.9 \\
\hline 5 & 237.06 & 9101210 & 0.11 & 0.834 & 0.01 & 242 & 87 & -1.4 & 0.027 & 0.99 & 293 & 288.1 \\
\hline 6 & 225.77 & 8070109 & 0.167 & 0.887 & 0.005 & 274 & 164 & -4.6 & 0.047 & 1.57 & 308 & 299.2 \\
\hline 7 & 216.48 & 10021911 & 0.139 & 1.216 & 0.01 & 351 & 124 & -1.3 & 0.018 & 1.37 & 278 & 277.0 \\
\hline 8 & 215.38 & 10050408 & 0.131 & 1.082 & 0.005 & 349 & 114 & -1.5 & 0.026 & 1.21 & 301 & 292.5 \\
\hline 9 & 185.94 & 10022612 & 0.413 & 1.712 & 0.011 & 801 & 637 & -28.1 & 0.018 & 5.7 & 298 & 275.9 \\
\hline 10 & 180.42 & 10091908 & 0.129 & 0.844 & 0.013 & 233 & 112 & -2.1 & 0.027 & 1.25 & 292 & 294.9 \\
\hline 11 & 179.89 & 9072309 & 0.205 & 1.006 & 0.005 & 422 & 223 & -8.9 & 0.047 & 2.1 & 308 & 297.0 \\
\hline 12 & 179.29 & 10081416 & 0.364 & 2.119 & 0.005 & 1790 & 527 & -22.7 & 0.047 & 4.09 & 303 & 309.9 \\
\hline 13 & 173.47 & 9070309 & 0.176 & 0.714 & 0.005 & 285 & 178 & -10.7 & 0.047 & 1.84 & 301 & 297.0 \\
\hline 14 & 171.69 & 10022512 & 0.258 & 1.085 & 0.011 & 302 & 314 & -10.1 & 0.019 & 3.25 & 309 & 272.5 \\
\hline 15 & 166.82 & 8100111 & 0.406 & 1.809 & 0.009 & 873 & 621 & -24.7 & 0.041 & 4.73 & 300 & 290.9 \\
\hline 16 & 166.45 & 10070909 & 0.221 & 0.811 & 0.005 & 299 & 249 & -15.1 & 0.047 & 2.39 & 306 & 298.8 \\
\hline 17 & 159.40 & 9062014 & 0.309 & 1.889 & 0.005 & 1573 & 412 & -17.1 & 0.032 & 3.68 & 298 & 305.4 \\
\hline 18 & 156.25 & 9061717 & 0.29 & 1.936 & 0.005 & 1762 & 376 & -14.8 & 0.032 & 3.41 & 298 & 305.9 \\
\hline 19 & 156.10 & 10041912 & 0.184 & 1.328 & 0.005 & 571 & 189 & -3.8 & 0.025 & 1.96 & 296 & 290.9 \\
\hline 20 & 153.22 & 10072909 & 0.241 & 1.108 & 0.005 & 393 & 283 & -10.1 & 0.047 & 2.5 & 310 & 302.5 \\
\hline 21 & 152.38 & 8061908 & 0.118 & 0.838 & 0.008 & 232 & 97 & -1.6 & 0.047 & 0.94 & 306 & 296.4 \\
\hline 22 & 146.04 & 8072110 & 0.224 & 1.187 & 0.005 & 536 & 254 & -9 & 0.032 & 2.5 & 293 & 305.9 \\
\hline 23 & 143.80 & 10062113 & 0.257 & 1.802 & 0.005 & 1146 & 317 & -8.3 & 0.032 & 2.86 & 298 & 307.0 \\
\hline 24 & 143.78 & 9073117 & 0.297 & 1.362 & 0.005 & 1627 & 389 & -42.3 & 0.047 & 3.49 & 302 & 299.2 \\
\hline 25 & 143.07 & 9050410 & 0.121 & 0.959 & 0.005 & 354 & 100 & -1.8 & 0.026 & 1.14 & 321 & 289.9 \\
\hline
\end{tabular}


Table 9 Meteorological Conditions for Top 25 Modeled Concentrations at Gibson Mt. Carmel Monitor Using Beta $\mathbf{u}^{\star}$ AERMET and Default AERMOD

\begin{tabular}{|c|c|c|c|c|c|c|c|c|c|c|c|c|}
\hline Rank & Conc. & YYMMDDHH & $u^{*}$ & $\mathbf{w}^{*}$ & VPTG & $Z_{\text {ic }}$ & $z_{\text {im }}$ & $\mathbf{L}$ & $z_{0}$ & WSPD & WDIR & temp \\
\hline 1 & 518.86 & 10062210 & 0.293 & 1.296 & 0.005 & 487 & 381 & -14.1 & 0.075 & 2.81 & 155 & 303.8 \\
\hline 2 & 399.42 & 9121612 & 0.156 & 0.858 & 0.024 & 261 & 148 & -4 & 0.035 & 1.55 & 166 & 272.0 \\
\hline 3 & 341.50 & 8111009 & 0.091 & 0.723 & 0.016 & 297 & 66 & -1.5 & 0.015 & 0.96 & 180 & 275.9 \\
\hline 4 & 336.88 & 10071720 & 0.208 & -9 & -9 & -999 & 231 & 38.7 & 0.075 & 2.56 & 170 & 301.4 \\
\hline 5 & 336.22 & 8110210 & 0.137 & 0.84 & 0.01 & 218 & 122 & -2.4 & 0.035 & 1.26 & 173 & 292.5 \\
\hline 6 & 326.31 & 9080319 & 0.286 & -9 & -9 & -999 & 369 & 94.6 & 0.075 & 3.46 & 172 & 300.9 \\
\hline 7 & 293.70 & 8121814 & 0.096 & 0.359 & 0.023 & 191 & 72 & -9.2 & 0.035 & 1.06 & 175 & 274.2 \\
\hline 8 & 290.01 & 10080319 & 0.217 & -9 & -9 & -999 & 245 & 60.4 & 0.075 & 2.64 & 173 & 307.0 \\
\hline 9 & 286.17 & 9102109 & 0.198 & 0.681 & 0.013 & 222 & 212 & -13.7 & 0.066 & 1.96 & 176 & 287.0 \\
\hline 10 & 285.28 & 8010404 & 0.18 & -9 & -9 & -999 & 184 & 24.8 & 0.035 & 2.62 & 172 & 268.8 \\
\hline 11 & 275.44 & 9102611 & 0.2 & 0.934 & 0.005 & 313 & 214 & -7.6 & 0.066 & 1.84 & 163 & 290.9 \\
\hline 12 & 272.86 & 9080514 & 0.124 & 0.732 & 0.006 & 328 & 106 & -4 & 0.075 & 1.01 & 165 & 298.8 \\
\hline 13 & 268.75 & 8060220 & 0.198 & -9 & -9 & -999 & 212 & 33.3 & 0.075 & 2.45 & 171 & 298.8 \\
\hline 14 & 267.48 & 8112324 & 0.219 & -9 & -9 & -999 & 246 & 75.6 & 0.035 & 3.1 & 172 & 278.8 \\
\hline 15 & 264.78 & 8122619 & 0.354 & -9 & -9 & -999 & 505 & 173.6 & 0.035 & 4.93 & 170 & 290.9 \\
\hline 16 & 261.50 & 8020501 & 0.4 & -9 & -9 & -999 & 607 & 132 & 0.035 & 5.6 & 172 & 291.4 \\
\hline 17 & 260.89 & 8120219 & 0.276 & -9 & -9 & -999 & 348 & 59.7 & 0.035 & 3.93 & 172 & 276.4 \\
\hline 18 & 258.62 & 9021719 & 0.382 & -9 & -9 & -999 & 567 & 230.9 & 0.035 & 5.29 & 170 & 279.2 \\
\hline 19 & 257.68 & 8061320 & 0.208 & -9 & -9 & -999 & 274 & 37.8 & 0.075 & 2.56 & 172 & 294.9 \\
\hline 20 & 254.62 & 8120822 & 0.415 & -9 & -9 & -999 & 640 & 137.5 & 0.035 & 5.8 & 170 & 282.5 \\
\hline 21 & 254.60 & 8010604 & 0.351 & -9 & -9 & -999 & 500 & 200.1 & 0.035 & 4.88 & 172 & 285.9 \\
\hline 22 & 252.02 & 8121321 & 0.431 & -9 & -9 & -999 & 678 & 146.8 & 0.035 & 6.02 & 172 & 279.2 \\
\hline 23 & 251.43 & 8020417 & 0.338 & -9 & -9 & -999 & 473 & 361.6 & 0.035 & 4.65 & 170 & 292.5 \\
\hline 24 & 246.75 & 10051220 & 0.198 & -9 & -9 & -999 & 212 & 32.9 & 0.04 & 2.78 & 172 & 297.0 \\
\hline 25 & 246.40 & 9042623 & 0.278 & -9 & -9 & -999 & 352 & 68 & 0.04 & 3.85 & 171 & 296.4 \\
\hline
\end{tabular}

Table 10 Meteorological Conditions for Top 25 Modeled Concentrations at Gibson East Mt. Carmel Monitor Using Beta $u^{\star}$ AERMET and Default AERMOD

\begin{tabular}{|c|c|c|c|c|c|c|c|c|c|c|c|c|}
\hline Rank & Conc. & YYMMDDHH & $u^{*}$ & $\mathbf{w}^{*}$ & VPTG & $Z_{i c}$ & $z_{\text {im }}$ & $\mathbf{L}$ & $z_{0}$ & WSPD & WDIR & temp \\
\hline 1 & 314.09 & 9061920 & 0.377 & -9 & -9 & -999 & 561 & 121.3 & 0.049 & 4.95 & 219 & 302.0 \\
\hline 2 & 282.35 & 10011012 & 0.156 & 0.809 & 0.018 & 209 & 147 & -3.7 & 0.035 & 1.53 & 222 & 265.4 \\
\hline 3 & 256.70 & 9061108 & 0.198 & 0.816 & 0.012 & 244 & 220 & -8.8 & 0.049 & 2.01 & 220 & 294.9 \\
\hline 4 & 250.44 & 9102310 & 0.604 & 0.653 & 0.006 & 249 & 1126 & -493.7 & 0.044 & 7.76 & 217 & 292.5 \\
\hline 5 & 246.85 & 10041310 & 0.219 & 0.994 & 0.005 & 300 & 246 & -8 & 0.04 & 2.3 & 216 & 297.5 \\
\hline 6 & 240.59 & 10082919 & 0.331 & -9 & -9 & -999 & 460 & 92.6 & 0.049 & 4.37 & 219 & 299.2 \\
\hline 7 & 236.25 & 10021111 & 0.147 & 1.118 & 0.014 & 302 & 136 & -1.7 & 0.035 & 1.28 & 231 & 269.9 \\
\hline 8 & 229.45 & 8052209 & 0.097 & 0.729 & 0.005 & 252 & 73 & -1.5 & 0.04 & 0.8 & 228 & 290.4 \\
\hline 9 & 228.12 & 8051718 & 0.377 & 0.673 & 0.005 & 2016 & 563 & -883.8 & 0.04 & 4.95 & 220 & 296.4 \\
\hline 10 & 221.70 & 10072101 & 0.327 & -9 & -9 & -999 & 448 & 103.6 & 0.049 & 4.31 & 218 & 299.9 \\
\hline 11 & 218.55 & 10081008 & 0.178 & 0.988 & 0.005 & 266 & 180 & -3.9 & 0.049 & 1.62 & 230 & 304.2 \\
\hline 12 & 218.10 & 10040111 & 0.548 & 1.052 & 0.006 & 326 & 974 & -115.3 & 0.04 & 6.96 & 215 & 297.0 \\
\hline 13 & 217.16 & 10060524 & 0.415 & -9 & -9 & -999 & 642 & 146 & 0.049 & 5.44 & 217 & 298.8 \\
\hline 14 & 214.18 & 10062624 & 0.287 & -9 & -9 & -999 & 369 & 69.7 & 0.049 & 3.81 & 219 & 299.9 \\
\hline 15 & 212.93 & 9090711 & 0.203 & 1.094 & 0.005 & 348 & 219 & -5.5 & 0.044 & 1.99 & 220 & 297.0 \\
\hline 16 & 211.49 & 10050518 & 0.429 & 1.219 & 0.009 & 2467 & 676 & -268 & 0.04 & 5.56 & 215 & 299.2 \\
\hline 17 & 207.38 & 10090218 & 0.275 & 0.362 & 0.005 & 2115 & 359 & -2338.8 & 0.044 & 3.57 & 222 & 301.4 \\
\hline 18 & 206.43 & 10041111 & 0.226 & 1.122 & 0.005 & 378 & 258 & -7.7 & 0.04 & 2.37 & 214 & 295.9 \\
\hline 19 & 205.22 & 10040721 & 0.456 & -9 & -9 & -999 & 740 & 341.3 & 0.04 & 6.12 & 217 & 289.2 \\
\hline 20 & 202.77 & 10072024 & 0.322 & -9 & -9 & -999 & 438 & 88 & 0.049 & 4.26 & 221 & 299.9 \\
\hline 21 & 201.72 & 9080918 & 0.407 & 1.058 & 0.005 & 1769 & 626 & -252 & 0.049 & 5.07 & 220 & 303.8 \\
\hline 22 & 201.21 & 10072301 & 0.244 & -9 & -9 & -999 & 290 & 50.5 & 0.049 & 3.27 & 219 & 298.8 \\
\hline 23 & 200.27 & 10051711 & 0.237 & 0.947 & 0.008 & 430 & 277 & -16.9 & 0.04 & 2.69 & 215 & 292.5 \\
\hline 24 & 198.08 & 9061619 & 0.41 & -9 & -9 & -999 & 638 & 303.5 & 0.049 & 5.29 & 218 & 295.9 \\
\hline 25 & 197.36 & 8120612 & 0.46 & 0.484 & 0.007 & 305 & 748 & -655.8 & 0.035 & 6.18 & 220 & 274.2 \\
\hline
\end{tabular}


Table 11 Meteorological Conditions for Top 25 Modeled Concentrations at Gibson Shrodt Monitor Using Beta $u^{*}$ AERMET and Default AERMOD

\begin{tabular}{|c|c|c|c|c|c|c|c|c|c|c|c|c|}
\hline Rank & Conc. & YYMMDDHH & $\mathrm{u}^{*}$ & $\mathbf{w}^{*}$ & VPTG & $Z_{i c}$ & $z_{\text {im }}$ & $\mathbf{L}$ & $z_{0}$ & WSPD & WDIR & temp \\
\hline 1 & 283.02 & 9102709 & 0.133 & 0.527 & 0.012 & 222 & 116 & -8.9 & 0.112 & 1.08 & 87 & 285.4 \\
\hline 2 & 280.21 & 10022110 & 0.119 & 0.583 & 0.012 & 231 & 98 & -4.9 & 0.026 & 1.3 & 87 & 281.4 \\
\hline 3 & 270.44 & 9042907 & 0.096 & 0.402 & 0.012 & 161 & 71 & -5.4 & 0.038 & 0.97 & 90 & 291.4 \\
\hline 4 & 244.03 & 9121610 & 0.161 & 0.624 & 0.029 & 177 & 156 & -7.7 & 0.026 & 1.86 & 92 & 268.8 \\
\hline 5 & 220.58 & 9121814 & 0.127 & 0.529 & 0.009 & 381 & 109 & -13.3 & 0.026 & 1.55 & 91 & 282.0 \\
\hline 6 & 207.35 & 8122511 & 0.222 & 0.777 & 0.028 & 332 & 251 & -19.4 & 0.026 & 2.79 & 88 & 271.4 \\
\hline 7 & 197.55 & 9050812 & 0.188 & 0.732 & 0.005 & 500 & 196 & -21.2 & 0.038 & 2.2 & 90 & 293.8 \\
\hline 8 & 184.82 & 10041210 & 0.187 & 0.937 & 0.009 & 255 & 194 & -5.1 & 0.038 & 1.88 & 96 & 294.9 \\
\hline 9 & 178.54 & 8021615 & 0.162 & 0.414 & 0.009 & 195 & 157 & -29.4 & 0.026 & 2.1 & 85 & 275.9 \\
\hline 10 & 172.89 & 9122311 & 0.239 & 0.843 & 0.013 & 273 & 281 & -15.6 & 0.026 & 2.95 & 97 & 282.0 \\
\hline 11 & 161.54 & 10012014 & 0.207 & 0.553 & 0.027 & 327 & 227 & -43.1 & 0.026 & 2.74 & 86 & 280.4 \\
\hline 12 & 158.31 & 9070907 & 0.178 & 0.565 & 0.009 & 215 & 180 & -16.7 & 0.124 & 1.52 & 91 & 294.9 \\
\hline 13 & 158.30 & 9080513 & 0.174 & 0.734 & 0.007 & 311 & 174 & -10.4 & 0.119 & 1.42 & 88 & 298.1 \\
\hline 14 & 155.64 & 8073010 & 0.243 & 0.943 & 0.005 & 272 & 288 & -11.6 & 0.124 & 1.99 & 96 & 301.4 \\
\hline 15 & 154.74 & 10021415 & 0.164 & 0.66 & 0.007 & 375 & 160 & -14.4 & 0.026 & 2.01 & 90 & 273.1 \\
\hline 16 & 143.48 & 10032514 & 0.265 & 0.56 & 0.011 & 316 & 327 & -83.7 & 0.038 & 3.36 & 86 & 285.4 \\
\hline 17 & 139.88 & 9021311 & 0.216 & 1.131 & 0.005 & 443 & 241 & -7.7 & 0.026 & 2.49 & 89 & 279.2 \\
\hline 18 & 139.58 & 8090407 & 0.23 & 0.486 & 0.015 & 186 & 264 & -49.2 & 0.117 & 2.2 & 93 & 295.9 \\
\hline 19 & 137.66 & 8092008 & 0.18 & 0.821 & 0.008 & 222 & 183 & -5.9 & 0.112 & 1.38 & 81 & 294.9 \\
\hline 20 & 136.81 & 10020413 & 0.279 & 1.331 & 0.015 & 472 & 354 & -10.8 & 0.026 & 3.32 & 88 & 276.4 \\
\hline 21 & 131.98 & 10020809 & 0.193 & 0.507 & 0.007 & 198 & 204 & -27.5 & 0.026 & 2.49 & 85 & 269.9 \\
\hline 22 & 129.32 & 9082807 & 0.097 & 0.418 & 0.013 & 164 & 72 & -5.1 & 0.124 & 0.7 & 100 & 295.4 \\
\hline 23 & 124.35 & 8013114 & 0.164 & 0.609 & 0.011 & 256 & 167 & -12.5 & 0.026 & 1.98 & 78 & 275.4 \\
\hline 24 & 123.73 & 10061009 & 0.234 & 1.084 & 0.005 & 349 & 272 & -8.8 & 0.124 & 1.84 & 93 & 300.9 \\
\hline 25 & 122.46 & 9092411 & 0.183 & 0.551 & 0.005 & 196 & 188 & -17.9 & 0.117 & 1.6 & 91 & 296.4 \\
\hline
\end{tabular}

Table 12 Meteorological Conditions for Top 25 Modeled Concentrations at Gibson Tower Monitor Using Beta $\mathbf{u}^{\star}$ AERMET and Default AERMOD

\begin{tabular}{|c|c|c|c|c|c|c|c|c|c|c|c|c|}
\hline Rank & Conc. & YYMMDDHH & $\mathrm{u}^{*}$ & $\mathbf{w}^{*}$ & VPTG & $z_{\text {ic }}$ & $z_{i m}$ & $\mathbf{L}$ & $\mathrm{z}_{0}$ & WSPD & WDIR & temp \\
\hline 1 & 440.35 & 9122113 & 0.069 & 0.544 & 0.005 & 337 & 44 & -1.8 & 0.018 & 0.72 & 295 & 274.9 \\
\hline 2 & 261.77 & 8112811 & 0.143 & 0.967 & 0.013 & 365 & 130 & -3 & 0.019 & 1.57 & 304 & 280.4 \\
\hline 3 & 243.51 & 9060207 & 0.135 & 0.766 & 0.016 & 199 & 120 & -2.8 & 0.047 & 1.18 & 304 & 297.5 \\
\hline 4 & 241.16 & 8081308 & 0.138 & 1.017 & 0.005 & 312 & 123 & -2 & 0.032 & 1.26 & 297 & 295.9 \\
\hline 5 & 237.06 & 9101210 & 0.11 & 0.834 & 0.01 & 242 & 87 & -1.4 & 0.027 & 0.99 & 293 & 288.1 \\
\hline 6 & 225.77 & 8070109 & 0.167 & 0.887 & 0.005 & 274 & 164 & -4.6 & 0.047 & 1.57 & 308 & 299.2 \\
\hline 7 & 216.48 & 10021911 & 0.139 & 1.216 & 0.01 & 351 & 124 & -1.3 & 0.018 & 1.37 & 278 & 277.0 \\
\hline 8 & 215.95 & 10050408 & 0.131 & 1.081 & 0.005 & 348 & 114 & -1.5 & 0.026 & 1.21 & 301 & 292.5 \\
\hline 9 & 185.94 & 10022612 & 0.413 & 1.712 & 0.011 & 801 & 637 & -28.1 & 0.018 & 5.7 & 298 & 275.9 \\
\hline 10 & 180.42 & 10091908 & 0.129 & 0.844 & 0.013 & 233 & 112 & -2.1 & 0.027 & 1.25 & 292 & 294.9 \\
\hline 11 & 179.89 & 9072309 & 0.205 & 1.006 & 0.005 & 422 & 223 & -8.9 & 0.047 & 2.1 & 308 & 297.0 \\
\hline 12 & 179.29 & 10081416 & 0.364 & 2.119 & 0.005 & 1790 & 527 & -22.7 & 0.047 & 4.09 & 303 & 309.9 \\
\hline 13 & 173.47 & 9070309 & 0.176 & 0.714 & 0.005 & 285 & 178 & -10.7 & 0.047 & 1.84 & 301 & 297.0 \\
\hline 14 & 171.69 & 10022512 & 0.258 & 1.085 & 0.011 & 302 & 314 & -10.1 & 0.019 & 3.25 & 309 & 272.5 \\
\hline 15 & 166.82 & 8100111 & 0.406 & 1.809 & 0.009 & 873 & 621 & -24.7 & 0.041 & 4.73 & 300 & 290.9 \\
\hline 16 & 166.45 & 10070909 & 0.221 & 0.811 & 0.005 & 299 & 249 & -15.1 & 0.047 & 2.39 & 306 & 298.8 \\
\hline 17 & 159.40 & 9062014 & 0.309 & 1.889 & 0.005 & 1573 & 412 & -17.1 & 0.032 & 3.68 & 298 & 305.4 \\
\hline 18 & 156.25 & 9061717 & 0.29 & 1.936 & 0.005 & 1762 & 376 & -14.8 & 0.032 & 3.41 & 298 & 305.9 \\
\hline 19 & 156.10 & 10041912 & 0.184 & 1.328 & 0.005 & 571 & 189 & -3.8 & 0.025 & 1.96 & 296 & 290.9 \\
\hline 20 & 153.22 & 10072909 & 0.241 & 1.108 & 0.005 & 393 & 283 & -10.1 & 0.047 & 2.5 & 310 & 302.5 \\
\hline 21 & 152.38 & 8061908 & 0.118 & 0.838 & 0.008 & 232 & 97 & -1.6 & 0.047 & 0.94 & 306 & 296.4 \\
\hline 22 & 146.04 & 8072110 & 0.224 & 1.187 & 0.005 & 536 & 254 & -9 & 0.032 & 2.5 & 293 & 305.9 \\
\hline 23 & 143.80 & 10062113 & 0.257 & 1.802 & 0.005 & 1146 & 317 & -8.3 & 0.032 & 2.86 & 298 & 307.0 \\
\hline 24 & 143.78 & 9073117 & 0.297 & 1.362 & 0.005 & 1627 & 389 & -42.3 & 0.047 & 3.49 & 302 & 299.2 \\
\hline 25 & 143.07 & 9050410 & 0.121 & 0.959 & 0.005 & 354 & 100 & -1.8 & 0.026 & 1.14 & 321 & 289.9 \\
\hline
\end{tabular}


Table 13 Meteorological Conditions for Top 25 Modeled Concentrations at Gibson Mt. Carmel Monitor Using Beta $u^{\star}$ AERMET and Beta AERMOD with Minimum Sigma-v $=0.5 \mathrm{~m} / \mathrm{s}$

\begin{tabular}{|c|c|c|c|c|c|c|c|c|c|c|c|c|}
\hline Rank & Conc. & YYMMDDHH & $\mathrm{u}^{*}$ & $\mathrm{w}^{*}$ & VPTG & $Z_{i c}$ & $z_{\text {im }}$ & $\mathbf{L}$ & $z_{0}$ & WSPD & WDIR & temp \\
\hline 1 & 514.52 & 10062210 & 0.293 & 1.296 & 0.005 & 487 & 381 & -14.1 & 0.075 & 2.81 & 155 & 303.8 \\
\hline 2 & 391.86 & 9121612 & 0.156 & 0.858 & 0.024 & 261 & 148 & -4 & 0.035 & 1.55 & 166 & 272.0 \\
\hline 3 & 328.26 & 8110210 & 0.137 & 0.84 & 0.01 & 218 & 122 & -2.4 & 0.035 & 1.26 & 173 & 292.5 \\
\hline 4 & 326.31 & 9080319 & 0.286 & -9 & -9 & -999 & 369 & 94.6 & 0.075 & 3.46 & 172 & 300.9 \\
\hline 5 & 316.23 & 10071722 & 0.232 & -9 & -9 & -999 & 268 & 45.8 & 0.075 & 2.84 & 173 & 300.9 \\
\hline 6 & 291.91 & 8111009 & 0.091 & 0.723 & 0.016 & 297 & 66 & -1.5 & 0.015 & 0.96 & 180 & 275.9 \\
\hline 7 & 282.51 & 9102109 & 0.198 & 0.681 & 0.013 & 222 & 212 & -13.7 & 0.066 & 1.96 & 176 & 287.0 \\
\hline 8 & 271.45 & 9102611 & 0.2 & 0.934 & 0.005 & 313 & 214 & -7.6 & 0.066 & 1.84 & 163 & 290.9 \\
\hline 9 & 265.19 & 10080319 & 0.217 & -9 & -9 & -999 & 245 & 60.4 & 0.075 & 2.64 & 173 & 307.0 \\
\hline 10 & 264.78 & 8122619 & 0.354 & -9 & -9 & -999 & 505 & 173.6 & 0.035 & 4.93 & 170 & 290.9 \\
\hline 11 & 261.50 & 8020501 & 0.4 & -9 & -9 & -999 & 607 & 132 & 0.035 & 5.6 & 172 & 291.4 \\
\hline 12 & 260.89 & 8120219 & 0.276 & -9 & -9 & -999 & 348 & 59.7 & 0.035 & 3.93 & 172 & 276.4 \\
\hline 13 & 259.54 & 9080514 & 0.124 & 0.732 & 0.006 & 328 & 106 & -4 & 0.075 & 1.01 & 165 & 298.8 \\
\hline 14 & 258.62 & 9021719 & 0.382 & -9 & -9 & -999 & 567 & 230.9 & 0.035 & 5.29 & 170 & 279.2 \\
\hline 15 & 254.62 & 8120822 & 0.415 & -9 & -9 & -999 & 640 & 137.5 & 0.035 & 5.8 & 170 & 282.5 \\
\hline 16 & 254.60 & 8010604 & 0.351 & -9 & -9 & -999 & 500 & 200.1 & 0.035 & 4.88 & 172 & 285.9 \\
\hline 17 & 252.06 & 8010404 & 0.18 & -9 & -9 & -999 & 184 & 24.8 & 0.035 & 2.62 & 172 & 268.8 \\
\hline 18 & 252.02 & 8121321 & 0.431 & -9 & -9 & -999 & 678 & 146.8 & 0.035 & 6.02 & 172 & 279.2 \\
\hline 19 & 251.43 & 8020417 & 0.338 & -9 & -9 & -999 & 473 & 361.6 & 0.035 & 4.65 & 170 & 292.5 \\
\hline 20 & 246.40 & 9042623 & 0.278 & -9 & -9 & -999 & 352 & 68 & 0.04 & 3.85 & 171 & 296.4 \\
\hline 21 & 245.76 & 10033121 & 0.316 & -9 & -9 & -999 & 427 & 83.2 & 0.04 & 4.36 & 171 & 293.8 \\
\hline 22 & 244.53 & 8112324 & 0.219 & -9 & -9 & -999 & 246 & 75.6 & 0.035 & 3.1 & 172 & 278.8 \\
\hline 23 & 238.84 & 8060220 & 0.198 & -9 & -9 & -999 & 212 & 33.3 & 0.075 & 2.45 & 171 & 298.8 \\
\hline 24 & 236.32 & 8120905 & 0.414 & -9 & -9 & -999 & 640 & 274.4 & 0.035 & 5.72 & 173 & 282.0 \\
\hline 25 & 235.09 & 8061320 & 0.208 & -9 & -9 & -999 & 274 & 37.8 & 0.075 & 2.56 & 172 & 294.9 \\
\hline
\end{tabular}

Table 14 Meteorological Conditions for Top 25 Modeled Concentrations at Gibson East Mt. Carmel Monitor Using Beta $u^{\star}$ AERMET and Beta AERMOD with Minimum Sigma-v $=0.5 \mathrm{~m} / \mathrm{s}$

\begin{tabular}{|c|c|c|c|c|c|c|c|c|c|c|c|c|}
\hline Rank & Conc. & YYMMDDHH & $u^{*}$ & $\mathrm{w}^{*}$ & VPTG & $z_{i c}$ & $z_{i m}$ & L & $z_{0}$ & WSPD & WDIR & temp \\
\hline 1 & 314.09 & 9061920 & 0.377 & -9 & -9 & -999 & 561 & 121.3 & 0.049 & 4.95 & 219 & 302.0 \\
\hline 2 & 277.06 & 10011012 & 0.156 & 0.809 & 0.018 & 209 & 147 & -3.7 & 0.035 & 1.53 & 222 & 265.4 \\
\hline 3 & 253.20 & 9061108 & 0.198 & 0.816 & 0.012 & 244 & 220 & -8.8 & 0.049 & 2.01 & 220 & 294.9 \\
\hline 4 & 250.31 & 9102310 & 0.604 & 0.653 & 0.006 & 249 & 1126 & -493.7 & 0.044 & 7.76 & 217 & 292.5 \\
\hline 5 & 243.88 & 10041310 & 0.219 & 0.994 & 0.005 & 300 & 246 & -8 & 0.04 & 2.3 & 216 & 297.5 \\
\hline 6 & 240.59 & 10082919 & 0.331 & -9 & -9 & -999 & 460 & 92.6 & 0.049 & 4.37 & 219 & 299.2 \\
\hline 7 & 230.21 & 10021111 & 0.147 & 1.118 & 0.014 & 302 & 136 & -1.7 & 0.035 & 1.28 & 231 & 269.9 \\
\hline 8 & 228.02 & 8051718 & 0.377 & 0.673 & 0.005 & 2016 & 563 & -883.8 & 0.04 & 4.95 & 220 & 296.4 \\
\hline 9 & 221.70 & 10072101 & 0.327 & -9 & -9 & -999 & 448 & 103.6 & 0.049 & 4.31 & 218 & 299.9 \\
\hline 10 & 218.03 & 10040111 & 0.548 & 1.052 & 0.006 & 326 & 974 & -115.3 & 0.04 & 6.96 & 215 & 297.0 \\
\hline 11 & 217.16 & 10060524 & 0.415 & -9 & -9 & -999 & 642 & 146 & 0.049 & 5.44 & 217 & 298.8 \\
\hline 12 & 214.84 & 10081008 & 0.178 & 0.988 & 0.005 & 266 & 180 & -3.9 & 0.049 & 1.62 & 230 & 304.2 \\
\hline 13 & 214.18 & 10062624 & 0.287 & -9 & -9 & -999 & 369 & 69.7 & 0.049 & 3.81 & 219 & 299.9 \\
\hline 14 & 211.26 & 10050518 & 0.429 & 1.219 & 0.009 & 2467 & 676 & -268 & 0.04 & 5.56 & 215 & 299.2 \\
\hline 15 & 209.80 & 9090711 & 0.203 & 1.094 & 0.005 & 348 & 219 & -5.5 & 0.044 & 1.99 & 220 & 297.0 \\
\hline 16 & 207.31 & 10090218 & 0.275 & 0.362 & 0.005 & 2115 & 359 & -2338.8 & 0.044 & 3.57 & 222 & 301.4 \\
\hline 17 & 205.22 & 10040721 & 0.456 & -9 & -9 & $\begin{array}{l}-999 \\
\end{array}$ & 740 & 341.3 & 0.04 & 6.12 & 217 & 289.2 \\
\hline 18 & 203.95 & 10041111 & 0.226 & 1.122 & 0.005 & 378 & 258 & -7.7 & 0.04 & 2.37 & 214 & 295.9 \\
\hline 19 & 202.77 & 10072024 & 0.322 & -9 & -9 & -999 & 438 & 88 & 0.049 & 4.26 & 221 & 299.9 \\
\hline 20 & 201.59 & 9080918 & 0.407 & 1.058 & 0.005 & 1769 & 626 & -252 & 0.049 & 5.07 & 220 & 303.8 \\
\hline 21 & 199.84 & 10072301 & 0.244 & -9 & -9 & -999 & 290 & 50.5 & 0.049 & 3.27 & 219 & 298.8 \\
\hline 22 & 198.25 & 10051711 & 0.237 & 0.947 & 0.008 & 430 & 277 & -16.9 & 0.04 & 2.69 & 215 & 292.5 \\
\hline 23 & 198.08 & 9061619 & 0.41 & -9 & -9 & -999 & 638 & 303.5 & 0.049 & 5.29 & 218 & 295.9 \\
\hline 24 & 197.31 & 8120612 & 0.46 & 0.484 & 0.007 & 305 & 748 & -655.8 & 0.035 & 6.18 & 220 & 274.2 \\
\hline 25 & 197.13 & 10062320 & 0.35 & -9 & -9 & -999 & 506 & 104.3 & 0.049 & 4.61 & 221 & 301.4 \\
\hline
\end{tabular}


Table 15 Meteorological Conditions for Top 25 Modeled Concentrations at Gibson Shrodt Monitor Using Beta $\mathrm{u}^{\star}$ AERMET and Beta AERMOD with Minimum Sigma-v $=0.5 \mathrm{~m} / \mathrm{s}$

\begin{tabular}{|c|c|c|c|c|c|c|c|c|c|c|c|c|}
\hline Rank & Conc. & YYMMDDHH & $u^{*}$ & $\mathbf{w}^{*}$ & VPTG & $\mathrm{Z}_{\mathrm{ic}}$ & $z_{\text {im }}$ & $\mathbf{L}$ & $\mathrm{z}_{0}$ & WSPD & WDIR & temp \\
\hline 1 & 228.26 & 9121610 & 0.161 & 0.624 & 0.029 & 177 & 156 & -7.7 & 0.026 & 1.86 & 92 & 268.8 \\
\hline 2 & 214.39 & 10022110 & 0.119 & 0.583 & 0.012 & 231 & 98 & -4.9 & 0.026 & 1.3 & 87 & 281.4 \\
\hline 3 & 205.54 & 9102709 & 0.133 & 0.527 & 0.012 & 222 & 116 & -8.9 & 0.112 & 1.08 & 87 & 285.4 \\
\hline 4 & 203.41 & 8122511 & 0.222 & 0.777 & 0.028 & 332 & 251 & -19.4 & 0.026 & 2.79 & 88 & 271.4 \\
\hline 5 & 192.92 & 9050812 & 0.188 & 0.732 & 0.005 & 500 & 196 & -21.2 & 0.038 & 2.2 & 90 & 293.8 \\
\hline 6 & 189.23 & 9121811 & 0.117 & 0.745 & 0.013 & 193 & 95 & -1.8 & 0.026 & 1.11 & 93 & 278.8 \\
\hline 7 & 179.49 & 10041210 & 0.187 & 0.937 & 0.009 & 255 & 194 & -5.1 & 0.038 & 1.88 & 96 & 294.9 \\
\hline 8 & 169.90 & 9122311 & 0.239 & 0.843 & 0.013 & 273 & 281 & -15.6 & 0.026 & 2.95 & 97 & 282.0 \\
\hline 9 & 158.53 & 10012014 & 0.207 & 0.553 & 0.027 & 327 & 227 & -43.1 & 0.026 & 2.74 & 86 & 280.4 \\
\hline 10 & 152.53 & 9080513 & 0.174 & 0.734 & 0.007 & 311 & 174 & -10.4 & 0.119 & 1.42 & 88 & 298.1 \\
\hline 11 & 151.79 & 8073010 & 0.243 & 0.943 & 0.005 & 272 & 288 & -11.6 & 0.124 & 1.99 & 96 & 301.4 \\
\hline 12 & 150.48 & 10021415 & 0.164 & 0.66 & 0.007 & 375 & 160 & -14.4 & 0.026 & 2.01 & 90 & 273.1 \\
\hline 13 & 145.44 & 9070907 & 0.178 & 0.565 & 0.009 & 215 & 180 & -16.7 & 0.124 & 1.52 & 91 & 294.9 \\
\hline 14 & 141.41 & 10032514 & 0.265 & 0.56 & 0.011 & 316 & 327 & -83.7 & 0.038 & 3.36 & 86 & 285.4 \\
\hline 15 & 140.91 & 8021615 & 0.162 & 0.414 & 0.009 & 195 & 157 & -29.4 & 0.026 & 2.1 & 85 & 275.9 \\
\hline 16 & 136.75 & 9021311 & 0.216 & 1.131 & 0.005 & 443 & 241 & -7.7 & 0.026 & 2.49 & 89 & 279.2 \\
\hline 17 & 136.71 & 8090407 & 0.23 & 0.486 & 0.015 & 186 & 264 & -49.2 & 0.117 & 2.2 & 93 & 295.9 \\
\hline 18 & 134.53 & 10020413 & 0.279 & 1.331 & 0.015 & 472 & 354 & -10.8 & 0.026 & 3.32 & 88 & 276.4 \\
\hline 19 & 133.96 & 9042907 & 0.096 & 0.402 & 0.012 & 161 & 71 & -5.4 & 0.038 & 0.97 & 90 & 291.4 \\
\hline 20 & 132.41 & 8092008 & 0.18 & 0.821 & 0.008 & 222 & 183 & -5.9 & 0.112 & 1.38 & 81 & 294.9 \\
\hline 21 & 123.43 & 10020809 & 0.193 & 0.507 & 0.007 & 198 & 204 & -27.5 & 0.026 & 2.49 & 85 & 269.9 \\
\hline 22 & 120.72 & 8013114 & 0.164 & 0.609 & 0.011 & 256 & 167 & -12.5 & 0.026 & 1.98 & 78 & 275.4 \\
\hline 23 & 120.16 & 10061009 & 0.234 & 1.084 & 0.005 & 349 & 272 & -8.8 & 0.124 & 1.84 & 93 & 300.9 \\
\hline 24 & 118.90 & 10091009 & 0.274 & 0.717 & 0.012 & 300 & 344 & -41.8 & 0.112 & 2.62 & 82 & 290.9 \\
\hline 25 & 113.10 & 9092411 & 0.183 & 0.551 & 0.005 & 196 & 188 & -17.9 & 0.117 & 1.6 & 91 & 296.4 \\
\hline
\end{tabular}

Table 16 Meteorological Conditions for Top 25 Modeled Concentrations at Gibson Tower Monitor Using Beta $u^{\star}$ AERMET and Beta AERMOD with Minimum Sigma-v $=0.5 \mathrm{~m} / \mathrm{s}$

\begin{tabular}{|c|c|c|c|c|c|c|c|c|c|c|c|c|}
\hline Rank & Conc. & YYMMDDHH & $\mathrm{u}^{*}$ & $\mathbf{w}^{*}$ & VPTG & $Z_{i c}$ & $z_{i m}$ & $\mathbf{L}$ & $\mathrm{z}_{0}$ & WSPD & WDIR & temp \\
\hline 1 & 257.81 & 8112811 & 0.143 & 0.967 & 0.013 & 365 & 130 & -3 & 0.019 & 1.57 & 304 & 280.4 \\
\hline 2 & 238.79 & 9060207 & 0.135 & 0.766 & 0.016 & 199 & 120 & -2.8 & 0.047 & 1.18 & 304 & 297.5 \\
\hline 3 & 236.56 & 8081308 & 0.138 & 1.017 & 0.005 & 312 & 123 & -2 & 0.032 & 1.26 & 297 & 295.9 \\
\hline 4 & 230.98 & 9101210 & 0.11 & 0.834 & 0.01 & 242 & 87 & -1.4 & 0.027 & 0.99 & 293 & 288.1 \\
\hline 5 & 222.77 & 8070109 & 0.167 & 0.887 & 0.005 & 274 & 164 & -4.6 & 0.047 & 1.57 & 308 & 299.2 \\
\hline 6 & 212.85 & 10021911 & 0.139 & 1.216 & 0.01 & 351 & 124 & -1.3 & 0.018 & 1.37 & 278 & 277.0 \\
\hline 7 & 211.34 & 10050408 & 0.131 & 1.081 & 0.005 & 348 & 114 & -1.5 & 0.026 & 1.21 & 301 & 292.5 \\
\hline 8 & 197.62 & 9122113 & 0.069 & 0.544 & 0.005 & 337 & 44 & -1.8 & 0.018 & 0.72 & 295 & 274.9 \\
\hline 9 & 185.29 & 10022612 & 0.413 & 1.712 & 0.011 & 801 & 637 & -28.1 & 0.018 & 5.7 & 298 & 275.9 \\
\hline 10 & 178.43 & 10081416 & 0.364 & 2.119 & 0.005 & 1790 & 527 & -22.7 & 0.047 & 4.09 & 303 & 309.9 \\
\hline 11 & 178.10 & 9072309 & 0.205 & 1.006 & 0.005 & 422 & 223 & -8.9 & 0.047 & 2.1 & 308 & 297.0 \\
\hline 12 & 177.15 & 10091908 & 0.129 & 0.844 & 0.013 & 233 & 112 & -2.1 & 0.027 & 1.25 & 292 & 294.9 \\
\hline 13 & 171.54 & 9070309 & 0.176 & 0.714 & 0.005 & 285 & 178 & -10.7 & 0.047 & 1.84 & 301 & 297.0 \\
\hline 14 & 170.62 & 10022512 & 0.258 & 1.085 & 0.011 & 302 & 314 & -10.1 & 0.019 & 3.25 & 309 & 272.5 \\
\hline 15 & 166.13 & 8100111 & 0.406 & 1.809 & 0.009 & 873 & 621 & -24.7 & 0.041 & 4.73 & 300 & 290.9 \\
\hline 16 & 165.10 & 10070909 & 0.221 & 0.811 & 0.005 & 299 & 249 & -15.1 & 0.047 & 2.39 & 306 & 298.8 \\
\hline 17 & 158.51 & 9062014 & 0.309 & 1.889 & 0.005 & 1573 & 412 & -17.1 & 0.032 & 3.68 & 298 & 305.4 \\
\hline 18 & 155.29 & 9061717 & 0.29 & 1.936 & 0.005 & 1762 & 376 & -14.8 & 0.032 & 3.41 & 298 & 305.9 \\
\hline 19 & 154.21 & 10041912 & 0.184 & 1.328 & 0.005 & 571 & 189 & -3.8 & 0.025 & 1.96 & 296 & 290.9 \\
\hline 20 & 151.99 & 10072909 & 0.241 & 1.108 & 0.005 & 393 & 283 & -10.1 & 0.047 & 2.5 & 310 & 302.5 \\
\hline 21 & 148.32 & 8061908 & 0.118 & 0.838 & 0.008 & 232 & 97 & -1.6 & 0.047 & 0.94 & 306 & 296.4 \\
\hline 22 & 144.82 & 8072110 & 0.224 & 1.187 & 0.005 & 536 & 254 & -9 & 0.032 & 2.5 & 293 & 305.9 \\
\hline 23 & 143.00 & 9073117 & 0.297 & 1.362 & 0.005 & 1627 & 389 & -42.3 & 0.047 & 3.49 & 302 & 299.2 \\
\hline 24 & 142.72 & 10062113 & 0.257 & 1.802 & 0.005 & 1146 & 317 & -8.3 & 0.032 & 2.86 & 298 & 307.0 \\
\hline 25 & 140.67 & 10091116 & 0.49 & 1.706 & 0.011 & 1148 & 822 & -67.8 & 0.041 & 6.06 & 301 & 300.9 \\
\hline
\end{tabular}


Table 17 Meteorological Conditions for Top 25 Modeled Concentrations at Gibson Mt. Carmel Monitor Using Beta $u^{\star}$ AERMET and SHARP

\begin{tabular}{|c|c|c|c|c|c|c|c|c|c|c|c|c|}
\hline Rank & Conc. & YYMMDDHH & $\mathrm{u}^{*}$ & $\mathbf{w}^{*}$ & VPTG & $z_{\text {ic }}$ & $z_{i m}$ & $\mathbf{L}$ & $\mathrm{z}_{0}$ & WSPD & WDIR & temp \\
\hline 1 & 505.99 & 10062209 & 0.319 & 1.063 & 0.005 & 330 & 432 & -22.2 & 0.11 & 2.9 & 147 & 302.5 \\
\hline 2 & 245.76 & 10033121 & 0.316 & -9 & -9 & -999 & 427 & 83.2 & 0.04 & 4.36 & 171 & 293.8 \\
\hline 3 & 239.28 & 8122619 & 0.354 & -9 & -9 & -999 & 505 & 173.6 & 0.035 & 4.93 & 170 & 290.9 \\
\hline 4 & 239.25 & 8020501 & 0.4 & -9 & -9 & -999 & 607 & 132 & 0.035 & 5.6 & 172 & 291.4 \\
\hline 5 & 237.20 & 9121612 & 0.156 & 0.858 & 0.024 & 261 & 148 & -4 & 0.035 & 1.55 & 166 & 272.0 \\
\hline 6 & 236.02 & 8120909 & 0.44 & 0.091 & 0.008 & 34 & 700 & -8888 & 0.035 & 5.96 & 172 & 281.4 \\
\hline 7 & 231.23 & 8010604 & 0.351 & -9 & -9 & -999 & 500 & 200.1 & 0.035 & 4.88 & 172 & 285.9 \\
\hline 8 & 227.45 & 10071722 & 0.232 & -9 & -9 & -999 & 268 & 45.8 & 0.075 & 2.84 & 173 & 300.9 \\
\hline 9 & 222.94 & 8060420 & 0.451 & -9 & -9 & -999 & 730 & 197.9 & 0.075 & 5.36 & 169 & 300.9 \\
\hline 10 & 209.86 & 8120219 & 0.276 & -9 & -9 & -999 & 348 & 59.7 & 0.035 & 3.93 & 172 & 276.4 \\
\hline 11 & 207.37 & 8012524 & 0.238 & -9 & -9 & -999 & 278 & 87 & 0.035 & 3.36 & 170 & 271.4 \\
\hline 12 & 203.57 & 8110210 & 0.137 & 0.84 & 0.01 & 218 & 122 & -2.4 & 0.035 & 1.26 & 173 & 292.5 \\
\hline 13 & 203.44 & 9042701 & 0.268 & -9 & -9 & -999 & 332 & 62.6 & 0.04 & 3.71 & 173 & 294.9 \\
\hline 14 & 202.41 & 8121321 & 0.431 & -9 & -9 & -999 & 678 & 146.8 & 0.035 & 6.02 & 172 & 279.2 \\
\hline 15 & 199.49 & 9080319 & 0.286 & -9 & -9 & -999 & 369 & 94.6 & 0.075 & 3.46 & 172 & 300.9 \\
\hline 16 & 198.35 & 10032411 & 0.252 & 0.822 & 0.005 & 236 & 304 & -17 & 0.04 & 2.86 & 165 & 291.4 \\
\hline 17 & 197.36 & 10012709 & 0.098 & 0.473 & 0.021 & 214 & 73 & -4.7 & 0.015 & 1.2 & 188 & 271.4 \\
\hline 18 & 196.01 & 10032721 & 0.32 & -9 & -9 & -999 & 434 & 140.5 & 0.04 & 4.36 & 170 & 288.8 \\
\hline 19 & 195.15 & 8020424 & 0.376 & -9 & -9 & -999 & 553 & 133.5 & 0.035 & 5.26 & 171 & 292.0 \\
\hline 20 & 192.87 & 8010721 & 0.402 & -9 & -9 & -999 & 612 & 133 & 0.035 & 5.63 & 171 & 290.4 \\
\hline 21 & 191.56 & 9042623 & 0.278 & -9 & -9 & -999 & 352 & 68 & 0.04 & 3.85 & 171 & 296.4 \\
\hline 22 & 191.34 & 8010417 & 0.337 & -9 & -9 & -999 & 470 & 90.2 & 0.035 & 4.75 & 174 & 277.5 \\
\hline 23 & 190.88 & 10090619 & 0.265 & -9 & -9 & -999 & 331 & 59.9 & 0.066 & 3.32 & 173 & 301.4 \\
\hline 24 & 190.10 & 8010807 & 0.52 & -9 & -9 & -999 & 901 & 445.6 & 0.035 & 7.13 & 172 & 290.4 \\
\hline 25 & 189.30 & 10040120 & 0.204 & -9 & -9 & -999 & 221 & 34.7 & 0.04 & 2.86 & 170 & 294.9 \\
\hline
\end{tabular}

Table 18 Meteorological Conditions for Top 25 Modeled Concentrations at Gibson East Mt. Carmel Monitor Using Beta $u^{\star}$ AERMET and SHARP

\begin{tabular}{|c|c|c|c|c|c|c|c|c|c|c|c|c|}
\hline Rank & Conc. & YYMMDDHH & $u^{*}$ & $\mathbf{w}^{*}$ & VPTG & $Z_{\text {ic }}$ & $z_{i m}$ & $\mathbf{L}$ & $z_{0}$ & WSPD & WDIR & temp \\
\hline 1 & 285.06 & 10021112 & 0.172 & 1.312 & 0.012 & 424 & 172 & -2.4 & 0.035 & 1.59 & 222 & 270.9 \\
\hline 2 & 235.67 & 10011012 & 0.156 & 0.809 & 0.018 & 209 & 147 & -3.7 & 0.035 & 1.53 & 222 & 265.4 \\
\hline 3 & 222.61 & 9061920 & 0.364 & -9 & -9 & -999 & 535 & 113.4 & 0.049 & 4.95 & 219 & 302.0 \\
\hline 4 & 219.33 & 10050518 & 0.429 & 1.219 & 0.009 & 2467 & 676 & -268 & 0.04 & 5.56 & 215 & 299.2 \\
\hline 5 & 218.10 & 10040111 & 0.548 & 1.052 & 0.006 & 326 & 974 & -115.3 & 0.04 & 6.96 & 215 & 297.0 \\
\hline 6 & 215.85 & 10080909 & 0.189 & 1.495 & 0.005 & 599 & 197 & -3 & 0.049 & 1.65 & 213 & 305.4 \\
\hline 7 & 204.91 & 10082919 & 0.314 & -9 & -9 & -999 & 429 & 83.7 & 0.049 & 4.37 & 219 & 299.2 \\
\hline 8 & 191.49 & 8080107 & 0.102 & 0.813 & 0.015 & 353 & 79 & -1.8 & 0.049 & 0.82 & 220 & 298.1 \\
\hline 9 & 188.19 & 10092309 & 0.417 & 1.053 & 0.005 & 243 & 647 & -37.8 & 0.044 & 4.93 & 220 & 302.5 \\
\hline 10 & 186.20 & 10060523 & 0.384 & -9 & -9 & -999 & 571 & 124.8 & 0.049 & 5.19 & 217 & 298.8 \\
\hline 11 & 183.63 & 10072218 & 0.36 & 1.167 & 0.005 & 1596 & 521 & -117 & 0.049 & 4.39 & 217 & 305.4 \\
\hline 12 & 182.65 & 10062624 & 0.265 & -9 & -9 & -999 & 328 & 59.6 & 0.049 & 3.81 & 219 & 299.9 \\
\hline 13 & 181.58 & 10051318 & 0.432 & 1.233 & 0.005 & 1643 & 690 & -176.8 & 0.04 & 5.56 & 217 & 299.2 \\
\hline 14 & 180.50 & 8111009 & 0.091 & 0.725 & 0.016 & 300 & 66 & -1.5 & 0.015 & 0.96 & 180 & 275.9 \\
\hline 15 & 180.45 & 9102310 & 0.604 & 0.653 & 0.006 & 249 & 1126 & -493.7 & 0.044 & 7.76 & 217 & 292.5 \\
\hline 16 & 179.71 & 9080919 & 0.312 & -9 & -9 & -999 & 425 & 98 & 0.049 & 4.29 & 217 & 301.4 \\
\hline 17 & 179.20 & 10091517 & 0.432 & 1.553 & 0.006 & 1877 & 681 & -101 & 0.044 & 5.36 & 218 & 304.2 \\
\hline 18 & 177.44 & 10072011 & 0.518 & 1.19 & 0.006 & 689 & 894 & -141.9 & 0.049 & 6.36 & 215 & 300.9 \\
\hline 19 & 177.03 & 10080311 & 0.384 & 2.068 & 0.005 & 1079 & 572 & -17.3 & 0.049 & 4.17 & 214 & 305.9 \\
\hline 20 & 173.21 & 10071803 & 0.271 & -9 & -9 & -999 & 338 & 62 & 0.049 & 3.88 & 217 & 298.1 \\
\hline 21 & 171.85 & 9062523 & 0.254 & -9 & -9 & -999 & 307 & 55 & 0.049 & 3.69 & 217 & 300.9 \\
\hline 22 & 170.70 & 10012710 & 0.209 & 0.797 & 0.022 & 301 & 229 & -13.5 & 0.015 & 2.83 & 205 & 273.1 \\
\hline 23 & 169.96 & 10010615 & 0.141 & 0.706 & 0.012 & 298 & 127 & -6 & 0.035 & 1.48 & 235 & 271.4 \\
\hline 24 & 169.80 & 9090711 & 0.203 & 1.094 & 0.005 & 348 & 219 & -5.5 & 0.044 & 1.99 & 220 & 297.0 \\
\hline 25 & 169.53 & 10051711 & 0.237 & 0.947 & 0.008 & 430 & 277 & -16.9 & 0.04 & 2.69 & 215 & 292.5 \\
\hline
\end{tabular}


Table 19 Meteorological Conditions for Top 25 Modeled Concentrations at Gibson Shrodt Monitor Using Beta $u^{*}$ AERMET and SHARP

\begin{tabular}{|c|c|c|c|c|c|c|c|c|c|c|c|c|}
\hline Rank & Conc. & YYMMDDHH & $\mathrm{u}^{*}$ & $\mathbf{w}^{*}$ & VPTG & $z_{\text {ic }}$ & $z_{i m}$ & $\mathbf{L}$ & $\mathrm{z}_{0}$ & WSPD & WDIR & temp \\
\hline 1 & 204.46 & 10021416 & 0.136 & 0.495 & 0.008 & 398 & 121 & -20.7 & 0.026 & 1.72 & 92 & 273.1 \\
\hline 2 & 168.19 & 9102709 & 0.133 & 0.527 & 0.012 & 222 & 116 & -8.9 & 0.112 & 1.08 & 87 & 285.4 \\
\hline 3 & 161.40 & 9122311 & 0.239 & 0.843 & 0.013 & 273 & 281 & -15.6 & 0.026 & 2.95 & 97 & 282.0 \\
\hline 4 & 153.05 & 10012014 & 0.207 & 0.553 & 0.027 & 327 & 227 & -43.1 & 0.026 & 2.74 & 86 & 280.4 \\
\hline 5 & 151.53 & 10032514 & 0.265 & 0.56 & 0.011 & 316 & 327 & -83.7 & 0.038 & 3.36 & 86 & 285.4 \\
\hline 6 & 139.25 & 10020413 & 0.279 & 1.331 & 0.015 & 472 & 354 & -10.8 & 0.026 & 3.32 & 88 & 276.4 \\
\hline 7 & 124.16 & 8090808 & 0.258 & 0.892 & 0.006 & 242 & 315 & -14.7 & 0.117 & 2.21 & 113 & 293.8 \\
\hline 8 & 119.06 & 10051210 & 0.168 & 0.995 & 0.021 & 563 & 166 & -6.8 & 0.038 & 1.76 & 101 & 294.2 \\
\hline 9 & 118.00 & 8090407 & 0.23 & 0.486 & 0.015 & 186 & 264 & -49.2 & 0.117 & 2.2 & 93 & 295.9 \\
\hline 10 & 117.62 & 8021615 & 0.162 & 0.414 & 0.009 & 195 & 157 & -29.4 & 0.026 & 2.1 & 85 & 275.9 \\
\hline 11 & 117.14 & 8122511 & 0.222 & 0.777 & 0.028 & 332 & 251 & -19.4 & 0.026 & 2.79 & 88 & 271.4 \\
\hline 12 & 112.83 & 10052911 & 0.315 & 2.53 & 0.008 & 1930 & 423 & -9.3 & 0.038 & 3.4 & 91 & 302.5 \\
\hline 13 & 112.07 & 8020313 & 0.279 & 0.885 & 0.012 & 303 & 354 & -23.8 & 0.026 & 3.56 & 93 & 284.2 \\
\hline 14 & 110.43 & 8020514 & 0.285 & 0.945 & 0.028 & 395 & 365 & -27 & 0.026 & 3.66 & 95 & 290.9 \\
\hline 15 & 109.31 & 10041210 & 0.187 & 0.937 & 0.009 & 255 & 194 & -5.1 & 0.038 & 1.88 & 96 & 294.9 \\
\hline 16 & 106.51 & 10091014 & 0.27 & 1.038 & 0.014 & 604 & 338 & -26.6 & 0.112 & 2.49 & 83 & 292.0 \\
\hline 17 & 101.31 & 9121610 & 0.161 & 0.624 & 0.029 & 177 & 156 & -7.7 & 0.026 & 1.86 & 92 & 268.8 \\
\hline 18 & 98.85 & 9050812 & 0.188 & 0.732 & 0.005 & 500 & 196 & -21.2 & 0.038 & 2.2 & 90 & 293.8 \\
\hline 19 & 97.42 & 10020111 & 0.186 & 1.309 & 0.005 & 550 & 193 & -4 & 0.026 & 1.98 & 92 & 270.9 \\
\hline 20 & 97.35 & 10022111 & 0.147 & 0.708 & 0.012 & 292 & 136 & -6.6 & 0.026 & 1.67 & 102 & 283.8 \\
\hline 21 & 96.51 & 8090308 & 0.178 & 1.04 & 0.009 & 324 & 180 & -4.1 & 0.112 & 1.28 & 73 & 299.9 \\
\hline 22 & 96.23 & 8091109 & 0.216 & 0.795 & 0.008 & 194 & 242 & -9.8 & 0.117 & 1.76 & 97 & 294.9 \\
\hline 23 & 96.00 & 9021311 & 0.216 & 1.131 & 0.005 & 443 & 241 & -7.7 & 0.026 & 2.49 & 89 & 279.2 \\
\hline 24 & 95.64 & 8072409 & 0.179 & 0.817 & 0.005 & 227 & 181 & -5.9 & 0.124 & 1.33 & 98 & 298.1 \\
\hline 25 & 91.64 & 9061307 & 0.184 & 0.798 & 0.005 & 228 & 189 & -6.9 & 0.124 & 1.4 & 93 & 293.8 \\
\hline
\end{tabular}

Table 20 Meteorological Conditions for Top 25 Modeled Concentrations at Gibson Tower Monitor Using Beta $u^{*}$ AERMET and SHARP

\begin{tabular}{|c|c|c|c|c|c|c|c|c|c|c|c|c|}
\hline Rank & Conc. & YYMMDDHH & $\mathrm{u}^{*}$ & $\mathbf{w}^{*}$ & VPTG & $z_{i c}$ & $z_{\text {im }}$ & $\mathbf{L}$ & $z_{0}$ & WSPD & WDIR & temp \\
\hline 1 & 218.99 & 10033010 & 0.16 & 0.715 & 0.006 & 179 & 154 & -5 & 0.026 & 1.76 & 310 & 285.9 \\
\hline 2 & 217.20 & 10021911 & 0.139 & 1.216 & 0.01 & 351 & 124 & -1.3 & 0.018 & 1.37 & 278 & 277.0 \\
\hline 3 & 186.74 & 9122112 & 0.113 & 0.535 & 0.005 & 307 & 91 & -7.2 & 0.019 & 1.38 & 307 & 274.9 \\
\hline 4 & 169.46 & 8081308 & 0.138 & 1.017 & 0.005 & 312 & 123 & -2 & 0.032 & 1.26 & 297 & 295.9 \\
\hline 5 & 165.06 & 8112811 & 0.143 & 0.967 & 0.013 & 365 & 130 & -3 & 0.019 & 1.57 & 304 & 280.4 \\
\hline 6 & 157.08 & 10030813 & 0.128 & 0.496 & 0.009 & 237 & 110 & -10.2 & 0.026 & 1.52 & 320 & 281.4 \\
\hline 7 & 143.69 & 10052609 & 0.152 & 1.57 & 0.005 & 646 & 143 & -1.5 & 0.026 & 1.4 & 324 & 301.4 \\
\hline 8 & 139.11 & 10081416 & 0.364 & 2.119 & 0.005 & 1790 & 527 & -22.7 & 0.047 & 4.09 & 303 & 309.9 \\
\hline 9 & 131.13 & 9072309 & 0.205 & 1.006 & 0.005 & 422 & 223 & -8.9 & 0.047 & 2.1 & 308 & 297.0 \\
\hline 10 & 130.84 & 8100111 & 0.406 & 1.809 & 0.009 & 873 & 621 & -24.7 & 0.041 & 4.73 & 300 & 290.9 \\
\hline 11 & 127.22 & 9061716 & 0.324 & 2.166 & 0.005 & 1704 & 444 & -14.3 & 0.032 & 3.8 & 287 & 305.9 \\
\hline 12 & 125.05 & 8102810 & 0.369 & 1.52 & 0.005 & 839 & 538 & -30 & 0.041 & 4.36 & 304 & 279.9 \\
\hline 13 & 123.18 & 9062014 & 0.309 & 1.889 & 0.005 & 1573 & 412 & -17.1 & 0.032 & 3.68 & 298 & 305.4 \\
\hline 14 & 123.03 & 9062817 & 0.495 & 2.168 & 0.012 & 2597 & 837 & -76.9 & 0.032 & 6.46 & 296 & 302.0 \\
\hline 15 & 122.31 & 10072909 & 0.241 & 1.108 & 0.005 & 393 & 283 & -10.1 & 0.047 & 2.5 & 310 & 302.5 \\
\hline 16 & 119.79 & 8022915 & 0.606 & 1.035 & 0.006 & 520 & 1131 & -260.8 & 0.018 & 9.06 & 294 & 287.0 \\
\hline 17 & 116.99 & 10020313 & 0.249 & 1.443 & 0.018 & 612 & 299 & -7.9 & 0.018 & 3.1 & 294 & 274.2 \\
\hline 18 & 115.05 & 9071615 & 0.297 & 1.447 & 0.007 & 993 & 388 & -21.4 & 0.047 & 3.32 & 303 & 303.1 \\
\hline 19 & 114.86 & 10060108 & 0.156 & 0.777 & 0.005 & 185 & 148 & -3.7 & 0.032 & 1.57 & 287 & 297.0 \\
\hline 20 & 113.39 & 8061711 & 0.388 & 1.956 & 0.013 & 1515 & 581 & -29.6 & 0.032 & 4.82 & 296 & 297.5 \\
\hline 21 & 111.80 & 10080612 & 0.321 & 2.341 & 0.007 & 1496 & 437 & -9.6 & 0.047 & 3.32 & 311 & 303.8 \\
\hline 22 & 111.59 & 9112110 & 0.104 & 0.939 & 0.007 & 265 & 81 & -1 & 0.025 & 0.89 & 261 & 286.4 \\
\hline 23 & 110.58 & 8102711 & 0.494 & 1.654 & 0.005 & 858 & 834 & -57.1 & 0.027 & 6.58 & 296 & 282.0 \\
\hline 24 & 109.28 & 10022613 & 0.434 & 1.797 & 0.011 & 920 & 687 & -32.4 & 0.019 & 5.99 & 301 & 277.0 \\
\hline 25 & 108.17 & 9020215 & 0.536 & 1.349 & 0.005 & 1146 & 943 & -179.7 & 0.019 & 7.89 & 302 & 276.4 \\
\hline
\end{tabular}




\title{
Evaluation of Low Wind Modeling Approaches for Two Tall-Stack Databases
}

\author{
Robert Paine, Olga Samani, and Mary Kaplan
}

AECOM, 250 Apollo Drive, Chelmsford, MA 01824

\section{Eladio Knipping and Naresh Kumar}

Electric Power Research Institute, 3420 Hillview Avenue, Palo Alto, CA 94304

\begin{abstract}
The performance of the AERMOD air dispersion model under low wind speed conditions, especially for applications with only one level of meteorological data and no direct turbulence measurements or vertical temperature gradient observations, is the focus of this study. The analysis documented in this paper addresses evaluations for low wind conditions involving tall stack releases for which multiple years of concurrent emissions, meteorological data, and monitoring data are available.
\end{abstract}

AERMOD was tested on two databases that had sub-hourly meteorological data available using several technical options: default mode, with various low wind speed beta options, and using the available sub-hourly meteorological data. These databases included: 1) Mercer County, a North Dakota database featuring five $\mathrm{SO}_{2}$ monitors in the vicinity of the Dakota Gasification Company's plant and the Antelope Valley Station power plant in an area of both flat and elevated terrain, and 2) a flat-terrain setting database with four $\mathrm{SO}_{2}$ monitors in the vicinity of Gibson Generating Station in southwest Indiana.

The low wind beta options show improvement in model performance helping to reduce some of the over-prediction biases currently present in AERMOD when run with regulatory default options. The overall findings with the low wind speed testing on these tall stack databases indicate that AERMOD low wind speed options have a minor effect for flat terrain locations, but can have a very significant effect for elevated terrain locations. The performance of AERMOD using low wind speed options leads to improved consistency of meteorological conditions associated with the highest observed and predicted concentration events. The available subhourly modeling results using the Sub-Hourly AERMOD Run Procedure (SHARP) are relatively 
unbiased and show that this approach should be seriously considered to address situations dominated by low-wind meander conditions.

\section{Introduction}

During low wind speed (LWS) conditions, the diffusion of pollutants is reducedlimited by the limited degree of fresh air dilution. Both monitoring observations and dispersion modeling results of this study indicate that high ground-level concentrations can occur in these conditions. Wind speeds less than $2 \mathrm{~m} / \mathrm{sec}^{-1}$ are generally considered to be "low", with steady-state modeling assumptions tending to be compromised at these low speeds (PasquillSmith et al., 1983).

Pasquill (see Van der Hoven, 1976) recognized that for such low wind speeds, a plume is unlikely to have any definable travel (Van der Hoven, 1976). Wilson et al. (1976) considered this wind speed $\left(2 \mathrm{~m} / \mathrm{sec}^{-1}\right)$ as the upper limit for conducting tracer experiments in low wind speed conditions.

Anfossi et al. (2005) noted that in LWS conditions, dispersion is characterized by meandering horizontal wind oscillations. They reportednoted that as the wind speed decreases, the standard deviation of the wind direction increases, making it more difficult to define a mean plume direction. Sagendorf and Dickson (1974) and Wilson et al. (1976) found that under LWS conditions, horizontal diffusion was enhanced because of this meander and the resulting groundlevel concentrations could be much lower than that predicted by steady-state Gaussian plume models that did not account for the meander effect.

A parameter that is used as part of the computation of the horizontal plume spreading in Environmental Protection Agency's (EPA's) preferred model,_AERMOD;, -(Cimorelli et al., $\underline{2005)}$ is the standard deviation of the crosswind component, $\sigma_{\underline{V}}$, which can be parameterized as being proportional to the friction velocity, $\mathbf{u}_{-}^{*}$ (Smedman, 1988; Mahrt, 1998). These investigators found that there was an elevated minimum value of $\sigma_{\mathrm{v}} \underline{\text { that was attributed to }}$ meandering. -While at higher wind speeds, small-scale turbulence is the main source of variance, lateral meandering motions appear to exist in all conditions. Hanna (1990) found that $\underline{\sigma}_{\underline{v}}$ maintains a minimum value of about $0.5 \mathrm{~m} / \mathrm{secs}^{-1}$ even as the wind speed approaches zero.

Hanna and Chowdhury et al. (2014) noted that a minimum sigma-v of $0.5 \mathrm{~m} / \mathrm{s}$ is a part of the formulation for the SCICHEM model(Chowdhury et al., 2014). 
Anfossi (2005) noted that meandering exists under all meteorological conditions regardless of the stability or wind speed, and this phenomenon sets a lower limit for the horizontal wind component variances as noted by Hanna (1990) over all types of terrain.

Another method to address wind meander was attempted by Sagendorf and Dickson (1974), who used a Gaussian model, but divided each computation period into sub-hourly (2-minute) time intervals and then summed the results to determine the total concentration. This approach directly addresses the wind meander during the course of an hour by using the sub-hourly wind direction for each period modeled. As we discuss below, this approach has some appeal, but the sub-hourly interval must not be so small as to distort the basis of the horizontal plume dispersion formulation. Since the horizontal dispersion shape function for stable conditions in AERMOD is formulated with parameterizations derived from the 10-minute release and sampling times of

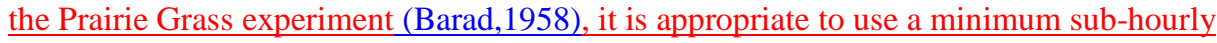
duration of 10 minutes for such modeling using AERMOD. - The Prairie Grass aspect of AERMOD may also result in an underestimate of the lateral plume spread in some cases, as reported by Irwin (2014) for Kincaid $\mathrm{SF}_{\underline{6}}$ releases. From analyses of hourly samples of $\mathrm{SF}_{\underline{6}}$ taken at Kincaid (a tall stack source), Irwin (2014) determined that the lateral dispersion simulated by AERMOD had a significant bias to underestimate the lateral dispersion (by 60\%) for near-stable conditions (conditions for which the lateral dispersion formulation that was fitted to the Project Prairie Grass data could affect results).

It is clear from the discussion above that the simulation of pollutant dispersion in LWS conditions is challenging. - In the United States, the use of steady-state plume models before the introduction of AERMOD in 2005 was done with the following rule implemented by the United States Envirenmental Protection AgencyEPA-(EPA, 68 FR 18440): "when used in steady-state Gaussian plume models, measured site-specific wind speeds of less than $1 \mathrm{~m} / \mathrm{sec}^{-1}$ but higher than the response threshold of the instrument should be input as $1 \mathrm{~m} / \mathrm{secs}^{-1}: "(\mathrm{EPA}, 2004)$.

With EPA's implementation of a new model, AERMOD, in 2005 (EPA, 200570 FR 68218), input wind speeds lower than $1 \mathrm{~m} / \mathrm{sec}^{-1}$ were permitted with the use of a meander algorithm that was designed to account for the LWS effects.- As noted in the AERMOD formulation document (EPA, 2004), 
"AERMOD accounts for meander by interpolating between two concentration limits: the coherent plume limit (which assumes that the wind direction is distributed about a well-defined mean direction with variations due solely to lateral turbulence) and the random plume limit, (which assumes an equal probability of any wind direction)."

A key aspect of this interpolation is the assignment of a time scale, TR, that is associated with a "time scale (=24 hours) at which mean wind information at the source is no longer correlated with the location of plume material at a downwind receptor." (EPA, 2004). The assumption of a full diurnal cycle for the e-folding period relating to this time scale tends to reduce the weighting of the random plume component relative to the coherent plume component for 1-hour time travel. -This weighting preference for the coherent plume leads to a minimization of the meander component, and a possible concentration overprediction. -As currently formulated, it is likely that the meander component in AERMOD is underestimated due to the time scale issue, and thus the coherent plume impact is overstated.

For conditions in which the plume is emitted aloft into a stable layer or in areas of inhomogenous terrain, it would be expected the decoupling of the stable boundary layer relative to the surface layer would significantly shorten this time scale. These effects are discussed by Brett and Tuller (1991), where they note that lower wind autocorrelations occur in areas with a variety of roughness and terrain effects. Perez et al. (2004) noted that the autocorrelation is reduced in areas with terrain and in any terrain setting with increasing height in stable conditions when decoupling of vertical motions would result in a loss of memory of surface conditions. Therefore, this study has reviewed the treatment of AERMOD in low wind conditions for field data involving terrain effects in stable conditions in contrast to flat terrain conditions, for which $\underline{\text { convective (daytime) conditions are typically associated with peak modeled predictions. }}$

The computation of the AERMOD coherent plume dispersion and the relative weighting of the

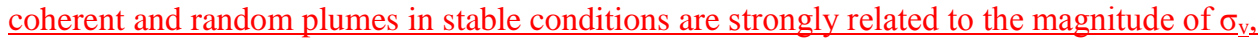
which is directly proportional to the magnitude of the friction velocity. Therefore, the formulation of the friction velocity calculation and the specification of a minimum $\sigma_{\underline{V}} \underline{v}$ value were also considered in this paper. The friction velocity also affects the internally-calculated vertical temperature gradient, which affects plume rise and plume-terrain interactions, which are especially important in elevated terrain situations. 
Qian and Venkatram (2011) discuss the challenges of LWS conditions in which the time scale of wind meandering is large, and the horizontal concentration distribution can be non-Gaussian.- It is also quite possible that wind instrumentation cannot adequately detect the turbulence levels that would be useful for modeling dispersion. They also noted that an analysis of data from the Cardington tower indicates that Monin-Obukhov similarity theory underestimates the surface friction velocity at low wind speeds. This finding was also noted by Paine et al. (2010) in an independent investigation of Cardington data as well as data from two other research-grade databases. Both Qian and Venkatram and Paine et al. proposed somewhat similar adjustments to the calculation of the surface friction velocity by AERMET, the meteorological processor for AERMOD. -EPA incorporated the Qian and Venkatram suggested approach as a "beta option" in AERMOD in late 2012 (EPA, 2012). - The same version of AERMOD-also introduced low wind modeling options that were used in the Test Cases 1-4 described below.

In its implementation of the 1 hour $\mathrm{SO}_{2}$ National Ambient Air Quality Standard (NAAQS), the United States Environmental Protection Agency (EPA) has been relying upon both dispersion modeling as well as traditional monitoring to assess attainment of the standard. The increased reliance upon modeling for determining NAAQS compliance for existing facilities is a new emphasis by EPA. In the spring of 2014, EPA released a proposed "Data Requirements Rule" (U.S.EPA, 2014a) that addresses many areas in the United States that have not been designated as to their attainment status with respect to the 1 hour $\mathrm{SO}_{2} \mathrm{NAAQS}$ that was promulgated (U.S. EPA, 2010) in 2010. As of March 2015, only 29 areas in 16 states with monitors showing NAAQS violations have been designated as to their attainment status (all nonattainment), while all other areas in the country have had their status deferred. This deferral has occurred because EPA regards $\mathrm{SO}_{2}$ as a unique pollutant with sharp concentration gradients and with peak impacts in the vicinity of relatively isolated industrial sources. For areas without monitoring data, but with large $\mathrm{SO}_{z}$-sources, the proposed Data Requirements Rule provides a procedure for determining the attainment status through either a dispersion modeling or monitoring approach.

Although this rule is directed at states, which need to act to determine the attainment status of the designated sources, the affected sources themselves are likely to be important stakeholders and may be asked by states to be involved in this process. The proposed rule provides both dispersion modeling and monitoring pathways to show attainment with the $S O_{z} \mathrm{NAAQS}$. While 
the modeling approach is the most cost-effective, the performance of dispersion modeling tools, such as EPA's preferred dispersion model for short range applications, AERMOD (Cimorelli et al., 2005), needs to be examined under a range of conditions that can influence attainment of a 1 hour standard. Specifieally, AERMOD's handling of low wind speed conditions, especially for applications with only one level of meteorological data and no direct turbulence measurements or vertical temperature gradient observations, is an isste of concern and is the focus of this study. Previous

Researchers have indicated that the treatment of low wind speeds by AERMOD is a key feature in its performance, especially since peak predictions often occur in low wind speed conditions (Paine et al., 2010). Past_evaluations of AERMOD for low wind speed conditions (e.g., Paine et al., 2010) haves emphasized low-level tracer release studies conducted in the 1970s and have utilized results of researchers such as Luhar and Rayner (2009). The focus of the study reported here is a further evaluation of AERMOD for tall-stack field databases. One of these databases was previously evaluated (Kaplan et al., 2012) with AERMOD Version 12345, featuring a database in Mercer County, North Dakota. This database features five $\mathrm{SO}_{2}$ monitors in the vicinity of the Dakota Gasification Company's plant and the Antelope Valley Station power plant in an area of both flat and elevated terrain. In addition to the Mercer County, ND database, this study considers an additional field database for the Gibson Generating Station tall stack in flat terrain in southwest Indiana.

EPA released AERMOD version 14134 en May 16, 2014-with enhanced low wind model features that can be applied in more than one combination. There is one low wind option (beta $\mathrm{u}_{*}$ ) applicable to the meteorological pre-processor, AERMET, affecting the friction velocity calculation, and a variety of options available for the dispersion model, AERMOD, that focus upon the minimum $\underline{\sigma}_{\text {sigma-v }}$ specification. Sigma $v$ is the standard deviation of the cresswind component of the horizontal wind speed; it is proportional to the product of the wind direction meander and the horizontal wind speed. These beta options have the potential to reduce the over-prediction biases currently present in AERMOD when run for neutral to stable conditions with regulatory default options (U.S-EPA, 2014 ba,eb). These new low wind options in AERMET and AERMOD are currently non-default options and thus require additional justification for each application in order to be considered for use in the United StatesSAThese beta options show a considerable improvement in model performance that help to reduce some of 
the over-prediction biases currently present in AERMOD when run with regulatory default options (U.S. EPA, 2014 b, c). However, the new low wind options in AERMET and AERMOD are currently non-default options and thus require additional justification for each application in order to be considered for use. . While EPA has conducted evaluations on low-level, nonbuoyant studies with the AERMET and AERMOD low wind speed beta options, they have not conducted any new evaluations on tall stack releases (U.S. EPA, 2014 ba,$\in \underline{b}$ ). One of the purposes of this study wasis to augment the evaluation experiences for the low wind model approaches for a variety of settings for tall stack releases.

This study also made use of the availability of sub-hourly meteorological observations to evaluate another modeling approach. This approach employs AERMOD with sub-hourly meteorological data and is known as the "Sub-Hourly AERMOD Run Procedure" or SHARP (EPRI, 2013). Like the procedure developed by Sagendorf and Dickson as described above, SHARP merely subdivides each hour's meteorology into equal parts (e.g., six 10-minute periods) and AERMOD is run sixmultiple times with the meteorological input data (e.g., minutes 1-10, 11-20, etc.) treated as hourly averages for each run. -Then, the results of these-multiple runs- 6 in this example)-are averaged. In our SHARP runs, we used no observed turbulence data as input. This alternative modeling approach (our Test Case 5 as discussed below) has been compared to the AERMOD default and low wind modeling approaches (Test Cases 1-4 described below, using hourly averaged meteorological data) to determine whether it should be further considered as a viable technique. This study provides a discussion of the various low wind speed modeling options and the field study databases that were tested, as well as the modeling results.

\section{Modeling Options and Databases for Testing}

Five AERMET/AERMOD model configurations were tested, as listed below. All model applications used one wind level, a minimum wind speed of $0.5 \mathrm{~m} / \mathrm{s}^{-1} \mathrm{~m} / \mathrm{sec}$, and also used hourly average meteorological data with the exception of SHARP applications. As noted above, Test Cases 1-4 used available options in the current AERMOD code. The selections for Test Cases 1-4 exercise low wind speed options over a range of reasonable choices extended from no low 


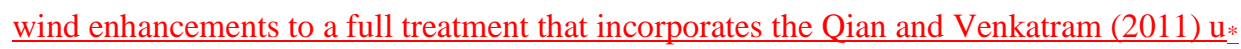

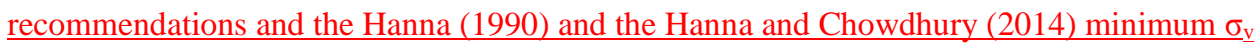
recommendations. Test Case 5 used sub-hourly meteorological data, but AERMOD was other run in a mode similar to Test Case 2 for SHARP applications. We discuss later in this document our recommendations for SHARP modeling without the AERMOD meander component included.

Test Case 1: AERMET and AERMOD in default mode.

Test Case 2: Low wind beta option for AERMET and default options for AERMOD (minimum $\underline{\sigma}_{\underline{V}}$ sigma- $\mathrm{V}$-value of $0.2 \mathrm{~m} / \mathrm{s}^{-1} \mathrm{~m} / \mathrm{sec}$ ).

Test Case 3: Low wind beta option for AERMET and the LOWWIND2 option for AERMOD (minimum $\underline{\sigma}_{-}$sigma- $₹$ value of $0.3 \mathrm{~m} / \mathrm{s}^{-1} \mathrm{~m} / \mathrm{sec}$ ).

Test Case 4: Low wind beta option for AERMET and the LOWWIND2 option for AERMOD (minimum $\underline{\sigma}_{\underline{V}}$ sigma- $v$-value of $0.5 \mathrm{~m} / \mathrm{s}^{-1} \mathrm{~m} / \mathrm{sec}$ ).

Test Case 5: Low wind beta option for AERMET and AERMOD run in sub-hourly mode (SHARP) with default low wind options.

\footnotetext{
An elevated minimum sigma- $v$ value, especially in the presence of elevated terrain, is consistent with the understanding that terrain influences will increase the plume dispersion near hills. This feature is present in the EPA approved CTDMPLUS model (U.S. EPA, 1989), but AERMOP has no capability to locally increase the plume horizontal dispersion near terrain. For example, the CTDMPLUS user manual indicates that "typically, the plume stretches in the horizontal as it passes over the crest of a simple three-dimensional hill, and this stretching produces a wider 'footprint' over the hill" (U.S. EPA, 1989). In a similar manner, the CTDMPLUS plume "wraps" around a hill if it passes below the dividing streamline height, which also locally increases the plume horizontal dispersion. This increase in the plume dispersion is not able to be locally induced in AERMOD near terrain features, but the use of an increase in the minimum sigma $v$ in stable conditions gees in the right direction toward simulating this effect. This elevated minimum sigma- $v$ value in stable conditions would not be expected to have much of an effect on the performance of $A E R M O D$ in flat terrain receptors.
} 


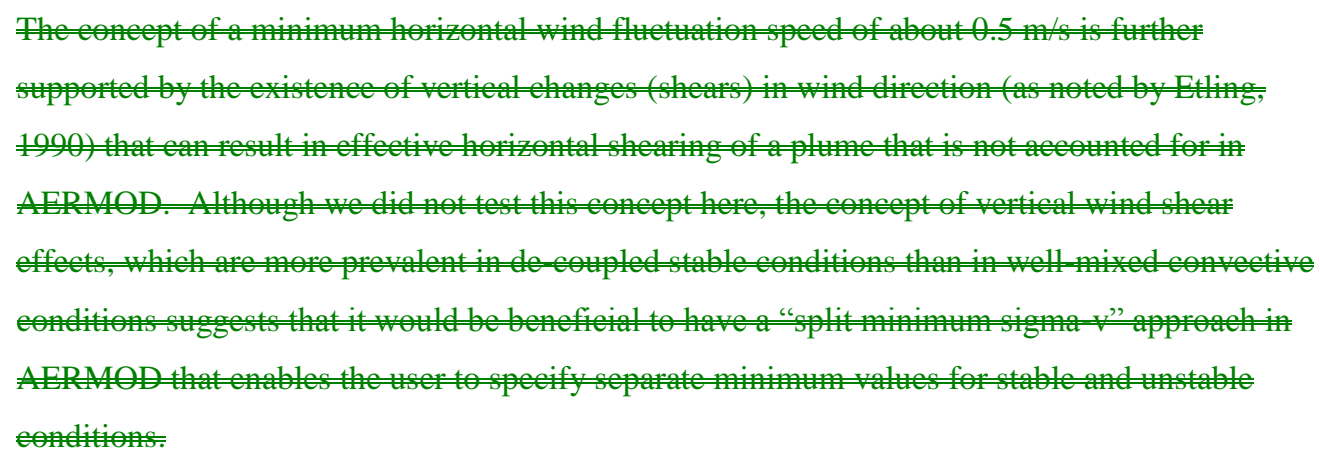

The databases that were selected for the low wind model evaluation are listed in Table 1 and described below. They were selected due to the following attributes:

- They feature multiple years of hourly $\mathrm{SO}_{2}$ monitoring at several sites.

- Emissions are dominated by tall stack sources that are mostly available from continuous emission monitors.

- They include sub-hourly meteorological data so that the SHARP model could be tested as well.

- $\quad$ There is representative meteorological data from a single-level station typical of (or obtained from) airport-type data.

Table 1 here.

\section{1) Mercer County, North Dakota}

An availableThe 4-year period of 2007-2010 was used for the Mercer County, ND database with five $\mathrm{SO}_{2}$ monitors within $10 \mathrm{~km}$ of two nearby emission facilities (Antelope Valley and Dakota Gasification Company), site-specific meteorological data at the DGC \#12 site (10-m level data in a low-cut grassy field in the location shown in Figure 1), and hourly emissions data from 15 point sources. The terrain in the area is rolling and features three of thefour monitors (Beulah,

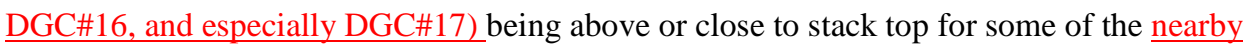
emission sources; see Figure 2 for more details. Figure 1 shows a layout of the sources, and monitors, and the meteorological station. Tables 2 and 3 provide details about the emission sources and the monitors. Although this modeling application employed sources as far away as $\underline{50 \mathrm{~km} \text {, the proximity of the monitors to the two nearby emission facilities implies that emissions }}$ 
from those facilities dominated the impacts. -However, to avoid criticism that other regional sources that could have been modeled were omitted, the other lignite-fired power plants were included in the modeling.

2) Gibson Generating Station, Indiana

An availableThe 3-year period of 2008-2010 was used for the Gibson Generating Station in southwest Indiana with four $\mathrm{SO}_{2}$ monitors within $6 \mathrm{~km}$ of the plant, airport hourly meteorological data (from Evansville, IAndiana 1-minute data, located about $40 \mathrm{~km}$ SSE of the plant), and hourly emissions data from one electrical generating station (Gibson). The terrain in the area is quite flat and the stacks are tall. Figure $\underline{3} z$ depicts the locations of the emission source and the four $\mathrm{SO}_{2}$ monitors. Although the plant had an on-site meteorological tower, EPA (2013) noted that the tower's location next to a large lake resulted in non-representative winds for the $\underline{\text { area, and that the use of airport data would be preferred. Tables } 2 \text { and } 3 \text { provide details about the }}$ $\underline{\text { emission sources and the monitors. Due to the fact that there are no major } \mathrm{SO}_{2}} \underline{\text { sources within at }}$ least $30 \mathrm{~km}$ of Gibson, we modeled emissions from only that plant.

Figure 1 here.

Figure 2 here.

Figure 3 here.

\section{Meteorological Data Processing}

For the North Dakota and Gibson database evaluations, the hourly surface meteorological data were processed with AERMET, the meteorological preprocessor for AERMOD. The boundary layer parameters were developed according to the guidance provided by EPA in the current AERMOD Implementation Guide (U.S.EPA, 2009). For the first modeling evaluation option, Test Case 1, AERMET was run using the default options. For the other four model evaluation options, Test Case 1 to 4, AERMET was run with the beta $\mathrm{u}_{*}$ low wind speed option.

\section{North Dakota Meteorological Processing}

Four years (2007-2010) of the 10-meter meteorological data collected at the DGC\#12 monitoring station (located about $7 \mathrm{~km} \mathrm{SSE}$ of the central emission sources) were processed with 
AERMET. The data measured at this monitoring station were wind direction, wind speed, and temperature. Hourly cloud cover data from the Dickinson Theodore Roosevelt Regional Airport, North Dakota (KDIK) ASOS station $(85 \mathrm{~km}$ to the SW) were used in conjunction with the monitoring station data. Upper air data were obtained from the Bismarck Airport, North Dakota (KBIS; about $100 \mathrm{~km}$ to the SE), twice-daily soundings.

In addition, the sub-hourly (10-minute average) 10-meter meteorological data collected at the DGC\#\#12 monitoring station were also processed with AERMET. AERMET was set up to read six 10-minute average files with the tower data and output six 10-minute average surface and profile files for use in SHARP. SHARP then used the sub-hourly output of AERMET to calculate hourly modeled concentrations, without changing the internal computations of AERMOD. The SHARP user's manual (EPRI, 2013) provides detailed instructions on processing sub-hourly meteorological data and executing SHARP.

\section{Gibson Meteorological Processing}

Three years (2008-2010) of hourly surface data from the Evansville Airport, Indiana (KEVV) ASOS station (about $40 \mathrm{~km}$ SSE of Gibson) were used in conjunction with the twice-daily soundings upper air data from the Lincoln Airport, Illinois (KILX, about $240 \mathrm{~km} \mathrm{NW}$ of Gibson). The 10-minute sub-hourly data for SHARP were generated from the 1-minute meteorological data collected at Evansville Airport.

\section{Emission Source Characteristics}

Table 2 summarizes the stack parameters and locations of the modeled sources for the North Dakota and Gibson databases. Actual hourly emission rates, stack temperatures, and stack gas exit velocities were used for both databases.

Table 2 here.

\section{Model Runs and Processing}


For each evaluation database, the candidate model configurations were run with hourly emission rates provided by the plant operators reflective of the best estimates of the actual emissions for each hour of the study. In the case of rapidly-varying emissions (startup and shutdown), the hourly averages may average intermittent conditions during the hour. Actual stack heights were used, along with building dimensions used as input to the models tested. Receptors were placed only at the location of each monitor to match the number of observed and predicted

concentrations. While it is likely that modeled peak impacts for some hours could be located in the vicinity of monitors (but not exactly at the monitor location), only the predictions at the actual location of each monitor were considered for comparison to the observations at that monitor.

SHARP was run on the North Dakota and Gibson databases as they both had sub hourly meteorological data. The SHARP user's manual provides detailed instructions on postprocessing multiple AERMOD runs and creating a single output file.

The monitor (receptor) locations and elevations are listed in Table 3. For the North Dakota database, the DGC\#17 monitor is located in elevated terrain. The monitors for the Gibson database wereare located at elevations at or near stack base, with stack heights ranging from 152 to 189 meterswell below the Gibson stack tops.

Table 3 here.

\section{Tolerance Range for Modeling Results}

One issue to be aware of regarding $\mathrm{SO}_{2}$ monitored observations is that they can exhibit over- or under-prediction tendencies up to $10 \%$ and still be acceptable. This is related to the tolerance in the EPA procedures (U.S.EPA, 2013) associated with quality control checks and span checks of ambient measurements. Therefore, even ignoring uncertainties in model input parameters and other contributions (e.g., model science errors and random variations) that can also lead to modeling uncertainties, just the uncertainty in measurements indicate that modeled-to-monitored ratios between 0.9 and 1.1 can be considered as "unbiased" within an unbiased range. In the discussion below, we consider model performance to be "relatively unbiased" if its predicted

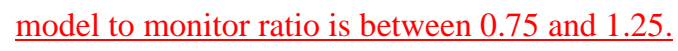




\section{Model Evaluation Metrics}

The model evaluation employed metrics that address threefeur basic areas, as described below.

\section{1) 1-hour $\mathrm{SO}_{2}$ NAAQS Design Concentration}

An operational metric that is tied to the form of the 1-hour $\mathrm{SO}_{2} \mathrm{NAAQS}$ is the "design concentration" ( $99^{\text {th }}$ percentile of the peak daily 1 -hour maximum values). This tabulated statistic was developed for each modeled case and for each individual monitor for each database evaluated. Results for this statistic are included in the discussion below.

2) Time Series Plots

Time series plots of the observed and predicted daily maximum 1 hour $\mathrm{SO}_{z}$-concentrations were also developed. While the tabulation of the design concentration provides a comparison of just one value for the predictions and observations, the time series plot provides a comparison for the entire period evaluated. The plots show the relative frequency and magnitudes of the eoncentration predictions and observations. Review of these plots results is somewhat of a qualitative, but informative, finding regarding the performance of each model and also presents seasenal distributions of the concentration patterns for both observations and predictions. These plots were considered in the evaluation and can be found in the supplemental files.

\section{3)2)_Quantile-Quantile Plots}

Operational performance of models for predicting compliance with air quality regulations, especially those involving a peak or near-peak value at some unspecified time and location, can be assessed with quantile-quantile (Q-Q) plots (Chambers et al., 1983), which are widely used in AERMOD evaluations. Q-Q plots are created by independently ranking (from largest to smallest) the predicted and the observed concentrations from a set of predictions initially paired in time and space. Quantile quantile $(Q Q)$ plots of the ranked daily maximum 1 hour $\mathrm{SO}_{2}$ eoncentrations for predictions and observations are useful. A robust model would have all points on the diagonal (45-degree) line. The sorted list of predicted concentrations is then plotted by rank against the observed concentrations alse sorted by rank. These concentration pairs are no longer paired in time, but we have retained the location pairing in this evaluation study. Such plots are useful for answering the question, "Over a period of time evaluated, does the distribution of the model predictions match those of observations?" Scatterplots, which use data paired in time, would provide a stricter test, answering the question: "At a given time and place, does the magnitude of the model prediction match the observation?" However, when modeling 
the impact of sources at short ranges, the impact of even minor wind-direction uncertainties can be quite large and cause even well-formulated dispersion models to appear to perform inadequately (Weil et al., 1992; Lim et al., 1984). Therefore, the Q-Q plot instead of the scatterplot is a pragmatic procedure for demonstrating model performance of applied models,

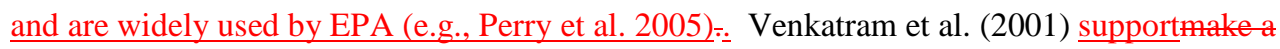
eogent argument for the use of Q-Q plots for evaluating regulatory models. Quantile-quantile (Q-Q) plots of the ranked daily maximum 1 hour $S \theta_{2}$ concentrations for predictions and ebservations are useful. A robust model would have all points on the diagonal (45-degree) line. Several Q-Q plots are included in this paper in the discussion provided below.

4) 3)_Meteorological Conditions Associated with Peak Observed vs Modeled Concentrations

Lists of the meteorological conditions and hours/dates of the top several 25 predictions and observations provide an indication as to whether these conditions are consistent between the model and monitoring data. For example, if the peak observed concentrations generally occur during daytime hours, we would expect that a well-performing model would indicate that the peak predictions are during the daytime as well. Another meteorological variable of interest is the wind speed magnitudes associated with observations and predictions. It would be expected, for example, that if the wind speeds associated with peak observations are low, then the modeled peak predicted hours would have the same characteristics. A brief qualitative summary of this analysis is included in this paper and supplemental files contain the tables of the top 25 (unpaired) predictions and observations for all monitors and cases tested.

The United States EPA has a statistical evaluation software package, the Model Evaluation Methodology (Strimaitis et al., 1993), which can provide confidence intervals for the model eomparisons of the type conducted in this study. This approach uses the Robust Highest Concentration metric diseussed by EPA ("Protocol for Determining the Best Performing Model", 1992). However, the software is outmoded in that it does not consider meteorologicat parameters used by AERMOD (it uses the older stability class approach) and it does not consider the new form of the USA SOz ambient standard $\left(99^{\text {th }}\right.$ percentile peak daily 1 hour maximum). Therefore, to estimate the likelihood that certain model performance differences reflected significant improvements, we refer below to other related work that provides an indication of this metric as a function of comparative model over-prediction or under-prediction bias. 


\section{North Dakota Database Model Evaluation Procedures and Results}

AERMOD was run for five test cases to compute the 1-hour daily maximum $99^{\text {th }}$ percentile averaged over four years at the five ambient monitoring locations listed in Table 3.

A regional background of $10 \mu \mathrm{g} / \mathrm{m}^{3}$ was added to the AERMOD modeled predictions. $;$ Tthe 1hour $99^{\text {th }}$ percentile background concentration was computed from the 2007-2010 lowest hourly monitored concentration among the five monitors so as to avoid double-counting impacts from sources already being modeled.

The ratios of the modeled (including the background of $10 \mu \mathrm{g} / \mathrm{m}^{3}$ ) to monitored design concentrations are summarized in Table 4 and graphically plotted in Figure $\underline{z} z$ and are generally greater than 1. (Note that the background concentration is a small fraction of the total concentration, as shown in Table 4). For the monitors in simple terrain (DGC\#12, DGC\#14, and Beulah), the evaluation results are similar for both the default and beta options and are within $5-30 \%$ of the monitored concentrations depending on the model option. The evaluation result for the monitor in the highest terrain (DGC-\#17) shows that the ratio of modeled to monitored concentration is over 2, but when this location is modeled with the AERMET and AERMOD low wind beta option, the ratio is significantlynotably better, at less than 1.3. It is noteworthy that the modeling results for inclusion of just the beta $\mathrm{u} *$ option are virtually identical to the default AERMET run for the simple terrain monitors, but the differences are significant for the higher terrain monitor (DGC-\#17). For all of the monitors, it is evident that further reductions of AERMOD's over-predictions occur as the minimum $\underline{\sigma}_{\underline{V}}$ sigma- $\vee$ in AERMOD is increased from 0.3 to $0.5 \mathrm{~m} / \mathrm{s}^{-1} \mathrm{~m} / \mathrm{sec}$. Even for a minimum $\underline{\sigma_{\underline{ }}}$ sigma- $v$ of $0.5 \mathrm{~ms}^{-1} \mathrm{~m} / \mathrm{sec} / \mathrm{s}$ at all the monitors, AERMOD is shown to be conservative with respect to the design concentration.

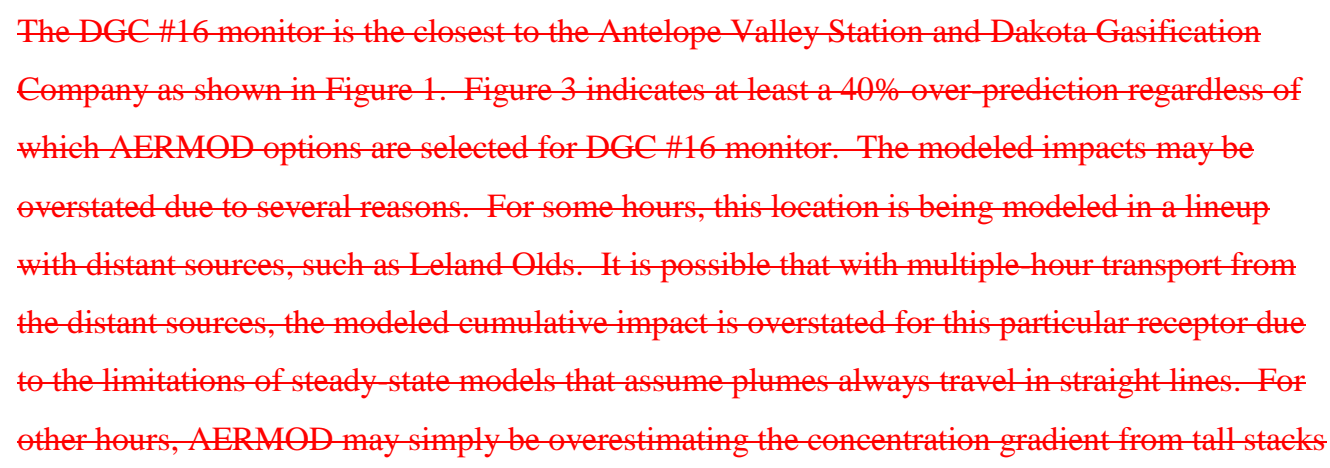


with buoyant emissions such as Antelope Valley Station and Dakota Gasification Company. It is also possible that for hours with transient start up or shutdown conditions, the hourly averaged model input for the exhaust parameters leads to model over predictions. In such a case, the plume rise could vary substantially during the hour, but the models cannot simulate this without sub-hourly stack exhaust data, which is not available.

The Q-Q plots of the ranked top fifty daily maximum 1-hour $\mathrm{SO}_{2}$ concentrations for predictions and observations are shown in Figure 5 F. For the convenience of the reader, a vertical dashed line is included in each Q-Q plot to indicate the observed design concentration. In general, the Q-Q plots indicate the following:

- For all of the monitors, to the left of the design concentration line, the AERMOD hourly runs all show ranked predictions at or higher than observations. To the right of the design concentration line, the ranked modeled values for specific test cases and monitors are sometimes-lower than the ranked observed levels, and the slope of the line formed by the plotted points is less than the slope of the 1:1 line. For model performance goals that would need to predict well for the peak concentrations (rather than the $99^{\text {th }}$ percentile statistic), this area of the Q-Q plots would be of greater importance.

- The very highest observed value (if indeed valid) is not matched by any of the models for all of the monitors, but since the focus is on the $99^{\text {th }}$ percentile form of the United States ambient standard for $\mathrm{SO}_{2}$, this area of model performance is not important for this application.

- The ranked SHARP modeling results are lower than all of the hourly AERMOD runs, but at the design concentration $\left(99^{\text {th }}\right.$ percentile $)$ level, they are, on average, relativelynearly unbiased over all of the monitors. The AERMOD runs for SHARP included the meander component, which probably contributed to the small under-predictions noted for SHARP. In future modeling, we would advise users of SHARP to employ the AERMOD LOWWIND1 option to disable the meander component.

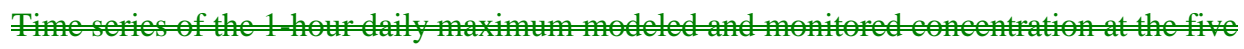
monitor loeations were investigated and provided in the supplemental file called "Nonth Dakota Time Series Plots". The allo the day tested. General onelusions from the review the ofor follows: 
Peak strong winds. A few peak concentrations oceur at night with light winds.

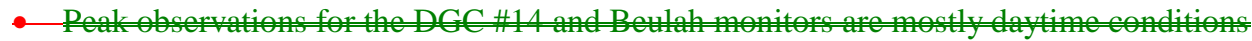
with a large range of wind speeds. Some peak concentrations oceur at night with a large of

- Peak observations for the DGC \#16 and DGC \#17 monitors are mixed be day and nighttime conditions with a large range of wind speeds for both.

The conclusions from the review of the meteorologieal conditions associated with peak

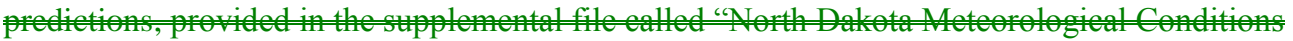
Resulting in Top 25 Concentrations", areas follows:

- AERMOQ huly

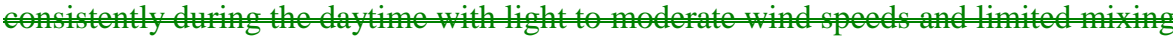
heights.

- There is a similar AERMOD result for DGC \#14, excep that there are more periods with high winds and higher mixing heights.

- The AERMOD result for DGC \#16 still features mostly daytime hours, but with more hing

- - The default AERMOD results fOr DGC \#17 are distinetly different, with most hours featuring stable, light winds. There are a few daytime hours with low winds and low mixing heights. This patten changes substantially with the betaleoptions employed, when the majority of the peak prediction hours are daytime periods with light to moderate wind speeds. This pattern is more consistent with the peak obsed concentration enditions,

- The SHARP peak predictions were also mostly associated with daytime hours with a large range of wind speeds for all of the menitors.

Table 4 here.

Figure 43 here.

Figure $\underline{5} 4$ here. 


\section{Gibson Generating Station Database Model Evaluation Procedures and Results}

AERMOD was run for five test cases to compute the 1-hour daily maximum $99^{\text {th }}$ percentile averaged over three years at the four ambient monitoring locations listed in Table 3.

A regional background of $18 \mu \mathrm{g} / \mathrm{m}^{3}$ was added to the AERMOD modeled predictions $;$. $€$ The 1hour $99^{\text {th }}$ percentile background concentration was computed from the 2008-2010 lowest hourly monitored concentration among the four monitors so as to avoid impacts from sources being modeled.

The ratio of the modeled (including the background of $18 \mu \mathrm{g} / \mathrm{m}^{3}$ ) to monitored concentrations isare summarized in Table 5 and graphically plotted in Figure $\underline{6} 4$ and are generally greater than 1.0. (Note that the background concentration is a small fraction of the total concentration, as shown in Table 5.) Figure 64 shows that AERMOD with hourly averaged meteorological data over-predicts by about 40-50\% at Mt. Carmel and Gibson Tower monitors and by about 9-31\% at East Mt. Carmel and Shrodt monitors. As expected (due to dominance of impacts with convective conditions), the AERMOD results do not vary much with the various low wind speed options in this flat terrain setting. AERMOD with sub-hourly meteorological data (SHARP) has the best (least biased predicted-to-observed ratio of design concentrations) performance among the five cases modeled. Over the four monitors, the range of predicted-to-observed ratios for SHARP is a narrow one, ranging from a slight under-prediction by $2 \%$ to an over-prediction by $14 \%$.

The Q-Q plots of the ranked top fifty daily maximum 1-hour $\mathrm{SO}_{2}$ concentrations for predictions and observations are shown in Figure 76. It is clear from these plots that the SHARP results parallel and are closer to the 1:1 line for a larger portion of the concentration range than any other model tested. In general, AERMOD modeling with hourly data exhibits an over-prediction tendency at all of the monitors for the peak ranked concentrations at most of the monitors. The AERMOD/SHARP models predicted lower relative to observations at the East Mt. Carmel monitor for the very highest values, but match well for the $99^{\text {th }}$ percentile peak daily 1-hour maximum statistic.

Time series of the 1 hour daily maximum modeled and monitered concentration at the four monitor locations were investigated and provided in the supplemental file called "Gibson Time 
Series Plots". The conclusions from the review of the meteorological conditions associated with peak predictions, provided in the supplemental file called "Gibson Meteorological Conditions Resulting in Top 25 Concentrations", are as follows:

- Peak observations for the Mt. Carmel and East Mt. Carmel monitors occur during both light wind convective conditions and strong wind conditions (near neutral, both daytime and nighttime).

- The Gibson Tower monitor peak observation conditions were similarly mixed for wind speeds, but they were consistently occurring during the daytime only.

- In contrast, peak observations for the Shrodt monitor are consistently associated with convective (daytime) conditions and low winds.

- Nighttime peaks that are noted at Mt. Carmel and East Mt. Carmel could be due to downwash effects with southerly winds. Gibsen Tower and Schrodt monitors were in directions with minimal downwash effects, therefore, the peak impacts at these monitors eccur with convective conditions.

AERMOD (hourly) modeling runs and SHARP runs are generally consistent with the patterns of observed conditions for all of the monitors.

Table 5 here.

Figure $\underline{6} 5$ here.

Figure 76 here.

\section{Evaluation Results Discussion}

The modeling results for these tall stack releases are sensitive to the source local setting and proximity to complex terrain. In general, for tall stacks in simple terrain, the peak ground-level impacts mostly occur in daytime convective conditions. For settings with a mixture of simple and complex terrain, the peak impacts for the higher terrain are observed to occur during both daytime and nighttime conditions, while AERMOD tends to favor stable conditions only without low wind speed enhancements. Exceptions to this "rule of thumb" can occur for stacks with aerodynamic building downwash effects. In that case, high observed and modeled predictions $\underline{\text { can occur during high wind events. }}$ 
The significance of the changes in model performance (using a $90^{\text {th }}$ percentile confidence interval) was independently tested for a similar model evaluation conducted for Eastman

Chemical Company (Paine et al., 2013; Szembek et al., 2013), using a modification of the Model Evaluation Methodology (MEM) MEM software that computed estimates of the hourly stability class (Strimaitis et al., 1993).- That study indicated that relative to a perfect model, a model that over-predicted or under-predicted by less than about $50 \%$ would likely show a performance level that was not significantly different. For a larger difference in bias, one could expect a statistically significant difference in model performance.

A review of the North Dakota ratios of monitored to modeled values in Figure 4 generally indicates that for DGC\#12, DGC\#14, and Beulah, the model differences were all not significantly different. For DGC\#16, it could be concluded that the SHARP results were significantly better than the default AERMOD results, but other AERMOD variations were not significantly better. For the high terrain monitor, DGC\#17, is it evident that all of the model options departing from default were significantly better than the default option, especially the SHARP approach.

For the Gibson monitors (see Figure 6), the model variations did not result in significantly different performance except for the Gibson Tower (SHARP vs. the hourly modes of running AERMOD).

Time series of the 1 hour daily maximum modeled and monitored concentration at the five monitor locations were investigated and provided in the supplemental file called "North Dakota Time Series Plots". They are useful supporting information because they present data over all of the days tested. General conclusions from the review of meteorological conditions associated with the top observed concentrations at the North Dakota monitors, provided in the supplemental file called "North Dakota Meteorological Conditions Resulting in Top 25 Concentrations", are as follows:

- Some peak oserved concentrations occur at night with light winds. Majority of Peak observations for the DGC-\#12 monitor are mostly daytime conditions with moderate to strong winds. A minority of the few peak observed concentrations occur at night with light winds. 
- Peak observations for the DGC\#14 and Beulah monitors are mostly daytime conditions with a large range of wind speeds. Once again, a minority of the $\underline{\underline{\text { Some peak }}}$ concentrations occur at night with a large range of wind speeds.

- Peak observationsed concentrations for the DGC\#16 and DGC\#17 monitors occur at night with light winds. Majority of observations are mixed between daytime and nighttime conditions with a large range of wind speeds for both. The DGC\#17 monitor is located in elevated terrain.

The conclusions from the review of the meteorological conditions associated with peak AERMOD or SHARP predictions, provided in the supplemental file called "North Dakota Meteorological Conditions Resulting in Top 25 Concentrations", are as follows:

- AERMOD hourly peak predictions for the DGC\#12 and Beulah monitors are consistently during the daytime with light to moderate wind speeds and limited mixing heights. This is a commonly observed situation that is further discussed below.

- There are is a similar AERMOD results for DGC\#14, except that there are more periods with high winds and higher mixing heights.

- The AERMOD results for DGC\#16 still features mostly daytime hours, but with more high wind conditions.

- The default AERMOD results for DGC\#17 are distinctly different from the other monitors, with most hours featuring stable, light winds. There are also a few daytime hours of high predictions with low winds and low mixing heights. This pattern changes substantially with the beta $\underline{\underline{u}} *$ options employed, when the majority of the peak prediction hours are daytime periods with light to moderate wind speeds. This pattern is more consistent with the peak observed concentration conditions.

- The SHARP peak predictions at the North Dakota monitors were also mostly associated with daytime hours with a large range of wind speeds for all of the monitors.

The North Dakota site has some similarities due to a mixture of flat and elevated terrain to the Eastman Chemical Company model evaluation study in Kingsport, TTennesseeN (this site features three coal-fired boiler houses with tall stacks). - In that study (Paine et al. 2013; Szembek et al., 2013), there was one monitor in elevated terrain and two monitors in flat terrain with a full year of data. Both the North Dakota and Eastman sites featured observations of the 
$99^{\text {th }}$ percentile peak daily 1 hour (“design”) concentration being within about $10 \%$ of the mean design concentration over all monitors.- Modeling results using default options in AERMOD for both of these sites indicated a large spread of the predictions, with predictions in high terrain exceeding observations by more than a factor of 2 . - In contrast, the predictions in flat terrain, while higher than observations, showed a lower overprediction bias. The use of low wind speed improvements in AERMOD (beta u $*$ in AERMET and an elevated minimum $\sigma_{v}$ value) did improve model predictions for both databases.

The conclusions from the review of the meteorological conditions associated with peak observations predictions, provided in the supplemental file called "Gibson Meteorological Conditions Resulting in Top 25 Concentrations", are as follows:

- - Peak observations for the Mt. Carmel and East Mt. Carmel monitors occur during both $\underline{\text { light wind convective conditions and strong wind conditions (near neutral, both daytime }}$ and nighttime). Nighttime peaks that are noted at Mt. Carmel and East Mt. Carmel could be due to downwash effects with southerly winds. Gibson Tower and Schrodt monitors were in directions with minimal downwash effects, therefore, the peak impacts at these monitors occur with convective conditions.

- The Gibson Tower and Shrodt monitor peak observation conditions were similarly mixed for wind speeds, but they were consistently occurring during the daytime only.

- Peak observations for the Shrodt monitor are consistently associated with eonvective (daytime) conditions and low winds.

- Nighttime peaks that are noted at Mt. Carmel and East Mt. Carmel could be due

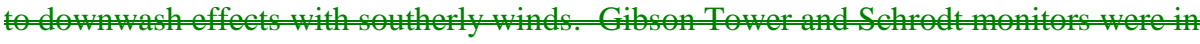

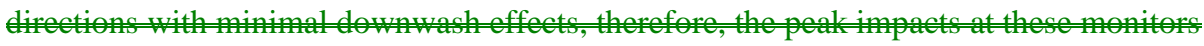
dition

AERMOD (hourly) modeling runs and SHARP runs are generally consistent with the patterns of observed conditions for Shrodt and Gibson Tower all of the-monitors. Except for downwash effects, the peak concentrations were all observed and predicted during daytime hours. There are $\underline{\text { similar AERMOD results for Mt. Carmel and East Mt. Carmel, except that there are more }}$ nighttime periods and periods with strong wind conditions. 
As noted above, AERMOD tends to focus its peak predictions for tall stacks in simple terrain (those not affected by building downwash) for conditions with low mixing heights in the morning. However, a more detailed review of these conditions indicates that the high predictions are not simply due to plumes trapped within the convective mixed layer, but instead due to plumes that initially penetrate the mixing layer, but then emerge (after a short travel time) in the convective boundary layer in concentrated form with a larger-than-expected vertical spread. Tests of this condition were undertaken by Dr. Ken Rayner of the Western Australia Department of Environmental Regulation (2013), who found the same condition occurring for tall stacks in simple terrain for a field study database in his province. Rayner found that

AERMOD tended to over-predict peak concentrations by a factor of about $50 \%$ at a key monitor, while with the penetrated plume removed from consideration, AERMOD would underpredict by about 30\%. Therefore, the correct treatment might be a more delayed entrainment of the penetrated plume into the convective mixed layer. Rayner's basic conclusions were that:

- A plume penetrates and disperses within a 1-hour time step in AERMOD, while in the real world, dispersion of a penetrated puff may occur an hour or more later, after substantial travel time.

- A penetrated plume initially disperses via a vertical Gaussian formula, not a convective probability density function.- Because penetrated puffs typically have a very small vertical dispersion, they are typically fully entrained (in AERMOD) in a single hour by a growing mixed layer, and dispersion of a fully entrained puff is via convective mixing, with relatively rapid vertical dispersion, and high ground-level concentrations.

\section{Conclusions and Recommendations for Further Research}

| Thise study has addressed additional evaluations for low wind conditions involving tall stack releases for which multiple years of concurrent emissions, meteorological data, and monitoring data were available. The modeling cases that were the focus of this study involved applications with only one level of meteorological data and no direct turbulence measurements or vertical temperature gradient observations.

For the North Dakota evaluation, the AERMOD model over-predicted ${ }_{2}$ using the design concentration $\left(99^{\text {th }}\right.$ percentile peak daily 1 hour maximum $)$ as the metric for each monitor. For 
the relatively low elevation monitors, the results were similar for both the default and beta options and are within 5-30\% of the monitored concentrations depending on the model option.

The modeling result for the elevated DGC\#17 monitor showed that this location is sensitive to terrain as the ratio of modeled to monitored concentration is over 2 . However, when this location was modeled with the low wind beta option, the ratio was notably better, at less than 1.3. Furthermore, the low wind speed beta option changed the AERMOD's focus on peak predictions conditions from mostly nighttime to mostly daytime periods, somewhat more in line with observations. Even for a minimum $\underline{\sigma}_{\underline{V}} \operatorname{sigma} \vee$ as high as $0.5 \mathrm{~m} / \mathrm{s}^{-1} \mathrm{~m} / \mathrm{sec}$, all of the AERMOD modeling results were conservative or relatively unbiased (for the design concentration). The North Dakota evaluation results for the sub-hourly (SHARP) modeling were, on average, relatively unbiased, with a predicted-to-observed design concentration ratio ranging from 0.89 to 1.2 . With a $10 \%$ tolerance in the $\mathrm{SO}_{2}$ monitored values, we find that the $\underline{\text { SHARP performance is quite good.this range of results is basically unbiased. Slightly higher }}$ $\underline{\text { SHARP predictions would be expected if AERMOD were run with the LOWWIND1 option }}$ deployed.

For the Gibson flat terrain evaluation, AERMOD with hourly averaged meteorological data overpredicted at three of the four monitors between $30-50 \%$, and about $10 \%$ at the fourth monitor. The AERMOD results did not vary much with the various low wind speed options in this flat terrain setting. AERMOD with sub-hourly meteorological data (SHARP) had the best (least biased predicted-to-observed ratio of design concentrations) performance among the five cases modeled. Over the four monitors, the range of predicted-to-observed ratios for SHARP was a narrow one, ranging from a slight under-prediction by $2 \%$ to an over-prediction by $14 \%$. All other modeling options had a larger range of results.

The overall findings with the low wind speed testing on these tall stack databases indicate that:

- The AERMOD low wind speed options have a minor effect for flat terrain locations.

- The AERMOD low wind speed options have a more significant effect with AERMOD modeling for elevated terrain locations, and the use of the LOWWIND2 option with a minimum $\underline{\sigma}_{\underline{V}}$ sigma- $\vee$ on the order of $0.5 \mathrm{~m} / \mathrm{s}^{-1} \mathrm{~m} / \mathrm{sec}$ is appropriate.

- The AERMOD sub-hourly modeling (SHARP) results are mostly in therelatively unbiased range (modeled to observed design concentration ratios between 0.9 and 1.1) for the two databases tested with that option. 
- The AERMOD low wind speed options improve the consistency of meteorological conditions associated with the highest observed and predicted concentration events.

Further analysis of the low wind speed performance of AERMOD with either the SHARP procedure or the use of the minimum $\sigma_{\mathrm{v}}$ specifications is encouraginged. However, SHARP can only be used if sub-hourly meteorological data is available. -For Automated Surface Observing Stations (ASOS) with 1-minute data, this option is a possibility.

Although the SHARP results reported in this paper are encouraging, further testing is recommended to determine the optimal sub-hourly averaging time (no less than 10 minutes is recommended) and whether other adjustments to AERMOD (e.g., total disabling of the meander option) is recommended. -Another way to implement the sub-hourly information in AERMOD and to avoid the laborious method of running AERMOD several times for SHARP would be to include a distribution, or range, of the sub-hourly wind directions to AERMOD so that the meander calculations could be refined.

For most modeling applications that use hourly averages of meteorological data with no knowledge of the sub-hourly wind distribution, it appears that the best options with the current AERMOD modeling system are to implement the AERMET beta $\mathrm{u}_{2}$ improvements and to use a minimum $\sigma_{\mathrm{v}}$ value on the order of $0.5 \mathrm{~ms}^{-1} \mathrm{~m} / \mathrm{sec} / \mathrm{sec}$.

It is noteworthy that EPA has recently approved (EPARegion 4, 2015) as a site-specific model

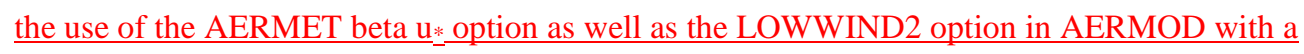
minimum $\sigma_{\underline{v}}$ of $0.4 \mathrm{~ms}^{-1} \mathrm{~m} / \mathrm{sec}$. This model, which was evaluated with site-specific

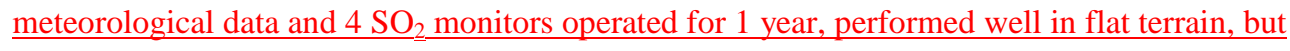
overpredicted in elevated terrain, where a minimum $\sigma_{\mathrm{V}}$ value of $0.6 \mathrm{~ms}^{-1} \mathrm{~m} / \mathrm{sec}$ performed better. This results in an average value of the minimum $\sigma_{\underline{v}}$ of about $0.5 \mathrm{~ms}^{-1} \mathrm{~m} / \mathrm{sec}$, consistent with the findings of Hanna (1990).

The concept of a minimum horizontal wind fluctuation speed of about $0.5 \mathrm{~m} / \mathrm{s}{ }^{-1} \mathrm{~m} / \mathrm{sec}$ is further supported by the existence of vertical changes (shears) in wind direction (as noted by Etling, 1990) that can result in effective horizontal shearing of a plume that is not accounted for in AERMOD. Although we did not test this concept here, the concept of vertical wind shear 
effects, which are more prevalent in de-coupled stable conditions than in well-mixed convective conditions suggests that it would be beneficial to have a "split minimum $\underline{\sigma}_{v}$ sigmaAERMOD that enables the user to specify separate minimum $\sigma_{\mathrm{v}}$ values for stable and unstable conditions. This capability would, of course, be backward-compatible to the current minimum

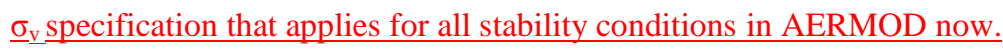

\section{References}

Anfossi, D., D. Oettl, G. Degrazia, A. Goulart. 2005. An analysis of sonic anemometer observations in low wind speed conditions. Boundary Layer Meteorology 114, 179-203.

Barad, M.L., 1958. Project Prairie Grass, a field program in diffusion. Geophys. Res. Pap. 59. Air Force Cambridge Centre.

Brett, A. C., and S. E. Tuller, 1991. The autocorrelation of hourly wind speed observations. Journal of Applied Meteorology 30, 823-833.

Chambers, J. M., W. S. Cleveland, B. Kleiner, and P.A. Tukey. 1983. Comparing Data Distributions. Graphical Methods for Data Analysis. Bell Laboratories. Wadsworth International Group and Duxbury Press.

Chowdhury, B., R. I. Sykes, D. Henn, P. Karamchandani. 2014. SCICHEM Version 3.0 (Beta 2) Technical Documentation. Available at http://sourceforge.net/projects/epridispersion/files/SCICHEM/SCICHEM3.0b2.zip/download.

Cimorelli, A.J., S.G. Perry, A. Venkatram, J.C. Weil, R.J. Paine, R.B. Wilson, R.F. Lee, W.D. Peters, and R.W. Brode. 2005. AERMOD: A Dispersion Model for Industrial Source Applications. Part I: General Model Formulation and Boundary Layer Characterization. Journal of Applied Meteorology 44, 682-693.

Electric Power Research Institute (EPRI). 2013. Sub Hourly AERMOD Run Procedure (SHARP), version 13011 User's Guide. Research Project 1025622. http://sourceforge.net/projects/epri-dispersion/files/SHARP/ (accessed June 10, 2015).

Etling, D. 1990. On Plume Meandering under Stable Stratification. Atmospheric. Environment 8, 1979-1985.

Hanna, S. R. 1990. Lateral Dispersion in Light-Wind Stable Conditions, Nuovo Cimento 13, 889-894.

Irwin, J. S. 2014. A suggested method for dispersion model evaluation. Journal of the Air \& Waste Management Association, 64:3, 255-264. 
$\underline{\text { Kaplan, M. and R.J. Paine. 2012. Comparison of AERMOD Modeled 1-Hour } \mathrm{SO}_{2}}$ Concentrations to Observations at Multiple Monitoring Stations in North Dakota. Presented at the 105th Annual Conference and Exhibition of the Air \& Waste Management Association, San Antonio, TX.

Luhar, A.K. and K.N. Rayner. 2009. Methods to estimate surface fluxes of momentum and heat from routine weather observations for dispersion applications under stable stratification. Boundary Layer Meteorology 132, 437-454.

Mahrt, L. 1998. Stratified Atmospheric Boundary Layers and Breakdown of Models. Theor. Comput. Fluid Dyn. 11, 263-279.

Paine, R., J.A. Connors, and C.D. Szembek, 2010. AERMOD Low Wind Speed Evaluation Study: Results and Implementation. Paper 2010-A-631-AWMA, presented at the 103rd Annual Conference, Air \& Waste Management Association, Calgary, Alberta, Canada.

Paine, R., F. Tringale, and S. Gossett, 2013. Resolution of 1-hour SO2 Non-attainment Area in Kingsport, TN: Advanced Meteorological and Monitoring Study. Presented at the Air \& Waste Management Association's Specialty Conference. Raleigh, NC. March 2013.

Pasquill, F. and F. B. Smith. 1983. Atmospheric Diffusion. Ellis Horwood. Series in Environmental Science.

Perez, I., M. Garcia, M. Sanchez, B. De Torre. 2004. Autocorrelation Analysis of Meteorological Data from a RASS Sodar. Journal of Applied Meteorology, 43: 1213-1223.

Perry, S.G., A.J. Cimorelli, R.J. Paine, R.W. Brode, J.C. Weil, A. Venkatram, R.B. Wilson, R.F. Lee, and W.D. Peters. 2005. AERMOD: A Dispersion Model for Industrial Source Applications. Part II: Model Performance against 17 Field Study Databases. Journal of Applied Meteorology 44, 694-708.

Rayner, K. 2013. Personal communication with Robert Paine, AECOM.

Sagendorf, J. F. and Dickson, C. R. 1974. Diffusion under Low Windspeed, Inversion Conditions. NOAA Technical Memorandum 52, 89 pp.

Smedman, A. S. 1988. Observations of a Multi-Level Turbulence Structure in a Very Stable Atmospheric Boundary Layer. Boundary Layer Meteorology 66, 105-126.

Strimaitis, D., E. Insley, M. Korc, and F. Lurmann.1993. User's Guide for the Model Evaluation Methodology (MEM) System for Comparing Model Performance, Version 1.0. STI93261-1392-FR. Prepared for U.S. Environmental Protection Agency, Research Triangle Park, NC 27711. 
Szembek, C., R. Paine, and S. Gossett. 2013. Resolution of 1-hour SO2 Non-attainment Area in Kingsport, TN: Model Evaluation Analysis Results to Date. Presented at the Air \& Waste Management Association's Specialty Conference. Raleigh, NC. March 2013.

U.S. Environmental Protection Agency. 2003. Revision to the Guideline on Air Quality Models: Adoption of a Preferred Long Range Transport Model and Other Revisions; Final Rule. Federal Register, Volume 68, No. 72, April 15, 2003. http://www.gpo.gov/fdsys/pkg/FR-200304-15/html/03-8542.htm (accessed June 10, 2015).

U.S. Environmental Protection Agency. 2004. AERMOD: Description of Model Formulation. (EPA-454/R-03-004). http://www.epa.gov/ttn/scram/7thconf/aermod/aermod_mfd.pdf. (accessed June 10, 2015).

U.S. Environmental Protection Agency. 2005. Revision to the Guideline on Air Quality Models: Adoption of a Preferred General Purpose (Flat and Complex Terrain) Dispersion Model and Other Revisions. Final Rule. Federal Register, Volume 70, No. 216, November 9, 2005. http://www.gpo.gov/fdsys/pkg/FR-2005-11-09/html/05-21627.htm. (accessed June 10, 2015).

U.S. Environmental Protection Agency. 2009. AERMOD Implementation Guide (AIG). http://www.epa.gov/ttn/scram/7thconf/aermod/aermod_implmtn_guide_19March2009.pdf (accessed June 10, 2015).

U.S. Environmental Protection Agency. 2012. AERMET 12345 Model Change Bulletin MCB\#3. http://www.epa.gov/ttn/scram/7thconf/aermod/aermet_mcb3.txt. (accessed June 10, 2015).

U.S. Environmental Protection Agency. 2013. Quality Assurance Handbook for Air Pollution Measurement Systems, Volume II, Ambient Air Quality Monitoring Program. http://www.epa.gov/ttnamti1/files/ambient/pm25/qa/QA-Handbook-Vol-II.pdf (accessed June 10, 2015).

U.S. Environmental Protection Agency. 2014a. Webinar: AERMOD Modeling System Update, January 15, 2014. http://www.epa.gov/ttn/scram/webinar/AERMOD_13350_Update/AERMOD_System_Update_ Webinar_01-14-2014_FINAL.pdf (accessed June 10, 2015). 
U.S. Environmental Protection Agency. 2014b. Webinar: AERMOD Modeling System
Update, August 14,2014. http://www.epa.gov/ttn/scram/webinar/AERMOD_14134-
NO2_Memo/20140812-Webinar_Slides.pdf (accessed June 10, 2015).
U.S. Environmental Protection Agency. 2015. Approval of Alternative Model Request:
Modeling of Sulfur Dioxide Emissions from Eastman Chemical Company, Sullivan County,
Tennessee, 2010 Sulfur Dioxide National Ambient Air Quality Standards (NAAQS)
Nonattainment Area. Letter to Mr. Barry R. Stephens, Tennessee Department of Environment
and Conservation from Beverly H. Bannister, Director of Air, Pesticides and Toxics
$\underline{\text { Management Division (EPA Region 4). }}$ low-wind-speed inversion conditions. Nuclear. Safety 17, 223-230.

Venkatram, A., R.W. Brode, A.J. Cimorelli, J.T. Lee, R.J. Paine, S.G. Perry, W.D. Peters, J.C. Weil, and R.B. Wilson. 2001. A complex terrain dispersion model for regulatory applications. Atmospheric .Environment. 35, 4211-4221.

Wilson, R. B., Start, G. E., Dickson, C. R., and Ricks, N. R. 1976. Diffusion under low wind speed conditions near Oak Ridge, Tennessee, NOAA Technical Memorandum ERL ARL$\underline{61,83 \mathrm{pp} .}$

Qian, W. and A. Venkatram. 2011. Performance of steady-state dispersion models under low wind-speed conditions. Boundary Layer Meteorology, 138, pp 475-491.

\section{About the Authors}

Robert Paine, CCM, QEP, is an associate vice president and technical director, Olga Samani and Mary Kaplan are senior air quality meteorologists with AECOM's Air Quality Modeling group in Chelmsford, MA.

Eladio Knipping is a principal technical leader in the Environment Sector at the Electric Power Research Institute office in Washington, DC.

Naresh Kumar is a senior program manager of air quality in the environment sector at the Electric Power Research Institute office in Palo Alto, CA. 

Table 1. Databases Selected for the Model Evaluation

\begin{tabular}{|l|c|c|}
\hline & $\begin{array}{c}\text { Mercer County, } \\
\text { North Dakota }\end{array}$ & $\begin{array}{c}\text { Gibson Generating Station, } \\
\text { Indiana }\end{array}$ \\
\hline $\begin{array}{l}\text { Number of emission sources } \\
\text { modeled }\end{array}$ & 15 & 5 \\
\hline Number of $\mathrm{SO}_{2}$ monitors & $\begin{array}{c}5 \\
\text { (one above stack top for } \\
\text { several sources) }\end{array}$ & $\begin{array}{c}4 \\
\text { (all below stack top) }\end{array}$ \\
\hline Type of terrain & Rolling & Flat \\
\hline $\begin{array}{l}\text { Meteorological years and data } \\
\text { source }\end{array}$ & $\begin{array}{c}2007-2010 \\
\text { local 10-meter tower data }\end{array}$ & $\begin{array}{c}\text { 2008-2010 } \\
\text { Evansville airport }\end{array}$ \\
\hline Meteorological data time step & Hourly and sub-hourly & Hourly and sub-hourly \\
\hline $\begin{array}{l}\text { Emissions and } \\
\text { exhaust data }\end{array}$ & $\begin{array}{c}\text { Actual hourly variable } \\
\text { emissions and velocity, } \\
\text { fixed temperature }\end{array}$ & $\begin{array}{c}\text { Actual hourly variable } \\
\text { emissions and velocity, fixed } \\
\text { temperature }\end{array}$ \\
\hline
\end{tabular}


Table 2. Source Information

\begin{tabular}{|c|c|c|c|c|c|c|c|}
\hline $\begin{array}{l}\text { Data } \\
\text { Base }\end{array}$ & $\begin{array}{l}\text { Source } \\
\text { ID }\end{array}$ & UTM X (m) & UTM Y (m) & $\begin{array}{l}\text { Base Elev. } \\
\quad(\mathbf{m})\end{array}$ & $\begin{array}{l}\text { Stack Height } \\
(\mathrm{m})\end{array}$ & $\begin{array}{l}\text { Exit Temp } \\
\quad(\mathbf{K})\end{array}$ & $\begin{array}{c}\text { Stack } \\
\text { Dia. } \\
\text { (m) }\end{array}$ \\
\hline ND & Antelope Valley & 285920 & 5250189 & 588.3 & 182.9 & Vary & 7.0 \\
\hline ND & Antelope Valley & 285924 & 5250293 & 588.3 & 182.9 & Vary & 7.0 \\
\hline ND & Leland Olds & 324461 & 5239045 & 518.3 & 106.7 & Vary & 5.3 \\
\hline ND & Leland Olds & 324557 & 5238972 & 518.3 & 152.4 & Vary & 6.7 \\
\hline ND & Milton R Young & 331870 & 5214952 & 597.4 & 171.9 & Vary & 6.2 \\
\hline ND & Milton R Young & 331833 & 5214891 & 600.5 & 167.6 & Vary & 9.1 \\
\hline ND & Coyote & 286875 & 5233589 & 556.9 & 151.8 & Vary & 6.4 \\
\hline ND & Stanton & 323642 & 5239607 & 518.2 & 77.7 & Vary & 4.6 \\
\hline ND & Coal Creek & 337120 & 5249480 & 602.0 & 201.2 & Vary & 6.7 \\
\hline ND & Coal Creek & 337220 & 5249490 & 602.0 & 201.2 & Vary & 6.7 \\
\hline ND & $\begin{array}{l}\text { Dakota Gasification } \\
\text { Company }\end{array}$ & 285552 & 5249268 & 588.3 & 119.8 & Vary & 7.0 \\
\hline ND & $\begin{array}{l}\text { Dakota Gasification } \\
\text { Company }\end{array}$ & 285648 & 5249553 & 588.3 & 68.6 & Vary & 0.5 \\
\hline ND & $\begin{array}{l}\text { Dakota Gasification } \\
\text { Company }\end{array}$ & 285850 & 5248600 & 588.3 & 76.2 & Vary & 1.0 \\
\hline ND & $\begin{array}{l}\text { Dakota Gasification } \\
\text { Company }\end{array}$ & 285653 & 5249502 & 588.3 & 30.5 & Vary & 0.5 \\
\hline Gibson & Gibson 1 & 432999 & 4247189 & 119.0 & 189.0 & 327.2 & 7.6 \\
\hline Gibson & Gibson 2 & 432999 & 4247189 & 119.0 & 189.0 & 327.2 & 7.6 \\
\hline Gibson & Gibson 3 & 432923 & 4247251 & 118.5 & 189.0 & 327.2 & 7.6 \\
\hline Gibson & Gibson 4 & 432886 & 4247340 & 117.9 & 152.4 & 327.2 & 7.2 \\
\hline Gibson & Gibson 5 & 432831 & 4247423 & 116.3 & 152.4 & 327.2 & 7.2 \\
\hline
\end{tabular}


Table 3. Monitor/Receptor Locations

\begin{tabular}{|l|l|c|c|c|}
\hline $\begin{array}{l}\text { Data } \\
\text { base }\end{array}$ & Monitor & $\begin{array}{c}\text { UTM X } \\
(\mathbf{m})\end{array}$ & $\begin{array}{c}\text { UTM Y } \\
(\mathbf{m})\end{array}$ & $\begin{array}{c}\text { Monitor } \\
\text { Elevation } \\
\text { Above the } \\
\text { Ground (m) }\end{array}$ \\
\hline ND & DGC-\#12 & 291011 & 5244991 & 593.2 \\
\hline ND & DGC-\#14 & 290063 & 5250217 & 604.0 \\
\hline ND & DGC-\#16 & 283924 & 5252004 & 629.1 \\
\hline ND & DGC-\#17 & 279025 & 5253844 & 709.8 \\
\hline ND & Beulah & 290823 & 5242062 & 627.1 \\
\hline Gibson & Mt. Carmel & 432424 & 4250202 & 119.0 \\
\hline Gibson & East Mt. Carmel & 434654 & 4249666 & 119.3 \\
\hline Gibson & Shrodt & 427175 & 4247182 & 138.0 \\
\hline Gibson & Gibson Tower & 434792 & 4246296 & 119.0 \\
\hline${ }^{(a)}$ This monitor's elevation is above stack top for several of the ND sources. \\
\hline
\end{tabular}


Table 4. North Dakota Ratio of Monitored to Modeled Design Concentrations*

\begin{tabular}{|c|c|c|c|c|}
\hline Test Case & Monitor & Observed & Predicted & Ratio \\
\hline \multirow{5}{*}{$\begin{array}{l}\text { Test Case } 1 \\
\text { (Default AERMET, } \\
\text { Default AERMOD) }\end{array}$} & DGC-\#12 & 91.52 & 109.96 & 1.20 \\
\hline & DGC\#14 & 95.00 & 116.84 & 1.23 \\
\hline & DGC-\#16 & 79.58 & 119.94 & 1.51 \\
\hline & DGC\#17 & 83.76 & 184.48 & 2.20 \\
\hline & Beulah & 93.37 & 119.23 & 1.28 \\
\hline \multirow{5}{*}{$\begin{array}{l}\text { Test Case } 2 \\
\text { (Beta AERMET, } \\
\text { Default AERMOD) }\end{array}$} & DGC\#12 & 91.52 & 109.96 & 1.20 \\
\hline & DGC-\#14 & 95.00 & 116.84 & 1.23 \\
\hline & DGC\#16 & 79.58 & 119.94 & 1.51 \\
\hline & DGC\#17 & 83.76 & 127.93 & 1.53 \\
\hline & Beulah & 93.37 & 119.23 & 1.28 \\
\hline \multirow{5}{*}{$\begin{array}{l}\text { Test Case } 3 \\
\text { (Beta AERMET, } \\
\text { AERMOD with } \\
\text { LOWWIND2 } \underline{\sigma}_{\mathrm{V}} \\
\text { Sigma V }=0.3 \mathrm{~m} / \mathrm{s}= \\
{ }^{+} \mathrm{m} / \mathrm{sec} \text { ) }\end{array}$} & DGC-\#12 & 91.52 & 103.14 & 1.13 \\
\hline & DGC\#14 & 95.00 & 110.17 & 1.16 \\
\hline & DGC-\#16 & 79.58 & 111.74 & 1.40 \\
\hline & DGC\#17 & 83.76 & 108.69 & 1.30 \\
\hline & Beulah & 93.37 & 106.05 & 1.14 \\
\hline \multirow{5}{*}{$\begin{array}{l}\text { Test Case } 4 \\
\text { (Beta AERMET, } \\
\text { AERMOD with } \\
\text { LOWWIND } 2 \underline{\sigma}_{\mathrm{V}} \\
\text { Sigma V }=0.5 \mathrm{~m} / \mathrm{s}^{=} \\
{ }^{+} \mathrm{m} / \mathrm{sec} \text { ) }\end{array}$} & DGC\#12 & 91.52 & 95.86 & 1.05 \\
\hline & DGC-\#14 & 95.00 & 100.50 & 1.06 \\
\hline & DGC\#16 & 79.58 & 106.65 & 1.34 \\
\hline & DGC-\#17 & 83.76 & 101.84 & 1.22 \\
\hline & Beulah & 93.37 & 92.32 & 0.99 \\
\hline \multirow{5}{*}{$\begin{array}{l}\text { Test Case } 5 \\
\text { (SHARP) }\end{array}$} & DGC-\#12 & 91.52 & 82.18 & 0.90 \\
\hline & DGC \#14 & 95.00 & 84.24 & 0.89 \\
\hline & DGC-\#16 & 79.58 & 95.47 & 1.20 \\
\hline & DGC\#17 & 83.76 & 88.60 & 1.06 \\
\hline & Beulah & 93.37 & 86.98 & 0.93 \\
\hline
\end{tabular}


Table 5. Gibson Ratio of Monitored to Modeled Design ConcentrationsValues*-

\begin{tabular}{|c|c|c|c|c|}
\hline Test Case & Monitor & Observed & Predicted & Ratio \\
\hline \multirow{4}{*}{$\begin{array}{l}\text { Test Case } 1 \\
\text { (Default AERMET, } \\
\text { Default AERMOD) }\end{array}$} & Mt. Carmel & 197.25 & 278.45 & 1.41 \\
\hline & East Mt. Carmel & 206.89 & 230.74 & 1.12 \\
\hline & Shrodt & 148.16 & 193.71 & 1.31 \\
\hline & Gibson Tower & 127.12 & 189.63 & 1.49 \\
\hline \multirow{4}{*}{$\begin{array}{l}\text { Test Case } 2 \\
\text { (Beta AERMET, } \\
\text { Default AERMOD) }\end{array}$} & Mt. Carmel & 197.25 & 287.16 & 1.46 \\
\hline & East Mt. Carmel & 206.89 & 229.22 & 1.11 \\
\hline & Shrodt & 148.16 & 193.71 & 1.31 \\
\hline & Gibson Tower & 127.12 & 189.63 & 1.49 \\
\hline \multirow{4}{*}{$\begin{array}{l}\text { Test Case } 3 \\
\text { (Beta AERMET, } \\
\text { AERMOD with } \\
\text { LOWWIND2 } \underline{\sigma}_{\underline{v}} \\
\text { Sigma } V=0.3 \mathrm{~m} / \mathrm{s}^{2}\end{array}$} & Mt. Carmel & 197.25 & 280.32 & 1.42 \\
\hline & East Mt. Carmel & 206.89 & 224.65 & 1.09 \\
\hline & Shrodt & 148.16 & 192.22 & 1.30 \\
\hline & Gibson Tower & 127.12 & 184.82 & 1.45 \\
\hline \multirow{4}{*}{$\begin{array}{l}\text { Test Case } 4 \\
\text { (Beta AERMET, } \\
\text { AERMOD with } \\
\text { LOWWIND2 } \underline{\sigma}_{\mathrm{V}} \\
\text { Sigma V }=0.5 \mathrm{~m} / \mathrm{s}\end{array}$} & Mt. Carmel & 197.25 & 277.57 & 1.41 \\
\hline & East Mt. Carmel & 206.89 & 224.65 & 1.09 \\
\hline & Shrodt & 148.16 & 176.81 & 1.19 \\
\hline & Gibson Tower & 127.12 & 192.22 & 1.51 \\
\hline \multirow{4}{*}{$\begin{array}{l}\text { Test Case } 5 \\
\text { (SHARP) }\end{array}$} & Mt. Carmel & 197.25 & 225.05 & 1.14 \\
\hline & East Mt. Carmel & 206.89 & 202.82 & 0.98 \\
\hline & Shrodt & 148.16 & 148.64 & 1.00 \\
\hline & Gibson Tower & 127.12 & 136.41 & 1.07 \\
\hline
\end{tabular}

